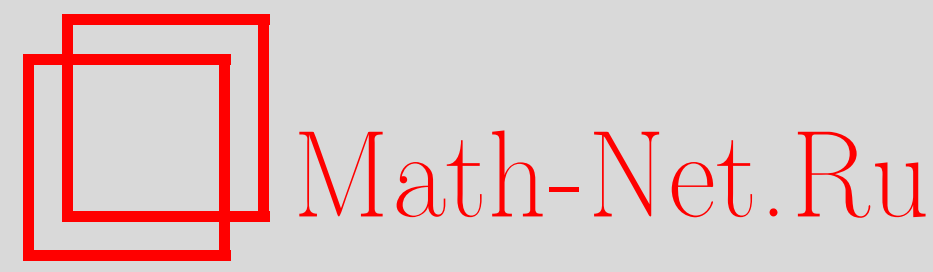

Г. В. Сандраков, Осреднение системы уравнений теории упругости с контрастными коэффициентами, Матем. сб., 1999, том 190, номер 12, 37-92

DOI: https://doi.org/10.4213/sm443

Использование Общероссийского математического портала Math-Net.Ru подразумевает, что вы прочитали и согласны с пользовательским соглашением

http://www . mathnet.ru/rus/agreement

Параметры загрузки:

IP: 3.80 .253 .173

26 апреля 2023 г., 06:41:56 


\title{
Г.В. Сандраков \\ Осреднение системы уравнений теории упругости с контрастными коэффициентами
}

\begin{abstract}
Рассматривается нестационарная задача линеаризованной теории упругости в периодической среде с порами, заполненньми легкодеформируемым материалом. Период рассматриваемой среды задается мальм положительным параметром. Предполагается, что плотность и отношение минимального и максимального модулей упругости легкодеформируемого материала также являются малыми положительньми параметрами. Получены осредненные системы уравнений, решения которых приближают решения рассматриваемой задачи, и доказаны оценки точности соответствующих приближений. Вид осредненных уравнений и оценки точности приближения существенно зависят от геометрических свойств множеств, занимаемых легкодеформируемьм материалом, и от выполнения некоторых предельых соотношений между соответствуюшими малыми параметрами.
\end{abstract}

Библиограффия: 17 названий.

\section{§1. Введение}

В работе рассматривается нестационарная система уравнений линеаризованной теории упругости с коэффициентами, зависящими от двух малых положительных параметров $\varepsilon$ и $\sigma$. Параметр мелкомасштабности $\varepsilon$ задает период коэффициентов этой системы, а величина, обратная параметру контрастности $\sigma$, характеризует разброс коэффициентов тензора упругости. Начально-краевая задача для такой системы уравнений моделирует в линейном приближении упругое поведение микронеоднородной среды (композита) с порами, заполненньми легкодеформируемым материалом и расположенньми периодическим образом с периодом $\varepsilon$. Предполагается, что при $\varepsilon \rightarrow 0$ выполнены соотношение $\sigma \rightarrow 0$ и одно из следуюших трех условий:

$$
\begin{aligned}
& \frac{\sigma}{\varepsilon^{2}} \rightarrow \infty \\
& \frac{\sigma}{\varepsilon^{2}} \rightarrow \vartheta \\
& \frac{\sigma}{\varepsilon^{2}} \rightarrow 0,
\end{aligned}
$$

где $\vartheta$ - заданная положительная постоянная. Будут получены осредненные (предельные) системы уравнений, решения которых приближают решения рассматриваемой системы, и доказаны оценки точности такого приближения.

Работа выполнена при финансовой поддержке Российского фонда фундаментальных исследований (грант № 96-01-01394) и форонда INTAS (грант № 96-1061).

$$
\text { (C) Г.В. САНдРАКОВ } 1999
$$


Пусть для $n \geqslant 2$ заданы ограниченная область $\Omega \subset \mathbb{R}^{n}$ с гладкой (класса $C^{\infty}$ ) границей, положительное $T$, финитные в $\Omega$ и гладкие вектор-функции $f, g_{k} \in C_{0}^{\infty}([0, T] \times \Omega)^{n}$ и $w_{0}, w_{1} \in C_{0}^{\infty}(\Omega)^{n}$, где $k=1, \ldots, n$. Определим вектор-функцию $u=\left(u_{1}, \ldots, u_{n}\right)$ как решение начально-краевой задачи для нестационарной системы уравнений линеаризованной теории упругости:

$$
\begin{gathered}
m_{\varepsilon} \frac{\partial^{2} u}{\partial t^{2}}-\frac{\partial}{\partial x_{h}}\left(A_{\sigma \varepsilon}^{h k} \frac{\partial u}{\partial x_{k}}\right)=r_{\varepsilon} f+\frac{\partial}{\partial x_{h}}\left(B_{\sigma \varepsilon}^{h k} g_{k}\right) \quad \text { в } \Omega \times(0, T), \\
\left.u\right|_{t=0}=w_{0} \text { в } \Omega,\left.\quad u^{\prime}\right|_{t=0}=w_{1} \text { в } \Omega, \quad u=0 \text { на } \partial \Omega \times(0, T),
\end{gathered}
$$

зависящей от малых положительных параметров $\varepsilon$ и $\sigma$, следующим образом.

Пусть $F_{1}$ и $F_{2}$ являются открытыми 1 -периодическими подмножествами $\mathbb{R}^{n}$ с границами класса $C^{2}, \bar{F}_{1} \cap \bar{F}_{2}=\varnothing, F_{0} \equiv \mathbb{R}^{n} \backslash\left(\bar{F}_{1} \cup \bar{F}_{2}\right)$ является множеством с границей класса $C^{2}$ и $F_{s}^{\varepsilon} \equiv \varepsilon F_{s}=\left\{\varepsilon x: x=\left(x_{1}, \ldots, x_{n}\right) \in F_{s}\right\}$ для $s=0,1,2$. Здесь и далее 1-периодичность множества (функции) означает периодичность характеристической функции этого множества (этой функции) с периодом 1 по каждому $x_{k}$, $k=1, \ldots, n$. Таким образом, $Y=[0,1]^{n}$ является ячейкой периодичности, и множества $F_{0}, F_{1}, F_{2}$ вполне определяются множествами $Y_{0}=Y \cap F_{0}, Y_{1}=Y \cap F_{1}$ и $Y_{2}=Y \cap F_{2}$. Предполагается, что множества $Y_{1}$ и $Y_{2}$ связны и выполнены условия положительности для мер Лебега в $\mathbb{R}^{n}$ :

$$
\theta_{0}=\operatorname{mes}\left(Y_{0}\right)>0, \quad \theta_{1}=\operatorname{mes}\left(Y_{1}\right)>0, \quad \theta_{2}=\operatorname{mes}\left(Y_{2}\right)>0 .
$$

Кроме того, отождествляя противоположные грани $Y$, множества $Y_{0}, Y_{1}$ и $Y_{2}$ можно также рассматривать как подмногообразия тора с общими границами (класca $\left.C^{2}\right): S_{0} \equiv \partial Y_{0}, S_{1} \equiv \partial Y_{1}=\partial Y_{0} \backslash \partial Y_{2}, S_{2} \equiv \partial Y_{2}=\partial Y_{0} \backslash \partial Y_{1}$.

Для $h, k=1, \ldots, n$ матричнозначные функции $A_{\sigma \varepsilon}^{h k}$ и $B_{\sigma \varepsilon}^{h k}$ в (1.4) зависят от малых параметров $\varepsilon$ и $\sigma$ :

$$
\begin{array}{ll}
A_{\sigma \varepsilon}^{h k}=\sigma A_{0}^{h k}\left(\frac{x}{\varepsilon}\right), & B_{\sigma \varepsilon}^{h k}=\sigma B_{0}^{h k}\left(\frac{x}{\varepsilon}\right) \quad \text { в } \quad \Omega_{0}^{\varepsilon} \equiv \Omega \cap F_{0}^{\varepsilon}, \\
A_{\sigma \varepsilon}^{h k}=A_{1}^{h k}\left(\frac{x}{\varepsilon}\right), & B_{\sigma \varepsilon}^{h k}=B_{1}^{h k}\left(\frac{x}{\varepsilon}\right) \quad \text { в } \quad \Omega_{1}^{\varepsilon} \equiv \Omega \cap F_{1}^{\varepsilon}, \\
A_{\sigma \varepsilon}^{h k}=A_{2}^{h k}\left(\frac{x}{\varepsilon}\right), & B_{\sigma \varepsilon}^{h k}=B_{2}^{h k}\left(\frac{x}{\varepsilon}\right) \quad \text { в } \quad \Omega_{2}^{\varepsilon} \equiv \Omega \cap F_{2}^{\varepsilon} .
\end{array}
$$

Предполагается, что для $s=0,1,2$ и $i, j, h, k=1, \ldots, n$ компоненты $a_{i j s}^{h k}(y), b_{i j s}^{h k}(y)$ матричнозначных функций $A_{s}^{h k}(y)$ и $B_{s}^{h k}(y)$ являются 1-периодическими ограниченными (почти всюду) функциями на $F_{s}$ соответственно. Кроме того, для этих компонент выполнены условия симметрии, а для $a_{i j s}^{h k}(y)$ и условие равномерной эллиптичности:

$$
\begin{gathered}
a_{i j s}^{h k}(y)=a_{j i s}^{k h}(y)=a_{h j s}^{i k}(y), \quad b_{i j s}^{h k}(y)=b_{j i s}^{k h}(y)=b_{h j s}^{i k}(y), \\
\alpha \xi_{i h} \xi_{i h} \leqslant a_{i j s}^{h k}(y) \xi_{i h} \xi_{j k} \leqslant \beta \xi_{i h} \xi_{i h} \quad \text { для } y \in Y_{s} \quad \text { и } s=0,1,2,
\end{gathered}
$$

где $\alpha$ и $\beta$ - заданные положительные постоянные и $\xi_{i j}=\xi_{j i}$ для $i, j=1, \ldots, n$. Здесь и далее приняты обычные для теории упругости обозначения и соглашение о суммировании по повторяющимся индексам $i, j, h, k$ от 1 до $n$. Функции $m_{\varepsilon}$ и $r_{\varepsilon}$ в (1.4) зависят только от $\varepsilon$ :

$$
m_{\varepsilon}=m_{s}\left(\frac{x}{\varepsilon}\right), \quad r_{\varepsilon}=r_{s}\left(\frac{x}{\varepsilon}\right) \quad \text { в } \Omega_{s}^{\varepsilon},
$$


где $m_{s}(y)$ и $r_{s}(y)$ являются 1-периодическими ограниченными функциями на $F_{s}$ для $s=0,1,2$. Кроме того, $m_{s}(y)$ отделены от нуля:

$$
\alpha \leqslant m_{s}(y) \leqslant \beta \text { для } y \in Y_{s} \quad \text { и } s=0,1,2 .
$$

Предполагается также, что множество $F_{1}$ является связньм, а множество $F_{2}$ вполне несвязно. Последнее по определению означает, что $\left(\bar{F}_{2}+z\right) \cap \partial Y=\varnothing$ для некоторого $z \in Y$. Это определение введено В. В. Жиковьм [1] в более общей ситуации для фрактальных множеств. Таким образом, между множествами $F_{1}$ и $F_{2}$ имеется существенное различие. Множество $F_{2}$ составлено из изолированных компонент, и нагрузка, приложенная к части этих компонент, не передается на другие компоненты, а для множества $F_{1}$ это не так, поскольку $F_{1}$ связно. Аналогичное замечание относится и к множествам $\Omega_{1}^{\varepsilon}$ и $\Omega_{2}^{\varepsilon}$, что в пределе при $\varepsilon \rightarrow 0$ и $\sigma \rightarrow 0$ приводит к нулевому вкладу компонент $\Omega_{2}^{\varepsilon}$ в осредненный тензор упругости.

В качестве типичного примера двумерных $(n=2)$ множеств $F_{0}, F_{1}$ и $F_{2}$, удовлетворяющих сформулированным условиям, можно выбрать 1-периодическое продолжение множеств $Y_{0}, Y_{1}$ и $Y_{2}$, изображенных на рис. 1 . Поскольку связность множества $Y_{0}$ не предполагается, 1-периодическое продолжение ячейки, изображенной на рис. 2 , также приводит к множествам $F_{0}, F_{1}$ и $F_{2}$, удовлетворяющим сформулированным условиям. В трехмерном случае также несложно привести “типичные" примеры подходящих множеств $F_{0}, F_{1}$ и $F_{2}$. Кроме того, полученные в данной работе утверждения выполнены и в случае, когда $Y_{1}=\varnothing$ или $Y_{2}=\varnothing$ (в этих утверждениях необходимо только обнулить соответствующие функции и постоянные). В этом частном случае система уравнений (1.4) описывает динамическое поведение композита с легкодеформируемыми включениями (при $\left.Y_{2}=\varnothing\right)$ или композита с включениями в легкодеформируемой матрице (при $\left.Y_{1}=\varnothing\right)$.

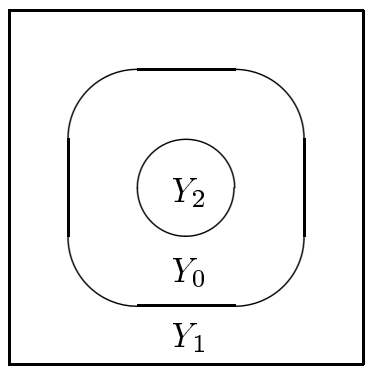

Рис. 1

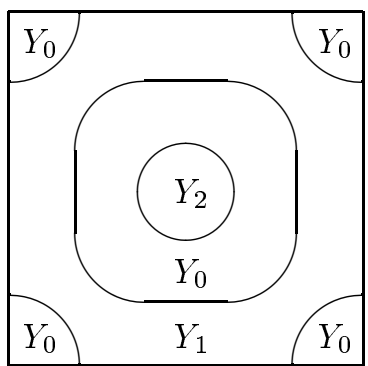

Рис. 2

В рассматриваемом здесь общем случае быстроосциллирующие коэффициенты первой системы уравнений в (1.4) имеют единичный порядок на множестве $\Omega_{12}^{\varepsilon} \equiv \Omega_{1}^{\varepsilon} \cup \Omega_{2}^{\varepsilon}$, но коэффициенты тензора упругости асимптотически малы на $\Omega_{0}^{\varepsilon}$. Кроме того, малость этих коэффициентов контролируется одним из предположений (1.1)-(1.3), а на множества $\Omega_{0}^{\varepsilon}, \Omega_{1}^{\varepsilon}$ и $\Omega_{2}^{\varepsilon}$ наложены условия непустоты $(1.5)$ и некоторой регулярности "внутренних" границ, разделяющих эти множества. Таким образом, система уравнений (1.4) описывает динамическое поведение композита с достаточно сложной структурой. Цель исследования этой системы при сделанных предположениях - подчеркнуть существенное различие вклада компонент $\Omega_{1}^{\varepsilon}$ и $\Omega_{2}^{\varepsilon}$ 
в осредненные задачи и отметить возможную взаимосвязь этих компонент через коэффициенты осредненных задач.

Поскольку коэффициенты в (1.4) могут быть разрывными, следуя [2], определим решение этой задачи как элемент $u \in L^{\infty}\left(0, T ; H_{0}^{1}(\Omega)^{n}\right)$, удовлетворяющий для каждого $\varphi \in H_{0}^{1}(\Omega)^{n}$ и почти всех $t \in(0, T)$ интегральному тождеству

$\int_{\Omega}\left(m_{\varepsilon} \frac{\partial^{2} u}{\partial t^{2}}, \varphi\right) d x+\int_{\Omega}\left(A_{\sigma \varepsilon}^{h k} \frac{\partial u}{\partial x_{k}}, \frac{\partial \varphi}{\partial x_{h}}\right) d x=\int_{\Omega}\left(r_{\varepsilon} f, \varphi\right) d x-\int_{\Omega}\left(B_{\sigma \varepsilon}^{h k} g_{k}, \frac{\partial \varphi}{\partial x_{h}}\right) d x$

и начальным условиям $\left.u\right|_{t=0}=w_{0}$ в $H_{0}^{1}(\Omega)^{n},\left.u_{t}^{\prime}\right|_{t=0}=w_{1}$ в $L^{2}(\Omega)^{n}$. Здесь и далее $(\cdot, \cdot)$ обозначает скалярное произведение векторов из $\mathbb{R}^{n}$ и используются функциональные пространства, определенные, например, в [2]. Известно [2], [3], что сушествует единственное (для фиксированных $\varepsilon$ и $\sigma$ ) решение этой задачи, такое, что $u \in C^{0}\left([0, T] ; H_{0}^{1}(\Omega)^{n}\right)$ и $u_{t}^{\prime} \in C^{0}\left([0, T] ; L^{2}(\Omega)^{n}\right)$.

Основные результаты работы составляют теоремы о предельном поведении решений задачи (1.4) при $\varepsilon \rightarrow 0$ в предположении, что выполнены соотношение $\sigma \rightarrow 0$ и одно из условий (1.1)-(1.3). Для точной формулировки этих утверждений понадобятся некоторые дополнительные обозначения.

Пусть для $p=1, \ldots, n$ матричнозначные функции $N_{1 a}^{p}(y)$ являются 1-периодическими решениями задач Неймана на $Y_{1}$ :

$$
-\frac{\partial}{\partial y_{h}}\left(A_{1}^{h k} \frac{\partial}{\partial y_{k}} N_{1 a}^{p}\right)=\frac{\partial}{\partial y_{h}} A_{1}^{h p} \text { в } Y_{1}, \quad-\nu_{h}\left(A_{1}^{h k} \frac{\partial}{\partial y_{k}} N_{1 a}^{p}\right)=\nu_{h} A_{1}^{h p} \text { на } S_{1},
$$

где $\nu=\left(\nu_{1}, \ldots, \nu_{n}\right)$ - внешняя нормаль к границе $S_{1}$ и использованы обозначения (1.6). Определим для $q, p=1, \ldots, n$ матрицы $\widehat{A}_{1}^{q p}$ с постоянными компонентами равенствами

$$
\widehat{A}_{1}^{q p}=\left\langle A_{1}^{q p}+A_{1}^{q i} \frac{\partial}{\partial y_{i}} N_{1 a}^{p}\right\rangle_{\mathbf{1}} \equiv \int_{Y_{1}}\left(A_{1}^{q p}+A_{1}^{q i} \frac{\partial}{\partial y_{i}} N_{1 a}^{p}\right) d y .
$$

Известно [4], [5], что компоненты матрищ $\widehat{A}_{1}^{q p}$ удовлетворяют условиям симметрии и равномерной эллиптичности (1.7).

Пусть для $p=1, \ldots, n$ матричнозначные функции $N_{1 b}^{p}(y)$ и $N_{2 b}^{p}(y)$ являются 1-периодическими решениями задач Неймана на $Y_{1}$ и $Y_{2}$ :

$$
-\frac{\partial}{\partial y_{h}}\left(A_{s}^{h k} \frac{\partial}{\partial y_{k}} N_{s b}^{p}\right)=\frac{\partial}{\partial y_{h}} B_{s}^{h p} \text { в } Y_{s}, \quad-\nu_{h}\left(A_{s}^{h k} \frac{\partial}{\partial y_{k}} N_{s b}^{p}\right)=\nu_{h} B_{s}^{h p} \text { на } S_{s},
$$

где $\nu=\left(\nu_{1}, \ldots, \nu_{n}\right)$ обозначает также внешнюю нормаль к границе $S_{2}, s=1,2$, и использованы обозначения (1.6). Далее, учитывая (1.6) и (1.8), введем для $q, p=1, \ldots, n$ обозначения

$$
\begin{gathered}
\widehat{B}_{2}^{q p}=\left\langle B_{2}^{q p}+A_{2}^{q i} \frac{\partial}{\partial y_{i}} N_{2 b}^{p}\right\rangle_{\mathbf{2}} \equiv \int_{Y_{2}}\left(B_{2}^{q p}+A_{2}^{q i} \frac{\partial}{\partial y_{i}} N_{2 b}^{p}\right) d y \\
M=\left\langle m_{0}\right\rangle_{\mathbf{o}}+\left\langle m_{1}\right\rangle_{\mathbf{1}}+\left\langle m_{2}\right\rangle_{\mathbf{2}} \equiv \int_{Y_{0}} m_{0} d y+\int_{Y_{1}} m_{1} d y+\int_{Y_{2}} m_{2} d y, \\
\widehat{B}_{1}^{q p}=\left\langle B_{1}^{q p}+A_{1}^{q i} \frac{\partial}{\partial y_{i}} N_{1 b}^{p}\right\rangle_{\mathbf{1}}, \quad R=\left\langle r_{0}\right\rangle_{\mathbf{o}}+\left\langle r_{1}\right\rangle_{\mathbf{1}}+\left\langle r_{2}\right\rangle_{\mathbf{2}} .
\end{gathered}
$$


Определим вектор-функцию $v=\left(v_{1}, \ldots, v_{n}\right)$ как решение начально-краевой задачи для осредненной системы уравнений с постоянными коэффициентами:

$$
\begin{gathered}
M \frac{\partial^{2} v}{\partial t^{2}}-\frac{\partial}{\partial x_{h}}\left(\widehat{A}_{1}^{h k} \frac{\partial v}{\partial x_{k}}\right)=R f+\frac{\partial}{\partial x_{h}}\left(\left(\widehat{B}_{1}^{h k}+\widehat{B}_{2}^{h k}\right) g_{k}\right) \text { в } \Omega \times(0, T), \\
\left.v\right|_{t=0}=w_{0} \text { в } \Omega,\left.\quad v_{t}^{\prime}\right|_{t=0}=w_{1} \text { в } \Omega, \quad v=0 \text { на } \partial \Omega \times(0, T),
\end{gathered}
$$

где использованы обозначения (1.12) и (1.14).

ТЕОРема 1. Пусть выполнено условие (1.1). Предположим, что и является решением задачи (1.4) и $v$-решение задачи (1.15). Тогда

$$
\max _{0 \leqslant t \leqslant T}\|u-v\|_{L^{2}(\Omega)^{n}}^{2} \leqslant C\left(\varepsilon+\sigma+\varepsilon^{2} / \sigma\right)
$$

әде постоянная $C$ не зависит от $\varepsilon$ и $\sigma$.

Пусть матричнозначная функция $E_{1}(y)$ является 1-периодическим решением задачи Дирихле на $Y_{0}$ :

$$
\frac{\partial}{\partial y_{h}}\left(A_{0}^{h k} \frac{\partial}{\partial y_{k}} E_{1}\right)=0 \text { в } Y_{0}, \quad E_{1}=E \text { на } \bar{Y}_{1}, \quad E_{1}=0 \text { на } \bar{Y}_{2},
$$

где $E$ - единичная матрица для $\mathbb{R}^{n}$ и использованы обозначения (1.6). Более традиционно, например, условие $E_{1}=E$ на $\bar{Y}_{1}$ означает, что $E_{1}=E$ на $S_{1}$ и $E_{1}$ продолжена как единичная матрица на $Y_{1}$. Отметим также, что $Y_{0}$ может иметь несколько связных компонент (рис. 2). В этом случае задача (1.16) "распадается" на несколько задач, но на компонентах $Y_{0}$, полностью содержашихся в $Y_{1}$ или $Y_{2}$, решение этих задач совпадает с единичной или нулевой матрищей соответственно.

Используемые до сих пор матрицы (и матричнозначные функции) имели размерность $n \times n$, но в дальнейшем понадобятся и более общие матрицы, размерность которых определяется явно или ясна из контекста. Пусть $\widetilde{n}=n+n(n-1) / 2$ и $E_{2}=\left(e_{1}, \ldots, e_{\tilde{n}}\right)$ обозначает $(n \times \widetilde{n})$-матрицу, столбцы которой образуют базис в линейном пространстве жестких перемешений в $\mathbb{R}^{n}$ (см., например, [3], [5]). Без ограничения общности можно считать, что первые $n$ столбцов $E_{2}$ образуют единичную матрицу, $E_{2}(y)$ определена и непрерывна на $Y_{2}$ и выполнено условие нормировки:

$$
\left\langle m_{2} E_{2}^{T} E_{2}\right\rangle_{2}=\left\langle m_{2}\right\rangle_{2} \widetilde{E},
$$

где $\widetilde{E}$ - единичная матрица для $\mathbb{R}^{\tilde{n}}$ и использованы обозначения (1.8). Здесь $E_{2}^{T}$ обозначает матрицу, сопряженную к $E_{2}$. Доопределим $E_{2}(y)$ на $Y \backslash \bar{Y}_{2}$ как 1-периодическое решение задачи Дирихле

$$
\frac{\partial}{\partial y_{h}}\left(A_{0}^{h k} \frac{\partial}{\partial y_{k}} E_{2}\right)=0 \text { в } Y_{0}, \quad E_{2}=0 \text { на } \bar{Y}_{1}, \quad E_{2}=E_{2} \text { на } \bar{Y}_{2} .
$$


Пусть $(n \times n)$-матричнозначные функции $P(t, y), Q_{1}(t, y)$ и $(n \times \tilde{n})$-матричнозначная функция $Q_{2}(t, y)$ являются 1-периодическими решениями начально-краевых задач на $Y_{0}$ :

$$
\begin{gathered}
m_{0} \frac{\partial^{2} P}{\partial t^{2}}-\vartheta \frac{\partial}{\partial y_{h}}\left(A_{0}^{h k} \frac{\partial P}{\partial y_{k}}\right)=0 \text { в } Y_{0} \times(0, \infty), \\
\left.P\right|_{t=0}=0 \text { в } Y_{0},\left.\quad P_{t}^{\prime}\right|_{t=0}=\frac{r_{0}}{m_{0}} E \text { в } Y_{0}, \quad P=0 \text { на } \bar{Y}_{12} \times(0, \infty), \\
m_{0} \frac{\partial^{2} Q_{s}}{\partial t^{2}}-\vartheta \frac{\partial}{\partial y_{h}}\left(A_{0}^{h k} \frac{\partial Q_{s}}{\partial y_{k}}\right)=0 \text { в } Y_{0} \times(0, \infty), \\
\left.Q_{s}\right|_{t=0}=0 \text { в } Y_{0},\left.\quad Q_{s t}^{\prime}\right|_{t=0}=E_{s} \text { в } Y_{0}, \quad Q_{s}=0 \text { на } \bar{Y}_{12} \times(0, \infty),
\end{gathered}
$$

где $s=1,2, Y_{12}=Y_{1} \cup Y_{2}$ и учтены обозначения (1.2), (1.6) и (1.8). Далее, введем для $s, l=1,2$ и $q, p=1, \ldots, n$ обозначения

$$
\begin{aligned}
& M_{s}=\left\langle m_{s}\right\rangle_{\mathrm{s}}, \quad R_{s}^{0}(t)=\left\langle m_{0} E_{s}^{T} P_{t t}^{\prime \prime}\right\rangle_{\mathbf{o}}, \quad M_{s}^{l}(t)=\left\langle m_{0} E_{l}^{T}\left(Q_{s}\right)_{t t}^{\prime \prime}\right\rangle_{\mathbf{o}}, \\
& a_{s}^{l}=\vartheta\left\langle\frac{\partial E_{l}^{T}}{\partial y_{h}} A_{0}^{h k} \frac{\partial E_{s}}{\partial y_{k}}\right\rangle_{\mathbf{o}}, \quad \widetilde{B}_{2}^{q p}=\left\langle E_{2}^{T}\left(B_{2}^{q p}+A_{2}^{q i} \frac{\partial}{\partial y_{i}} N_{2 b}^{p}\right)\right\rangle_{\mathbf{2}}, \\
& R_{s}=\left\langle E_{s}^{T} r_{s}\right\rangle_{\mathrm{s}}, \quad E_{l \varepsilon}=E_{l}\left(\frac{x}{\varepsilon}\right), \quad Q_{l \varepsilon}=Q_{l}\left(t, \frac{x}{\varepsilon}\right), \quad P_{\varepsilon}=P\left(t, \frac{x}{\varepsilon}\right),
\end{aligned}
$$

где, например, $P(t, y)$ продолжена как 1 -периодическая функция на $\mathbb{R}^{n}$ и тогда $P_{\varepsilon}$ обозначает ограничение этого продолжения при $y=x / \varepsilon$ на $\Omega$. Пусть $\widetilde{w}_{0}, \widetilde{w}_{1}$ обозначают $\widetilde{n}$-мерные вектор-функции, первые $n$ компонент которых равны $w_{0}, w_{1}$ соответственно, а остальные компоненты являются нулевыми. Определим $n$-мерную вектор-функцию $v_{1}$ и $\widetilde{n}$-мерную вектор-функцию $v_{2}$ как решения начально-краевой задачи для осредненной системы уравнений:

$$
\begin{gathered}
M_{1} \frac{\partial^{2} v_{1}}{\partial t^{2}}-M_{1}^{1} *\left(\frac{\partial^{2} v_{1}}{\partial t^{2}}\right)-M_{2}^{1} *\left(\frac{\partial^{2} v_{2}}{\partial t^{2}}\right)+a_{1}^{1} v_{1}+a_{2}^{1} v_{2} \\
-\frac{\partial}{\partial x_{h}}\left(\widehat{A}_{1}^{h k} \frac{\partial v_{1}}{\partial x_{k}}\right)=R_{1} f-R_{1}^{0} * f+\frac{\partial}{\partial x_{h}}\left(\widehat{B}_{1}^{h k} g_{k}\right) \text { в } \Omega \times(0, T), \\
M_{2} \frac{\partial^{2} v_{2}}{\partial t^{2}}-M_{1}^{2} *\left(\frac{\partial^{2} v_{1}}{\partial t^{2}}\right)-M_{2}^{2} *\left(\frac{\partial^{2} v_{2}}{\partial t^{2}}\right)+a_{1}^{2} v_{1}+a_{2}^{2} v_{2} \\
=R_{2} f-R_{2}^{0} * f+\frac{\partial}{\partial x_{h}}\left(\widetilde{B}_{2}^{h k} g_{k}\right) \text { в } \Omega \times(0, T), \\
\left.v_{1}\right|_{t=0}=w_{0} \text { в } \Omega,\left.\quad\left(v_{1}\right)_{t}^{\prime}\right|_{t=0}=w_{1} \text { в } \Omega, \quad v_{1}=0 \text { на } \partial \Omega \times(0, T), \\
\left.v_{2}\right|_{t=0}=\widetilde{w}_{0} \text { в } \Omega,\left.\quad\left(v_{2}\right)_{t}^{\prime}\right|_{t=0}=\widetilde{w}_{1} \text { в } \Omega,
\end{gathered}
$$

где $*$ обозначает свертку по $t$ и использованы обозначения (1.14) и (1.20).

Tеорема 2. Пусть выполнено условие $(1.2), f, g_{k} \in C_{0}^{\infty}((0, T) \times \Omega)^{n}, w_{0}=0$ u $w_{1}=0$, где $k=1, \ldots, n$. Предположим, что и является решением задачи (1.4) и $\left(v_{1}, v_{2}\right)$ - решение задачи (1.21). Тогда

$$
\begin{aligned}
& \max _{0 \leqslant t \leqslant T}\left\|u-E_{1 \varepsilon} v_{1}-E_{2 \varepsilon} v_{2}-P_{\varepsilon} * f+Q_{1 \varepsilon} *\left(\left(v_{1}\right)_{t t}^{\prime \prime}\right)+Q_{2 \varepsilon} *\left(\left(v_{2}\right)_{t t}^{\prime \prime}\right)\right\|_{L^{2}(\Omega)^{n}}^{2} \\
& \quad \leqslant c\left(\varepsilon(1+\vartheta+\sqrt{\vartheta})+\sigma+\varepsilon \frac{1+\sqrt{\vartheta}}{\sqrt{\sigma \vartheta}}\left|\vartheta-\frac{\sigma}{\varepsilon^{2}}\right|\right) \leqslant C\left(\varepsilon+\sigma+\left|\vartheta-\frac{\sigma}{\varepsilon^{2}}\right|\right)
\end{aligned}
$$

әде постоянные $C$ и с не зависят от $\varepsilon, \sigma$ и, кроме того, с не зависит от $\vartheta$. 
Пусть $\chi_{0}^{\varepsilon}, \chi_{1}^{\varepsilon}$ и $\chi_{2}^{\varepsilon}$ обозначают характеристические функции множеств $\Omega_{0}^{\varepsilon}, \Omega_{1}^{\varepsilon}$ и $\Omega_{2}^{\varepsilon}$ соответственно. Определим $n$-мерную вектор-функцию $v_{1}$ и $\tilde{n}$-мерную вектор-функцию $v_{2}$ как решения начально-краевых задач для осредненных систем уравнений с постоянными коэффициентами:

$$
\begin{gathered}
M_{1} \frac{\partial^{2} v_{1}}{\partial t^{2}}-\frac{\partial}{\partial x_{h}}\left(\widehat{A}_{1}^{h k} \frac{\partial v_{1}}{\partial x_{k}}\right)=R_{1} f+\frac{\partial}{\partial x_{h}}\left(\widehat{B}_{1}^{h k} g_{k}\right) \text { в } \Omega \times(0, T), \\
\left.v_{1}\right|_{t=0}=w_{0} \text { в } \Omega,\left.\quad\left(v_{1}\right)_{t}^{\prime}\right|_{t=0}=w_{1} \text { в } \Omega, \quad v_{1}=0 \text { на } \partial \Omega \times(0, T), \\
M_{2} \frac{\partial^{2} v_{2}}{\partial t^{2}}=R_{2} f+\frac{\partial}{\partial x_{h}}\left(\widetilde{B}_{2}^{h k} g_{k}\right) \text { в } \Omega \times(0, T) \\
\left.v_{2}\right|_{t=0}=\widetilde{w}_{0} \text { в } \Omega,\left.\quad\left(v_{2}\right)_{t}^{\prime}\right|_{t=0}=\widetilde{w}_{1} \text { в } \Omega
\end{gathered}
$$

где использованы обозначения (1.12), (1.14) и (1.20).

Теорема 3. Пусть выполнено условие (1.3) и $\nabla_{y}\left(r_{0} / m_{0}\right) \in L^{\infty}\left(Y_{0}\right)^{n}$. Предположим, что и является решением задачи (1.4) и $v_{1}, v_{2}$ - решение задач (1.22). Тогда

$$
\begin{gathered}
\max _{0 \leqslant t \leqslant T}\left\|u-\chi_{1}^{\varepsilon} v_{1}-\chi_{2}^{\varepsilon} E_{2 \varepsilon} v_{2}-\chi_{0}^{\varepsilon} w_{0}-\chi_{0}^{\varepsilon} w_{1} t-\chi_{0}^{\varepsilon}\left(\frac{r_{0}}{m_{0}}\right) \int_{\varepsilon}^{t} \int_{0}^{t} f d t d t\right\|_{L^{2}(\Omega)^{n}}^{2} \\
\leqslant C\left(\varepsilon+\sigma+\frac{\sqrt[4]{\sigma}}{\sqrt{\varepsilon}}\right)
\end{gathered}
$$

где интеграль обозначают вторую первообразную по $t$ и постоянная $C$ не зависит от $\varepsilon u \sigma$.

ЗАмЕчАнИЕ 1 . Проверяется, что в условиях теорем 1-3 достаточно гладкие решения осредненных задач (1.15), (1.21), (1.22) сушествуют и определены однозначно. Гладкость и финитность $f, g_{k}, w_{0}, w_{1}$ (и равенства $w_{0}=0, w_{1}=0$ из условий теоремы 2) используются в доказательстве именно этого утверждения о решениях осредненных задач. Это утверждение для задач (1.15) и (1.22) хорошо известно [3], [6], поскольку при сделанных предположениях на исходные данные вьполнены условия согласования произвольного порядка. Это утверждение для задачи (1.21) доказывается в $§ 2$ методами работы [7], в которой была доказана разрешимость аналогичной задачи для линеаризованной системы уравнений гидродинамики.

ЗАмЕчАниЕ 2. Для построения начальных членов асимптотического разложения решения задачи (1.4) используются методы работ [7] и [8]. Как обычно [4], [7], [8], это построение заключается в последовательном разрешении некоторых периодических задач на ячейке (типа задач $(1.11),(1.13))$, условия разрешимости которых приводят к осредненным задачам (1.15), (1.21) или (1.22) в зависимости от выполнения одного из условий (1.1)-(1.3). Кратко это построение излагается в $\S 3$. После того как начальные члены асимптотики построены, доказательство оценок теорем 1-3 проводится в $\S \S 4-10$ энергетическими методами. При этом учитывается гладкость решений осредненных задач (на самом деле конечного порядка, см. $\S 2$ и и некоторая регулярность решений задач на ячейке, в доказательстве которой используется гладкость (класса $C^{2}$ ) границ $S_{1}$ и $S_{2}$. В доказательстве теоремы 3 используется также условие регулярности отношения коэффициентов 
$r_{0}$ и $m_{0}$. В задачах, встречающихся на практике, коэффициенты $m_{0}, m_{1}, m_{2}, \ldots$, как правило, постоянны, так что это условие не слишком существенно. Отметим также, что с помощью подходящих аппроксимаций исходных данных из теорем 1-3 несложно вывести утверждения о сходимости решений задачи (1.4) при более слабых предположениях на $f, g_{k}, w_{0}$ и $w_{1}$, где $k=1, \ldots, n$.

ЗАмечаниЕ 3 . Теоремы 1-3 выполнены также, когда $Y_{1}=\varnothing$ или $Y_{2}=\varnothing$. Так, например, в последнем случае интегралы по $Y_{2}$ следует считать нулевыми, что согласуется с определением интеграла Лебега. Кроме того, следует выбрать $E_{1}=E, E_{2}=0$ и поэтому $Q_{2}=0, v_{2}=0$, впрочем, это подразумевается определениями решений соответствующих задач. Более того, после соответствующих изменений в формулировках теоремы 1-3 выполнены и в случае, когда имеются $s_{1}$ связных непустых открытых 1 -периодических множеств $F_{1}, \ldots, F_{s_{1}}$ и $s_{2}$ вполне несвязных непустых открытых 1-периодических множеств $F_{s_{1}+1}, \ldots, F_{s_{1}+s_{2}}$ с границами класса $C^{2}$, замыкания которых не пересекаются, и $F_{0} \equiv \mathbb{R}^{n} \backslash\left(\bar{F}_{1} \cup \cdots \cup \bar{F}_{s_{1}+s_{2}}\right)$. Для простоты формулировок и ясности изложения здесь подробно рассматривается случай $s_{1}=1$ и $s_{2}=1$. Относительно общего случая произвольных неотрицательных $s_{1}$ и $s_{2}$ (причем $s_{1}+s_{2}>0$ ) в дальнейшем будут приводиться замечания, достаточные для восстановления соответствующих деталей. Когда выполнено условие (1.1), наиболее сушественное изменение в (1.15) - это замена матриц $\widehat{A}_{1}^{h k}$ и $\widehat{B}_{1}^{h k}+\widehat{B}_{2}^{h k}$ на суммы матриц $\widehat{A}_{1}^{h k}+\cdots+\widehat{A}_{s_{1}}^{h k}$ и $\widehat{B}_{1}^{h k}+\cdots+\widehat{B}_{s_{1}+s_{2}}^{h k}$, где $\widehat{A}_{l_{1}}^{h k}$, $\widehat{B}_{l_{1}}^{h k}$ и $\widehat{B}_{l_{2}}^{h k}$ вычисляются по формулам (1.12) и (1.14) через решения задач $(1.11)$ и (1.13), в которых 1 и 2 заменены на $l_{1}=1, \ldots, s_{1}$ и $l_{2}=s_{1}+1, \ldots, s_{1}+s_{2}$ соответственно. Когда выполнено условие (1.2), осредненная задача (1.21) содержит $s_{1}+s_{2}$ связанных систем уравнений относительно вектор-функций $v_{1}, \ldots, v_{s_{1}}+s_{2}$ и соответствующие начальные и граничные условия. В случае, когда выполнено условие (1.3), осредненная задача (1.22) содержит $s_{1}+s_{2}$ независимых задач относительно вектор-функций $v_{1}, \ldots, v_{s_{1}+s_{2}}$. Такие осредненные задачи принято называть многокомпонентными. Это определение введено Г. П. Панасенко [9], [10], в случае, когда множества $F_{1}, \ldots, F_{s_{1}+s_{2}}$ составлены из цилиндров. Этот случай является в некотором смысле прямым произведением рассматриваемых здесь случаев, поскольку каждый такой цилиндр является связньм в направлении образуюшей и вполне несвязен в перпендикулярных направлениях. Кроме того, в работах $[9],[10]$ предполагается, что $m_{0}$ имеет порядок $\sigma$, поэтому осредненные задачи из этих работ сушественно отличаются от осредненных задач, полученных здесь. Используемые в данной работе методы позволяют рассмотреть задачу (1.4) и в более общей ситуации, когда $m_{0}$ или $m_{0}$ и $r_{0}$ имеют порядок $\mu$, где $\mu$ является малым положительным параметром. Поскольку коэффициенты $m_{\varepsilon}$ и $r_{\varepsilon}$ соответствуют плотности, последний случай встречается в задачах, возникающих на практике, и будет рассмотрен здесь более подробно.

Пусть в условия (1.8) внесено изменение, состоящее в том, что

$$
m_{0}\left(\frac{x}{\varepsilon}\right)=\mu m\left(\frac{x}{\varepsilon}\right), \quad r_{0}\left(\frac{x}{\varepsilon}\right)=\mu r\left(\frac{x}{\varepsilon}\right) \text { в } \Omega_{0}^{\varepsilon}
$$

где $\mu \rightarrow 0$ и $m(y), r(y)$ являются 1 -периодическими ограниченными (почти всюду) функциями на $F_{0}$. Кроме того, функция $m(y)$ отделена от нуля и удовлетворяет неравенствам (1.9). Определим

$$
\widehat{M}=\left\langle m_{1}\right\rangle_{1}+\left\langle m_{2}\right\rangle_{\mathbf{2}}, \quad \widehat{R}=\left\langle r_{1}\right\rangle_{\mathbf{1}}+\left\langle r_{2}\right\rangle_{\mathbf{2}} .
$$


ТЕОРема 4. Пусть выполнены условия (1.1) и (1.23). Предположсим, что и является решением задачи (1.4) и $v$-решение задачи (1.15), в которой $M$ u $R$ заменены на $\widehat{M}, \widehat{R}$ соответственно. Тогда

$$
\max _{0 \leqslant t \leqslant T}\|u-v\|_{L^{2}\left(\Omega_{12}\right)^{n}}^{2} \leqslant C\left(\varepsilon+\sigma+\mu^{2}+\frac{\varepsilon^{2}}{\sigma}\right),
$$

әде постоянная $C$ не зависит от $\varepsilon, \sigma u \mu$.

В этой теореме разность $u-v$ оценивается только на множестве $\Omega_{12}^{\varepsilon} \equiv \Omega_{1}^{\varepsilon} \cup \Omega_{2}^{\varepsilon}$ в отличие от теоремы 1 , что связано со спецификой рассматриваемой задачи. Впрочем, и эта оценка дает представление об асимптотическом поведении решения задачи (1.4) в рассматриваемом случае. Кроме того, в процессе доказательства этой теоремы будет оценена “энергетическая" норма разности первообразных решения задачи (1.4) и начальных членов асимптотического разложения на всем множестве $\Omega$.

ТЕОРема 5. Пусть выполнены условия (1.2) и (1.23). Предположсим, что и является решением задачи (1.4) и $\left(v_{1}, v_{2}\right)$ - решение задачи $(1.21)$, в которой $M_{s}^{l}=0 u R_{s}^{0}=0$ для $s, l=1,2$. Тогда

$$
\max _{0 \leqslant t \leqslant T}\left\|u-E_{1 \varepsilon} v_{1}-E_{2 \varepsilon} v_{2}\right\|_{L^{2}\left(\Omega_{12}^{\varepsilon}\right)^{n}}^{2} \leqslant C\left(\varepsilon+\sigma+\mu^{2}+\left|\vartheta-\frac{\sigma}{\varepsilon^{2}}\right|\right),
$$

әде постоянная $C$ не зависит от $\varepsilon, \sigma u \mu$.

В случае, когда выполнены условия (1.3) и (1.23), предполагается также, что при $\varepsilon \rightarrow 0$ выполнены соотношения $\sigma \rightarrow 0, \mu \rightarrow 0$ и одно из следующих трех условий:

$$
\begin{aligned}
& \frac{\sigma}{\varepsilon^{2} \mu} \rightarrow \infty, \\
& \frac{\sigma}{\varepsilon^{2} \mu} \rightarrow \theta, \\
& \frac{\sigma}{\varepsilon^{2} \mu} \rightarrow 0,
\end{aligned}
$$

где $\theta$ - заданная положительная постоянная.

ТЕОРема 6. Пусть выполнены условия (1.3), (1.23) и (1.25). Предположсим, что и является решением задачи (1.4) и $v_{1}, v_{2}$-решение задач (1.22). Тогда

$$
\max _{0 \leqslant t \leqslant T}\left\|u-E_{1 \varepsilon} v_{1}-E_{2 \varepsilon} v_{2}\right\|_{L^{2}\left(\Omega_{12}^{\varepsilon}\right)^{n}}^{2} \leqslant C\left(\varepsilon+\sigma+\mu^{2}+\frac{\sigma}{\varepsilon^{2}}+\left(\frac{\varepsilon^{2} \mu}{\sigma}\right)^{2}\right),
$$

әде постоянная $C$ не зависит от $\varepsilon, \sigma u \mu$.

ТеОРема 7. Пусть выполнены условия (1.3), (1.23), (1.26), $f, g_{k} \in C_{0}^{\infty}((0, T)$ $\times \Omega)^{n}, w_{0}=0$ и $w_{1}=0$, где $k=1, \ldots, n$. Предположим, что и является решением задачи (1.4) и $v_{1}, v_{2}$ - решение задач (1.22). Тогда

$$
\max _{0 \leqslant t \leqslant T}\left\|u-E_{1 \varepsilon} v_{1}-E_{2 \varepsilon} v_{2}\right\|_{L^{2}\left(\Omega_{12}^{\varepsilon}\right)^{n}}^{2} \leqslant C\left(\varepsilon+\sigma+\mu^{2}+\frac{\sigma}{\varepsilon^{2}}+\left|\theta-\frac{\sigma}{\varepsilon^{2} \mu}\right|\right),
$$

әде постоянная $C$ не зависит от $\varepsilon, \sigma u \mu$. 
ТЕОРЕМа 8. Пусть выполнены условия (1.3), (1.23), (1.27) и $\nabla_{y}(\mathrm{r} / \mathrm{m}) \in$ $L^{\infty}\left(Y_{0}\right)^{n}$. Предположим, что и является решением задачи (1.4) $и v_{1}, v_{2}$ решение задач (1.22). Тогда

$$
\max _{0 \leqslant t \leqslant T}\left\|u-\chi_{1}^{\varepsilon} v_{1}-\chi_{2}^{\varepsilon} E_{2 \varepsilon} v_{2}\right\|_{L^{2}\left(\Omega_{12}^{\varepsilon}\right)^{n}}^{2} \leqslant C\left(\varepsilon+\sigma+\mu^{2}+\frac{\sigma}{\varepsilon^{2}}+\frac{\sqrt[4]{\sigma}}{\sqrt[4]{\mu} \sqrt{\varepsilon}}\right)
$$

где постоянная $C$ не зависит от $\varepsilon, \sigma u \mu$.

ЗАмЕчАниЕ 4. Вопросы осреднения стационарного варианта задачи (1.4) рассматривались в работах [4], [9] и [11]. Параболический вариант задачи (1.4) рассматривался в [12]. В работе [10] предложен алгоритм построения асимптотики решения задачи (1.4) в случае, когда $m_{0}$ имеет порядок $\sigma$. В работе [9] намечена схема обоснования этой асимптотики в предположении, что условия Дирихле из (1.4) заменены на условия периодичности и множества $F_{1}, \ldots$ являются цилиндрами.

Доказательства представленных здесь утверждений даны в $\S \S 4-10$. Несколько вспомогательных лемм, используемых в этих доказательствах, приведены в 22 . Частные случаи теорем 1-3 были анонсированы в заметке [13]. Утверждения, близкие к теореме 1, были ранее доказаны Н. С. Бахваловым.

Автор выражает признательность Н. С. Бахвалову за многочисленные полезные советы по рассматриваемьм в статье вопросам.

\section{§2. Леммы}

В дальнейшем изложении $C$ обозначает постоянные, не зависяшие от малых параметров $\varepsilon, \sigma$ и $\mu$. Кроме того, для заданной 1-периодической на $Y$ функции $P(y)$ через $P_{\varepsilon}=P(x / \varepsilon)$ обозначается $\varepsilon$-периодическое продолжение этой функции на $\Omega$. Более точно, $P(y)$ продолжается как 1 -периодическая функция на $\mathbb{R}^{n}$ и выбирается ограничение полученной функции при $y=x / \varepsilon$ на $\Omega$, которое и обозначается $P_{\varepsilon}$.

Следуя, например, [5; гл. 12], будем по возможности использовать безындексную форму записи рассматриваемых систем уравнений. Тогда задачу (1.4) можно переписать в виде

$$
\begin{gathered}
m_{\varepsilon} u_{t t}^{\prime \prime}-\operatorname{div}\left(A_{\sigma}^{\varepsilon} \nabla u\right)=r_{\varepsilon} f+\operatorname{div}\left(B_{\sigma}^{\varepsilon} g\right) \text { в } \Omega \times(0, T), \\
\left.u\right|_{t=0}=w_{0} \text { в } \Omega,\left.\quad u_{t}^{\prime}\right|_{t=0}=w_{1} \text { в } \Omega, \quad u=0 \text { на } \partial \Omega \times(0, T) .
\end{gathered}
$$

Из свойств симметрии (1.7) тензора $A_{\sigma}^{\varepsilon}$ следует, что

$$
A_{\sigma}^{\varepsilon} \nabla u=A_{\sigma}^{\varepsilon} e(u), \quad e(u)=\frac{1}{2}\left(\nabla u+\nabla u^{T}\right) .
$$

Действительно, например, на множестве $\Omega_{1}^{\varepsilon}$ для компонент матрицы $A_{\sigma}^{\varepsilon} \nabla u$ по определению имеем равенства

$$
\left(A_{\sigma}^{\varepsilon} \nabla u\right)_{j k}=a_{i j 1}^{h k} \frac{\partial u_{i}}{\partial x_{h}}=a_{h j 1}^{i k} \frac{\partial u_{i}}{\partial x_{h}}=a_{i j 1}^{h k} \frac{\partial u_{h}}{\partial x_{i}}=\left(A_{\sigma}^{\varepsilon} \nabla u^{T}\right)_{j k}
$$

которые доказывают (2.2). Здесь и далее $i, j, h, k=1, \ldots, n$. 
Из (1.7) также следует, что матрицы $A_{\sigma}^{\varepsilon} e(u)$ и $B_{\sigma}^{\varepsilon} g$ симметричны. Поэтому с учетом (2.2) интегральное тождество (1.10) можно переписать в более традиционном для теории упругости виде

$$
\int_{\Omega}\left(m_{\varepsilon} u_{t t}^{\prime \prime}, \varphi\right) d x+\int_{\Omega}\left(A_{\sigma}^{\varepsilon} e(u), e(\varphi)\right) d x=\int_{\Omega}\left(r_{\varepsilon} f, \varphi\right) d x-\int_{\Omega}\left(B_{\sigma}^{\varepsilon} g, e(\varphi)\right) d x
$$

где $\varphi \in H_{0}^{1}(\Omega)^{n}$ и $(\cdot, \cdot)$ обозначает скалярное произведение векторов или матриц. Например, для $A=\left\{a_{h k}\right\}$ и $B=\left\{b_{h k}\right\}$ из $\mathbb{R}^{n \times n}$ по определению $(A, B)=a_{h k} b_{h k}$ и $|A|^{2}=(A, A)$.

ЛЕмма 1. Для решений задачи (1.4) выполнено неравенство

$$
\begin{aligned}
\mathscr{E}(u) \equiv & \left\|u_{t}^{\prime}\right\|_{C^{0}\left([0, T] ; L^{2}(\Omega)^{n}\right)}^{2}+\sigma\|e(u)\|_{C^{0}\left([0, T] ; L^{2}\left(\Omega_{0}^{\varepsilon}\right)^{n \times n}\right)}^{2} \\
& +\|e(u)\|_{C^{0}\left([0, T] ; L^{2}\left(\Omega_{12}^{\varepsilon}\right)^{n \times n}\right)}^{2} \\
\leqslant & C\left(\|f\|_{L^{1}\left(0, T ; L^{2}(\Omega)^{n}\right)^{2}}^{2}+\sigma\left\|e\left(w_{0}\right)\right\|_{L^{2}\left(\Omega_{0}^{\varepsilon}\right)^{n \times n}}^{2}+\left\|e\left(w_{0}\right)\right\|_{L^{2}\left(\Omega_{12}^{\varepsilon}\right)^{n \times n}}^{2}+\left\|w_{1}\right\|_{L^{2}(\Omega)^{n}}^{2}\right. \\
& \left.+\|g\|_{C^{0}\left([0, T] ; L^{2}(\Omega)^{n \times n}\right)}^{2}+\left\|g_{t}^{\prime}\right\|_{L^{1}\left(0, T ; L^{2}(\Omega)^{n \times n}\right)}^{2}\right) .
\end{aligned}
$$

ДокАЗАТЕльство. Как уже отмечалось, для решений задачи (1.4) выполнены включения $u_{t}^{\prime} \in C^{0}\left([0, T] ; L^{2}(\Omega)^{n}\right)$ и $u \in C^{0}\left([0, T] ; H_{0}^{1}(\Omega)^{n}\right)$. Поэтому величина $\mathscr{E}(u)$ определена, и необходимо только выяснить зависимость соответствующих постоянных от $\varepsilon$ и $\sigma$. Выбирая $\varphi=u_{t}^{\prime}$ в тождестве (2.4), получаем

$$
\begin{aligned}
\left(\frac{1}{2} \int_{\Omega} m_{\varepsilon}\left|u_{t}^{\prime}\right|^{2} d x\right)_{t}^{\prime}+\left(\frac{1}{2} \int_{\Omega}\left(A_{\sigma}^{\varepsilon} e(u), e(u)\right) d x\right)_{t}^{\prime} & \\
& =\int_{\Omega}\left(r_{\varepsilon} f, u_{t}^{\prime}\right) d x-\int_{\Omega}\left(B_{\sigma}^{\varepsilon} g, e\left(u_{t}^{\prime}\right)\right) d x
\end{aligned}
$$

где учтены свойства симметрии тензора $A_{\sigma}^{\varepsilon}$. Интегрируя это равенство по $t$ и используя условия (1.7), (1.9), имеем

$$
\begin{aligned}
&\left\|u_{t}^{\prime}(t)\right\|_{L^{2}(\Omega)^{n}}^{2}+\sigma\|e(u(t))\|_{L^{2}\left(\Omega_{0}^{\varepsilon}\right)^{n \times n}}^{2}+\|e(u(t))\|_{L^{2}\left(\Omega_{12}^{\varepsilon}\right)^{n \times n}}^{2} \\
& \leqslant \frac{2}{\alpha}\left(\frac{\beta}{2}\left(\left\|w_{1}\right\|_{L^{2}(\Omega)^{n}}^{2}+\sigma\left\|e\left(w_{0}\right)\right\|_{L^{2}\left(\Omega_{0}^{\varepsilon}\right)^{n \times n}}^{2}+\left\|e\left(w_{0}\right)\right\|_{L^{2}\left(\Omega_{12}^{\varepsilon}\right)^{n \times n}}^{2}\right)\right. \\
&\left.+\left|\int_{0}^{t} \int_{\Omega}\left(r_{\varepsilon} f, u_{t}^{\prime}\right) d x d t\right|+\left|\int_{0}^{t} \int_{\Omega}\left(B_{\sigma}^{\varepsilon} g, e\left(u_{t}^{\prime}\right)\right) d x d t\right|\right) .
\end{aligned}
$$

Интегрируя по частям в последнем слагаемом этого неравенства, получаем

$$
\begin{aligned}
& \left|\int_{0}^{t} \int_{\Omega}\left(B_{\sigma}^{\varepsilon} g, e\left(u_{t}^{\prime}\right)\right) d x d t\right| \leqslant\left|\int_{\Omega}\left(B_{\sigma}^{\varepsilon} g(t), e(u(t))\right) d x\right| \\
& +\left|\int_{\Omega}\left(\left.B_{\sigma}^{\varepsilon} g\right|_{t=0}, e\left(w_{0}\right)\right) d x\right|+\left|\int_{0}^{t} \int_{\Omega}\left(B_{\sigma}^{\varepsilon} g_{t}^{\prime}, e(u)\right) d x d t\right|
\end{aligned}
$$


Воспользуемся для оценки интегралов в (2.5) и (2.6) неравенством Коши-Буняковского и " $\delta$-неравенством", записанным в виде $|a b| \leqslant(\delta / 2) a^{2}+b^{2} /(2 \delta)$ для положительного $\delta$ и произвольных $a, b$. Тогда

$$
\begin{aligned}
&\left|\int_{\Omega}\left(B_{\sigma}^{\varepsilon} g, e(u)\right) d x\right| \leqslant\left|\int_{\Omega_{0}^{\varepsilon}}\left(B_{\sigma}^{\varepsilon} g, e(u)\right) d x\right|+\left|\int_{\Omega_{12}^{\varepsilon}}\left(B_{\sigma}^{\varepsilon} g, e(u)\right) d x\right| \\
& \leqslant \sigma C\|g\|_{L^{2}\left(\Omega_{0}^{\varepsilon}\right)^{n \times n}}\|e(u)\|_{L^{2}\left(\Omega_{0}^{\varepsilon}\right)^{n \times n}}+C\|g\|_{L^{2}\left(\Omega_{12}^{\varepsilon}\right)^{n \times n}}\|e(u)\|_{L^{2}\left(\Omega_{12}^{\varepsilon}\right)^{n \times n}} \\
& \leqslant C\left(\frac{\sigma \delta}{2}\|e(u)\|_{C^{0}\left([0, T] ; L^{2}\left(\Omega_{0}^{\varepsilon}\right)^{n \times n}\right)}^{2}+\frac{\sigma}{2 \delta}\|g\|_{C^{0}\left([0, T] ; L^{2}\left(\Omega_{0}^{\varepsilon}\right)^{n \times n}\right)}^{2}\right. \\
&\left.+\frac{\delta}{2}\|e(u)\|_{C^{0}\left([0, T] ; L^{2}\left(\Omega_{12}^{\varepsilon}\right)^{n \times n}\right)}^{2}+\frac{1}{2 \delta}\|g\|_{C^{0}\left([0, T] ; L^{2}\left(\Omega_{12}^{\varepsilon}\right)^{n \times n}\right)}^{2}\right) \\
& \leqslant \frac{\alpha}{12} \mathscr{E}(u)+\frac{3 C^{2}}{\alpha}\|g\|_{C^{0}\left([0, T] ; L^{2}(\Omega)^{n \times n}\right)}^{2},
\end{aligned}
$$

где было выбрано $\delta=\alpha /(6 C)$ и учтено, что $\sigma<1$. Аналогично получаем

$$
\begin{aligned}
& \left|\int_{0}^{t} \int_{\Omega}\left(B_{\sigma}^{\varepsilon} g_{t}^{\prime}, e(u)\right) d x d t\right|+\left|\int_{0}^{t} \int_{\Omega}\left(r_{\varepsilon} f, u_{t}^{\prime}\right) d x d t\right| \\
& \leqslant \frac{\alpha}{6} \mathscr{E}(u)+C\left(\left\|g_{t}^{\prime}\right\|_{L^{1}\left(0, T ; L^{2}(\Omega)^{n \times n}\right)}^{2}+\|f\|_{L^{1}\left(0, T ; L^{2}(\Omega)^{n \times n}\right)}^{2}\right) .
\end{aligned}
$$

Используя (2.6)-(2.8) и неравенство

$$
\begin{aligned}
\left|\int_{\Omega}\left(\left.B_{\sigma}^{\varepsilon} g\right|_{t=0}, e\left(w_{0}\right)\right) d x\right| \leqslant C( & \|g\|_{C^{0}\left([0, T] ; L^{2}(\Omega)^{n \times n}\right)}^{2} \\
& \left.+\sigma\left\|e\left(w_{0}\right)\right\|_{L^{2}\left(\Omega_{0}^{\varepsilon}\right)^{n \times n}}^{2}+\left\|e\left(w_{0}\right)\right\|_{\left.L^{2}\left(\Omega_{12}^{\varepsilon}\right)^{n \times n}\right)}^{2}\right)
\end{aligned}
$$

перейдем к максимуму по $t \in[0, T]$ в неравенстве (2.5). Тогда из (2.5)-(2.8) следует неравенство леммы с дополнительньм слагаемым $(1 / 2) \mathscr{E}(u)$ в правой части. Это дополнительное слагаемое можно вычесть из левой части полученного неравенства, что завершает доказательство леммы 1.

Постоянные $C$ в неравенствах леммы 1 не зависят от $T$. Как уже отмечалось, $u \in C^{0}\left([0, T] ; L^{2}(\Omega)^{n}\right)$. Поэтому из представления $u(t)=w_{0}+\int_{0}^{t} u_{\tau}^{\prime}(\tau) d \tau$ следует оценка

$$
\begin{aligned}
\max _{0 \leqslant t \leqslant T}\|u\|_{L^{2}(\Omega)^{n}} & \leqslant\left\|w_{0}\right\|_{L^{2}(\Omega)^{n}}+\int_{0}^{T}\left\|u_{t}^{\prime}\right\|_{L^{2}(\Omega)^{n}} d t \\
& \leqslant\left\|w_{0}\right\|_{L^{2}(\Omega)^{n}}+T\left\|u_{t}^{\prime}\right\|_{C^{0}\left([0, T] ; L^{2}(\Omega)^{n}\right)} .
\end{aligned}
$$

Таким образом, в левую часть первого неравенства леммы 1 можно добавить в качестве слагаемого величину $\|u\|_{C^{0}\left([0, T] ; L^{2}(\Omega)^{n}\right)}^{2}$, но в этом случае постоянная $C$ из этого неравенства будет зависеть от $T$.

Будем обозначать через $H_{P}^{1}\left(Y_{1}\right)$ пространство Соболева 1-периодических функций из $H^{1}\left(Y_{1}\right)$ (см., например, [4], [5]). Эквивалентно $H_{P}^{1}\left(Y_{1}\right)$ определяется как пространство квадратично интегрируемых вместе с производньми первого порядка функций на многообразии $Y_{1}$ с границей $S_{1}$ класса $C^{2}$. Тогда [2] определены пространство $H_{P}^{1 / 2}\left(S_{1}\right)$ следов на $S_{1}$ элементов из $H_{P}^{1}\left(Y_{1}\right)$ и пространство 
$H_{P}^{-1 / 2}\left(S_{1}\right)$, сопряженное к $H_{P}^{1 / 2}\left(S_{1}\right)$. Аналогично определяются пространства $H_{P}^{1}\left(Y_{0}\right), H_{P}^{1}\left(Y_{2}\right), \ldots$ При этом $H_{P}^{1}\left(Y_{2}\right)=H^{1}\left(Y_{2}\right)$ и $H_{P}^{-1 / 2}\left(S_{2}\right)=H^{-1 / 2}\left(S_{2}\right)$ в силу предположений на множество $Y_{2}$ (см. рис. 1 и 2 ).

Кроме того, из результатов [3; гл. $3, \S 3.5]$ и $[14 ;$ гл. 1 , теорема 1.2$]$ следует формула интегрирования по частям

$$
-\int_{S_{0}}(W, \nu V) d s=\int_{Y_{0}}\left(W, \operatorname{div}_{y} V\right) d y+\int_{Y_{0}}\left(\nabla_{y} W, V\right) d y,
$$

где $W \in H_{P}^{1}\left(Y_{0}\right)^{n}, V \in L^{2}\left(Y_{0}\right)^{n \times n} \mathrm{c} \operatorname{div}_{y} V \in L^{2}\left(Y_{0}\right)^{n}$ и след $\nu V$ определен как элемент пространства $H_{P}^{-1 / 2}\left(S_{0}\right)^{n}$ :

$$
\|\nu V\|_{H_{P}^{-1 / 2}\left(S_{0}\right)^{n}}^{2} \leqslant C\left(\|V\|_{L^{2}\left(Y_{0}\right)^{n \times n}}^{2}+\left\|\operatorname{div}_{y} V\right\|_{L^{2}\left(Y_{0}\right)^{n}}^{2}\right) .
$$

Аналогичные неравенства для следа и формулы интегрирования по частям выполнены для множеств $Y_{1}$ и $Y_{2}$, но с обратным знаком у граничного интеграла, поскольку нормаль $\nu$ является внутренней для границы $S_{0}$ и внешней для границ $S_{1}$ и $S_{2}$. Отметим также, что для $V=V^{T}$ последний интеграл в (2.10) можно переписать в виде

$$
\int_{Y_{0}}\left(\nabla_{y} W, V\right) d y=\int_{Y_{0}}\left(e_{y}(W), V\right) d y, \quad e_{y}(W)=\frac{1}{2}\left(\nabla_{y} W+\nabla_{y} W^{T}\right) .
$$

Известно [4], [5], что решения $N_{1 a}^{p}, N_{1 b}^{p} \in\left(H_{P}^{1}\left(Y_{1}\right) \cap L^{2}\left(Y_{1}\right) / \mathbb{R}\right)^{n \times n}$ задач (1.11) и (1.13) сушествуют и определењы однозначно, где $p=1, \ldots, n$. Эти утверждения вьполнены и при более слабых предположениях на $S_{1}$, но в дальнейшем встретятся более общие задачи Неймана на $Y_{1}$. Гладкость $S_{1}$ сушественна в доказательстве следуюшего известного (см., например, [2], [3]) утверждения.

Лемма 2. Пусть $V \in L^{2}\left(Y_{1}\right)^{n}, W \in H_{P}^{-1 / 2}\left(S_{1}\right)^{n}$ u $L \in L^{2}\left(Y_{1}\right)^{n \times n}$ удовлетворяют равенству $\int_{Y_{1}} V d y=\int_{S_{1}} W d s$. Тогда существует единственное решение $U \in\left(H_{P}^{1}\left(Y_{1}\right) \cap L^{2}\left(Y_{1}\right) / \mathbb{R}\right)^{n}{ }^{n} 1$-периодической задачи Неймана

$$
-\operatorname{div}_{y}\left(A_{1} \nabla_{y} U\right)=V+\operatorname{div}_{y} L \quad \text { в } Y_{1}, \quad-\nu\left(A_{1} \nabla_{y} U\right)=W+\nu L \text { на } S_{1},
$$

удовлетворяющее неравенству

$\|U\|_{L^{2}\left(Y_{1}\right)^{n}}+\left\|\nabla_{y} U\right\|_{L^{2}\left(Y_{1}\right)^{n \times n}} \leqslant C\left(\|V\|_{L^{2}\left(Y_{1}\right)^{n}}+\|W\|_{H_{P}^{-1 / 2}\left(S_{1}\right)^{n}}+\|L\|_{L^{2}\left(Y_{1}\right)^{n \times n}}\right)$.

В дальнейшем используется также аналогичное утверждение для задачи Неймана на $Y_{2}$. Это утверждение формулируется несколько сложнее, поскольку в силу условий на $Y_{2}$ ядро и коядро такой задачи совпадают с $\widetilde{n}$-мерньм линейным пространством $\Re$ жестких перемешений в $\mathbb{R}^{n}$. Напомним, что базис в $\Re$ образуют векторы $e_{1}, \ldots, e_{\widetilde{n}}$, составляющие матрицу $E_{2}$ и удовлетворяюшие условиям нормировки (1.17). Гладкость $S_{2}$ сушественна в доказательстве следуюшего известного (см., например, [2], [3]) утверждения. 
Лемма 3. Пусть $V \in L^{2}\left(Y_{2}\right)^{n}, W \in H^{-1 / 2}\left(S_{2}\right)^{n}$ u $L \in L^{2}\left(Y_{2}\right)^{n \times n}$ удовлетворяют равенствам

$$
-\int_{Y_{2}}\left(\nabla_{y} e_{l}, L\right) d y+\int_{Y_{2}}\left(e_{l}, V\right) d y=\int_{S_{2}}\left(e_{l}, W\right) d s \quad \partial \Omega_{s} \quad l=1, \ldots, \widetilde{n} .
$$

Тогда существует единственное решение $U \in H^{1}\left(Y_{2}\right)^{n} \cap L^{2}\left(Y_{2}\right)^{n} / \Re$ задачи

$$
-\operatorname{div}_{y}\left(A_{2} \nabla_{y} U\right)=V+\operatorname{div}_{y} L \quad \text { в } Y_{2}, \quad-\nu\left(A_{2} \nabla_{y} U\right)=W+\nu L \text { на } S_{2},
$$

удовлетворяющее неравенству

$$
\|U\|_{L^{2}\left(Y_{2}\right)^{n}}+\left\|\nabla_{y} U\right\|_{L^{2}\left(Y_{2}\right)^{n \times n}} \leqslant C\left(\|V\|_{L^{2}\left(Y_{2}\right)^{n}}+\|W\|_{H_{P}^{-1 / 2}\left(S_{2}\right)^{n}}+\|L\|_{L^{2}\left(Y_{2}\right)^{n \times n}}\right) .
$$

Отметим, что для $L=L^{T}$ первый интеграл в (2.13) равен нулю:

$$
\int_{Y_{2}}\left(\nabla_{y} \Psi, L\right) d y=\int_{Y_{2}}\left(e_{y}(\Psi), L\right) d y=0
$$

поскольку $e_{y}(\Psi)=0$ для $\Psi \in \Re$. Поэтому из свойств симметрии (1.7) для тензора $B_{\sigma}^{\varepsilon}$ и леммы 3 следует, что решения $N_{2 b}^{p} \in\left(H^{1}\left(Y_{2}\right)^{n} \cap L^{2}\left(Y_{2}\right)^{n} / \Re\right)^{n}$ второй задачи из (1.13) существуют и определены однозначно, где $p=1, \ldots, n$.

Известно [2], [3], что решения $P, Q_{1} \in C^{0}\left([0, T] ; H_{P}^{1}(Y)^{n \times n}\right)$ и $Q_{2} \in C^{0}([0, T]$; $\left.H_{P}^{1}(Y)^{n \times \widetilde{n}}\right)$ задач (1.19) существуют и определены однозначно. С учетом неравенства Корна (см., например, [3]) доказательство следующего утверждения повторяет доказательство леммы 1.

Лемма 4. Для решений задач (1.19) выполнены неравенства

$$
\begin{gathered}
\left\|P_{t}^{\prime}\right\|_{C^{0}\left([0, T] ; L^{2}(Y)^{n \times n}\right)}^{2}+\vartheta\left\|\nabla_{y} P\right\|_{C^{0}\left([0, T] ; L^{2}(Y)^{n \times n \times n}\right)}^{2} \leqslant C, \\
\left\|Q_{1 t}^{\prime}\right\|_{C^{0}\left([0, T] ; L^{2}(Y)^{n \times n}\right)}^{2}+\vartheta\left\|\nabla_{y} Q_{1}\right\|_{C^{0}\left([0, T] ; L^{2}(Y)^{n \times n \times n}\right)}^{2} \leqslant C, \\
\left\|Q_{2 t}^{\prime}\right\|_{C^{0}\left([0, T] ; L^{2}(Y)^{n \times \widetilde{n}}\right)}^{2}+\vartheta\left\|\nabla_{y} Q_{2}\right\|_{C^{0}\left([0, T] ; L^{2}(Y)^{n \times \widetilde{n} \times n)}\right.}^{2} \leqslant C,
\end{gathered}
$$

әде постоянная $C$ не зависит от $\vartheta$ и $T$.

Асимптотические свойства решений задач (1.19) при больших и малых $\vartheta$ также используются в дальнейшем. Для точной формулировки соответствуюших утверждений понадобятся некоторые дополнительные обозначения.

Определим $p, q_{1} \in H_{P}^{1}(Y)^{n \times n}$ и $q_{2} \in H_{P}^{1}(Y)^{n \times \widetilde{n}}$ как 1-периодические решения задач Дирихле

$$
\begin{gathered}
-\operatorname{div}_{y}\left(A_{0} \nabla_{y} p\right)=r_{0} E \text { в } Y_{0}, \quad p=0 \text { на } \bar{Y}_{12}, \\
-\operatorname{div}_{y}\left(A_{0} \nabla_{y} q_{s}\right)=m_{0} E_{s} \text { в } Y_{0}, \quad q_{s}=0 \text { на } \bar{Y}_{12}, \quad s=1,2 .
\end{gathered}
$$

Далее, введем обозначения для первообразных по $t$ от решений задач (1.19):

$$
\dot{P}=\int_{0}^{t} P d \tau, \quad \dot{Q}_{1}=\int_{0}^{t} Q_{1} d \tau, \quad \dot{Q}_{2}=\int_{0}^{t} Q_{2} d \tau .
$$


Лемма 5. Пусть $\omega=1 / \vartheta$ является малым параметром в задачах (1.19). Тогда

$$
\begin{gathered}
\|\dot{P}-\omega p\|_{L^{2}\left((0, T) ; L^{2}\left(Y_{0}\right)^{n \times n}\right)}+\left\|\nabla_{y}(\dot{P}-\omega p)\right\|_{L^{2}\left((0, T) ; L^{2}\left(Y_{0}\right)^{n \times n \times n}\right)} \leqslant C \omega^{2}(1+T), \\
\left\|\dot{Q}_{1}-\omega q_{1}\right\|_{L^{2}\left((0, T) ; L^{2}\left(Y_{0}\right)^{n \times n}\right)}+\left\|\nabla_{y}\left(\dot{Q}_{1}-\omega q_{1}\right)\right\|_{L^{2}\left((0, T) ; L^{2}\left(Y_{0}\right)^{n \times n \times n}\right)} \leqslant C \omega^{2}(1+T), \\
\left\|\dot{Q}_{2}-\omega q_{2}\right\|_{L^{2}\left((0, T) ; L^{2}\left(Y_{0}\right)^{n \times \widetilde{n}}\right)}+\left\|\nabla_{y}\left(\dot{Q}_{2}-\omega q_{2}\right)\right\|_{L^{2}\left((0, T) ; L^{2}\left(Y_{0}\right)^{n \times \tilde{n} \times n)}\right.} \leqslant C \omega^{2}(1+T),
\end{gathered}
$$

где постоянная $C$ не зависит от $\omega$ и $T$.

ДоказАтеЛЬСтво. Рассмотрим, например, первую задачу из (1.19). Интегрируя по $t$ уравнения этой задачи и учитывая (2.15), для $R=\dot{P}-\omega p$ получаем уравнения

$$
\operatorname{div}_{y}\left(A_{0} \nabla_{y} R\right)=\omega m_{0} P_{t}^{\prime} \text { в } Y_{0} \times(0, \infty), \quad R=0 \text { на } \bar{Y}_{12} \times(0, \infty) .
$$

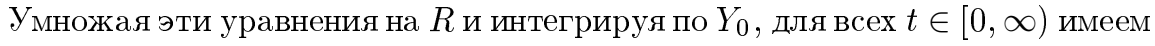

$$
\left\|\nabla_{y} R\right\|_{L^{2}\left(Y_{0}\right)^{n \times n \times n}}^{2} \leqslant \omega C\left\|P_{t}^{\prime}\right\|_{L^{2}(Y)^{n \times n}}\|R\|_{L^{2}(Y)^{n \times n}} \leqslant \omega C\left\|\nabla_{y} R\right\|_{L^{2}(Y)^{n \times n \times n}},
$$

где учтены лемма 4 и неравенство Фридрихса (см., например, [2]). Поэтому

$$
\|R\|_{C^{0}\left([0, T] ; L^{2}\left(Y_{0}\right)^{n \times n}\right)} \leqslant C \omega,
$$

где постоянная $C$ не зависит от $\omega$ и $T$. Аналогично, умножая уравнения из (2.16) на $R$ и интегрируя по $Y_{0} \times(0, T)$, имеем

$$
\begin{aligned}
& \int_{0}^{T} \int_{Y_{0}}\left(A_{0} \nabla_{y} R, \nabla_{y} R\right) d y d t \\
& \quad=\omega \int_{Y_{0}}\left(m_{0} P(T), R(T)\right) d y-\omega \int_{0}^{T} \int_{Y_{0}}\left(m_{0} P, R_{t}^{\prime}\right) d y d t \\
& \quad \leqslant \omega C\|P\|_{C^{0}\left([0, T] ; L^{2}\left(Y_{0}\right)^{n \times n}\right)}\|R\|_{C^{0}\left([0, T] ; L^{2}\left(Y_{0}\right)^{n \times n}\right)}+\omega C \int_{0}^{T}\|P\|_{L^{2}\left(Y_{0}\right)^{n \times n}}^{2} d t \\
& \quad \leqslant \omega^{2} C+\omega C \int_{0}^{T}\left\|\nabla_{y} P\right\|_{L^{2}\left(Y_{0}\right)^{n \times n \times n}}^{2} d t
\end{aligned}
$$

поскольку $R_{t}^{\prime}=P$. Теперь лемма 5 следует из леммы 4 .

Лемма 6. Пусть $\nabla_{y}\left(r_{0} / m_{0}\right) \in L^{\infty}(Y)$ u $\omega=\sqrt{\vartheta}$ является малым параметром в задачах (1.19). Тогда

$$
\begin{aligned}
\left\|P_{t}^{\prime}-\frac{r_{0}}{m_{0}} E\right\|_{C^{0}\left([0, T] ; L^{2}\left(Y_{0}\right)^{n \times n}\right)} & +\left\|Q_{1 t}^{\prime}-E_{1}\right\|_{C^{0}\left([0, T] ; L^{2}\left(Y_{0}\right)^{n \times n}\right)} \\
+\| Q_{2 t}^{\prime}- & -E_{2} \|_{C^{0}\left([0, T] ; L^{2}\left(Y_{0}\right)^{n \times \widetilde{n}}\right)} \leqslant C \sqrt{\omega}(1+T),
\end{aligned}
$$

әде постоянная $C$ не зависит от $\omega$ и $T$. 
ДокаЗАТЕльство. Рассмотрим, например, первую задачу из (1.19). Определим гладкую в $Y_{0}$ функцию $\eta_{\omega}$, равную нулю при $\operatorname{dist}\left(y, S_{0}\right) \leqslant \omega$ и единице при $\operatorname{dist}\left(y, S_{0}\right) \geqslant 2 \omega$, где $\operatorname{dist}\left(y, S_{0}\right)$ обозначает расстояние от точки $y \in Y_{0}$ до границы $S_{0}=\partial Y_{0}$. Для достаточно малых $\omega\left(\leqslant \omega_{0}\right.$ для некоторого положительного $\left.\omega_{0}\right)$ такие функции существуют, $\omega\left|\nabla_{y} \eta_{\omega}\right| \leqslant C$ в $Y_{0}$, и носители функций $\eta_{\omega}-1$ и $\nabla_{y} \eta_{\omega}$ содержатся в приграничной полосе $Y_{2 \omega} \subset Y_{0}$ ширины $2 \omega$ (см., например, [4]). Продолжим функцию $\eta_{\omega}$ нулем на $Y_{1}$ и $Y_{2}$. Тогда из ограниченности множества $Y$ и известной оценки $\operatorname{mes}\left(Y_{2 \omega}\right) \leqslant C \omega$ следуют неравенства

$$
\left\|\eta_{\omega}-1\right\|_{L^{2}\left(Y_{0}\right)}^{2} \leqslant C \omega, \quad\left\|\nabla_{y} \eta_{\omega}\right\|_{L^{2}\left(Y_{0}\right)^{n}}^{2} \leqslant \frac{C}{\omega}
$$

Пусть матричнозначная функция $P_{\omega}(t, y)$ является 1-периодическим решением начально-краевой задачи на $Y_{0}$ :

$$
\begin{gathered}
m_{0}\left(P_{\omega}\right)_{t t}^{\prime \prime}-\omega^{2} \operatorname{div}_{y}\left(A_{0} \nabla_{y} P_{\omega}\right)=0 \text { в } Y_{0} \times(0, \infty), \\
\left.P_{\omega}\right|_{t=0}=0 \text { в } Y_{0},\left.\quad\left(P_{\omega}\right)_{t}^{\prime}\right|_{t=0}=E_{\omega} \text { в } Y_{0}, \quad P_{\omega}=0 \text { на } \bar{Y}_{12} \times(0, \infty),
\end{gathered}
$$

где $E_{\omega}=\eta_{\omega}\left(r_{0} / m_{0}\right) E$. Тогда, используя $(2.17)$ и повторяя доказательство леммы 1 , получаем

$$
\left\|\left(P-P_{\omega}\right)_{t}^{\prime}\right\|_{C^{0}\left([0, T] ; L^{2}(Y)^{n \times n}\right)}^{2} \leqslant C\left\|\eta_{\omega}-1\right\|_{L^{2}\left(Y_{0}\right)}^{2} \leqslant C \omega .
$$

Определим $U_{\omega}=P_{\omega}-t E_{\omega}$. Тогда для $U_{\omega}$ имеем начально-краевую задачу

$$
\begin{aligned}
& m_{0}\left(U_{\omega}\right)_{t t}^{\prime \prime}-\omega^{2} \operatorname{div}_{y}\left(A_{0} \nabla_{y} U_{\omega}\right)=\omega^{2} t \operatorname{div}_{y}\left(A_{0} \nabla_{y} E_{\omega}\right) \text { в } Y_{0} \times(0, \infty), \\
& \left.U_{\omega}\right|_{t=0}=0 \text { в } Y_{0},\left.\quad\left(U_{\omega}\right)_{t}^{\prime}\right|_{t=0}=0 \text { в } Y_{0}, \quad U_{\omega}=0 \text { на } \bar{Y}_{12} \times(0, \infty) .
\end{aligned}
$$

Следовательно, используя (2.17) и условия леммы, получаем

$$
\begin{aligned}
& \left\|\left(P_{\omega}\right)_{t}^{\prime}-E_{\omega}\right\|_{C^{0}\left([0, T] ; L^{2}(Y)^{n \times n}\right)}^{2}=\left\|\left(U_{\omega}\right)_{t}^{\prime}\right\|_{C^{0}\left([0, T] ; L^{2}(Y)^{n \times n}\right)}^{2} \\
& \quad \leqslant \omega^{2} C\left\|t \nabla_{y} E_{\omega}\right\|_{C^{0}\left([0, T] ; L^{2}(Y)^{n \times n \times n}\right)}^{2}+\omega^{2} C\left\|\nabla_{y} E_{\omega}\right\|_{L^{1}\left(0, T ; L^{2}(Y)^{n \times n \times n}\right)}^{2} \\
& \quad \leqslant \omega C\left(T^{2}+T\right)
\end{aligned}
$$

(см. доказательство леммы 1 для $\left.g=t \nabla_{y} E_{\omega}\right)$.

Таким образом, учитывая $(2.17),(2.18)$ и неравенство треугольника, имеем

$$
\begin{aligned}
\| P_{t}^{\prime}- & \left(r_{0} / m_{0}\right) E \|_{C^{0}\left([0, T] ; L^{2}(Y)^{n \times n}\right)} \\
\leqslant & \left\|\left(P-P_{\omega}\right)_{t}^{\prime}\right\|_{C^{0}\left([0, T] ; L^{2}(Y)^{n \times n}\right)} \\
& \quad+\left\|\left(P_{\omega}\right)_{t}^{\prime}-E_{\omega}\right\|_{C^{0}\left([0, T] ; L^{2}(Y)^{n \times n}\right)}+\left\|E_{\omega}-\left(r_{0} / m_{0}\right) E\right\|_{C^{0}\left([0, T] ; L^{2}\left(Y_{0}\right)^{n \times n}\right)} \\
\leqslant & C \sqrt{\omega}\left(1+\sqrt{T^{2}+T}\right) \leqslant C \sqrt{\omega}(1+T),
\end{aligned}
$$

что доказывает лемму 6.

Напомним, что свертка по $t$ от гладких функций $P(t, x)$ и $v(t, x)$ определяется равенствами

$$
(P * v)(t)=\int_{0}^{t} P(t-\tau) v(\tau) d \tau=\int_{0}^{t} P(\tau) v(t-\tau) d \tau
$$

и выполняются формулы дифференцирования

$$
(P * v)_{t}^{\prime}=\left(\left.P\right|_{t=0}\right) v+\left(P_{t}^{\prime}\right) * v=P\left(\left.v\right|_{t=0}\right)+P *\left(v_{t}^{\prime}\right) .
$$

Это определение можно продолжить достаточно стандартным образом на более общие классы функций и распределений (см., например, [15]). 
Лемма 7. Пусть $P \in L^{2}\left(0, T ; L^{2}(\Omega)\right) \quad u \quad v \in L^{2}\left(0, T ; L^{\infty}(\Omega)\right) . \quad$ Тогда $P * v \in L^{\infty}\left(0, T ; L^{2}(\Omega)\right)$. Kроме того, $P * v \in C^{0}\left([0, T] ; L^{2}(\Omega)\right)$, если $v_{t}^{\prime} \in L^{2}\left(0, T ; L^{\infty}(\Omega)\right)$.

ДоказАТЕльство. Из неравенства Коши-Буняковского получаем

$$
\begin{aligned}
\|(P * v)(t)\|_{L^{2}(\Omega)} & \leqslant \int_{0}^{t}\|P(t-\tau) v(\tau)\|_{L^{2}(\Omega)} d \tau \\
& \leqslant \int_{0}^{t}\|P(t-\tau)\|_{L^{2}(\Omega)}\|v(\tau)\|_{L^{\infty}(\Omega)} d \tau \\
& \leqslant\|P\|_{L^{2}\left(0, T ; L^{2}(\Omega)\right)}\|v\|_{L^{2}\left(0, T ; L^{\infty}(\Omega)\right)}
\end{aligned}
$$

что доказывает первую часть леммы 7. В соответствии со вторым равенством из $(2.20)$ имеем $(P * v)_{t}^{\prime} \in L^{2}\left(0, T ; L^{2}(\Omega)\right)$, если $v_{t}^{\prime} \in L^{2}\left(0, T ; L^{\infty}(\Omega)\right)$. Тогда лемма 7 следует из теоремы "вложения" (см., например, [14; гл. 3, лемма 1.2]).

Лемма 8. Пусть $P \in L^{1}(0, T) \quad u \quad v \in L^{2}\left(0, T ; L^{2}(\Omega)\right) . \quad$ Тогда $P * v \in$ $L^{2}\left(0, T ; L^{2}(\Omega)\right)$.

ДокаЗАТЕЛьСтво. Выберем $h \in L^{2}\left(0, T ; L^{2}(\Omega)\right)$ с $\|h\|_{L^{2}\left(0, T ; L^{2}(\Omega)\right)}=1$ и продолжим $P, v, h$ нулем при $t \in \mathbb{R} \backslash[0, T]$. Тогда, используя теорему Фубини и инвариантность нормы в пространстве $L^{2}\left(\mathbb{R} ; L^{2}(\Omega)\right)$ относительно сдвигов по $t$, получаем

$$
\begin{aligned}
\left|\int_{-\infty}^{\infty} \int_{\Omega}(P * v)(t) h(t) d x d t\right| & \leqslant \int_{-\infty}^{\infty} \int_{\Omega} \int_{-\infty}^{t}|P(\tau)||v(t-\tau)||h(t)| d \tau d x d t \\
& \leqslant \int_{-\infty}^{\infty}|P(\tau)| \int_{\Omega} \int_{-\infty}^{\infty}|v(t-\tau)||h(t)| d t d x d \tau \\
& \leqslant \int_{0}^{T}|P(\tau)| d \tau\|v\|_{L^{2}\left(0, T ; L^{2}(\Omega)\right)} .
\end{aligned}
$$

Супремум по $h$ от левой части этого неравенства задает норму в пространстве $L^{2}\left(0, T ; L^{2}(\Omega)\right)$, что доказывает лемму 8 .

Фиксируем $\gamma>0$ и определим пространство $L_{\gamma}^{2}\left(0, \infty ; L^{2}(\Omega)\right)$ как множество функций из $L_{\mathrm{loc}}^{2}\left(0, \infty ; L^{2}(\Omega)\right)$, для которых конечна величина

$$
\|u\|_{L_{\gamma}^{2}\left(0, \infty ; L^{2}(\Omega)\right)}=\left\|e^{-\gamma t} u\right\|_{L^{2}\left(0, \infty ; L^{2}(\Omega)\right)} .
$$

Последнее равенство определяет норму в пространстве $L_{\gamma}^{2}\left(0, \infty ; L^{2}(\Omega)\right)$, относительно которой это пространство является полным (см., например, [16]).

Следуя [16], определим пространство $E_{\gamma}\left(L^{2}(\Omega)\right)$ как множество функций $U(\rho)=$ $U\left(\rho_{1}+i \rho_{2}\right)$ со значениями в $L^{2}(\Omega)$, заданњых и голоморфных в комплексной полуплоскости $\mathbb{C}_{\gamma}=\left\{\rho \in \mathbb{C}: \rho=\rho_{1}+i \rho_{2}, \rho_{1}>\gamma\right\}$, для которых конечна величина

$$
\|U(\rho)\|_{E_{\gamma}\left(L^{2}(\Omega)\right)}^{2}=\int_{-\infty}^{\infty}\left\|U\left(\gamma+i \rho_{2}\right)\right\|_{L^{2}(\Omega)}^{2} d \rho_{2},
$$

где интеграл определяется как предел в среднем интеграла от $K$ до $-K$ при $K \rightarrow \infty$. Последнее равенство определяет норму в пространстве $E_{\gamma}\left(L^{2}(\Omega)\right)$. Следуюший вариант теоремы Пэли-Винера доказан в [16]. 
ТЕОРемА 9. Преобразование Лапласа

$$
\widehat{u(t)}=\int_{0}^{\infty} e^{-\rho t} u(t) d t=U(\rho)
$$

отображсает пространство $L_{\gamma}^{2}\left(0, \infty ; L^{2}(\Omega)\right)$ на пространство $E_{\gamma}\left(L^{2}(\Omega)\right)$ взаимно однозначно и взаимно непрерывно.

Аналогично определяются пространства $E_{\gamma}\left(H_{0}^{1}(\Omega)\right)$ и $E_{\gamma}\left(L^{2}(Y)\right)$, для которых также выполнен аналог теоремы 9. Кроме того [16], преобразование Лапласа коммутирует с дифференцированиями по пространственным переменным и оператор свертки по $t$ переводится преобразованием Лапласа в оператор поточечного умножения.

ЛЕмма 10. Пусть выполнены условия теоремы 2. Тогда существует единственное достаточно гладкое решение начально-краевой задачи (1.21), причем константы в оценках соответствующих норм не зависят от $\vartheta$.

ДокАЗАтЕльство. Рассмотрим сначала более простой случай $Y_{2}=\varnothing$. Тогда в соответствии с замечанием 3 задача (1.21) принимает вид

$$
\begin{gathered}
M_{1} v_{t t}^{\prime \prime}-M_{1}^{1} *\left(v_{t t}^{\prime \prime}\right)-\operatorname{div}\left(\widehat{A}_{1} \nabla v\right)=R_{1} f-R_{1}^{0} * f+\operatorname{div}\left(\widehat{B}_{1} g\right) \text { в } \Omega \times(0, \infty), \\
\left.v\right|_{t=0}=0 \text { в } \Omega,\left.\quad v_{t}^{\prime}\right|_{t=0}=0 \text { в } \Omega, \quad v=0 \text { на } \partial \Omega \times(0, \infty),
\end{gathered}
$$

где $v=v_{1}, v_{2}=0$, использованы обозначения $(1.12),(1.14),(1.20)$ и финитные по $t$ функции $f, g$ продолжены нулем при $t \notin(0, T)$.

Для доказательства сушествования и единственности решения в подходящих пространствах, как обычно, наиболее сушественный момент - это априорные оценки (см., например, [2], [3]). В соответствии с теоремой 9 эти оценки можно попытаться доказать для преобразования Лапласа от решения задачи (2.21). Введем обозначения $V=\widehat{v}, F=\widehat{f}, G=\widehat{g}$ для преобразований Лапласа от функций $v, f$ и $g$ из (2.21). Тогда, применяя преобразование Лапласа к $(2.21)$, получаем краевую задачу для $V$ с комплексным параметром $\rho$ :

$$
\rho^{2} M V-\rho^{2} m V-\operatorname{div}(A \nabla V)=R F-r F+\operatorname{div}(B G) \text { в } \Omega, \quad V=0 \text { на } \partial \Omega
$$

(см., например, [16], [17]), где использованы обозначения

$$
\begin{gathered}
m=\widehat{M}_{1}^{1}=\rho^{2}\left\langle m_{0} E^{T} q\right\rangle_{\mathbf{o}}-\left\langle\left. m_{0} E^{T}\left(Q_{1 t}^{\prime}\right)\right|_{t=0}\right\rangle_{\mathbf{o}}=\rho^{2}\left\langle m_{0} E^{T} q\right\rangle_{\mathbf{o}}-\left\langle m_{0} E^{T} E\right\rangle_{\mathbf{o}} \\
r=\widehat{R}_{1}^{0}=\rho^{2}\left\langle m_{0} E^{T} p\right\rangle_{\mathbf{o}}-\left\langle r_{0} E^{T} E\right\rangle_{\mathbf{o}} \\
p=\widehat{P}, \quad q=\widehat{Q}_{1}, \quad E=E_{1}, \quad M=M_{1}, \quad A=\widehat{A}_{1}, \quad B=\widehat{B}_{1} .
\end{gathered}
$$

Отметим, что в силу леммы 4 функции $q(\rho), p(\rho)$ и поэтому $m(\rho), r(\rho)$ определены и являются голоморфными функциями на $\mathbb{C}_{0}$ со значениями в соответствующих пространствах (см., например, [17; гл. 6]). Из (1.19) получаем для $p(\rho)$ краевую задачу

$$
m_{0} \rho^{2} p-\vartheta \operatorname{div}_{y}\left(A_{0} \nabla_{y} p\right)=r_{0} E \text { в } Y_{0}, \quad p=0 \text { на } \bar{Y}_{12}
$$




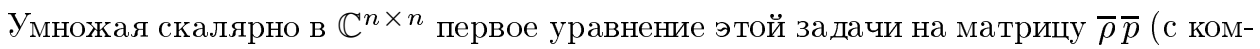
понентами, комплексно сопряженными к компонентам матрищы $\rho p)$ и интегрируя полученное соотношение по $Y_{0}$, имеем

$$
\rho\left\langle\left(m_{0} \rho p, \bar{\rho} \bar{p}\right)\right\rangle_{\mathbf{o}}+\vartheta \bar{\rho}\left\langle\left(A_{0} \nabla_{y} p, \nabla_{y} \bar{p}\right)\right\rangle_{\mathbf{o}}=\left\langle\left(r_{0} E, \bar{\rho} \bar{p}\right)\right\rangle_{\mathbf{o}} .
$$

Далее, рассмотрим уравнение, комплексно сопряженное к первому уравнению из (2.24). Умножая это уравнение на $\rho p$ и интегрируя по $Y_{0}$, получаем

$$
\bar{\rho}\left\langle\left(m_{0} \bar{\rho} \bar{p}, \rho p\right)\right\rangle_{\mathbf{o}}+\vartheta \rho\left\langle\left(A_{0} \nabla_{y} \bar{p}, \nabla_{y} p\right)\right\rangle_{\mathbf{o}}=\left\langle\left(r_{0} E, \rho p\right)\right\rangle_{\mathbf{o}}
$$

Учитывая свойства симметрии (1.7) тензора $A_{0}$, сложим соотношения $(2.25)$ и (2.26). Тогда $\rho_{1}\left\langle\left(m_{0} \rho p, \bar{\rho} \bar{p}\right)\right\rangle_{\mathbf{o}}+\vartheta \rho_{1}\left\langle\left(A_{0} \nabla_{y} p, \nabla_{y} \bar{p}\right)\right\rangle_{\mathbf{o}}=\operatorname{Re}\left\langle\left(r_{0} E, \bar{\rho} \bar{p}\right)\right\rangle_{\mathbf{o}}$, где по определению $\operatorname{Re} a=(a+\bar{a}) / 2$, и поэтому $|\operatorname{Re} a| \leqslant(|a|+|\bar{a}|) / 2=|a|$ для $a \in \mathbb{C}$. Следовательно, для всех $\rho=\rho_{1}+i \rho_{2}$ с $\rho_{1} \geqslant 1$ имеем неравенство

$$
\|\rho p\|_{L^{2}\left(Y_{0}\right)^{n \times n}}^{2} \leqslant \frac{1}{\alpha}\left|\operatorname{Re}\left\langle\left(r_{0} E, \bar{\rho} \bar{p}\right)\right\rangle_{\mathbf{o}}\right| \leqslant \frac{1}{\alpha}\left\|r_{0} E\right\|_{L^{2}\left(Y_{0}\right)^{n \times n}}\|\rho p\|_{L^{2}\left(Y_{0}\right)^{n \times n}} .
$$

Таким образом, используя обозначения (2.23), заключаем, что

$$
|r| \leqslant\left|\left\langle r_{0} E^{T} E\right\rangle_{\mathbf{o}}\right|+|\rho|\left|\left\langle m_{0} E^{T} \rho p\right\rangle_{\mathbf{o}}\right| \leqslant C(1+|\rho|),
$$

где постоянная $C$ не зависит от $\vartheta$ и $\rho \in \mathbb{C}_{1}$.

В соответствии с (1.19) краевая задача для $q(\rho)$ имеет вид

$$
m_{0} \rho^{2} q-\vartheta \operatorname{div}_{y}\left(A_{0} \nabla_{y} q\right)=m_{0} E \text { в } Y_{0}, \quad q=0 \text { на } \bar{Y}_{12} .
$$

Умножая матрично в $\mathbb{C}^{n \times n}$ первое уравнение этой задачи на $\bar{\rho}^{2} \bar{q}^{T}$ и интегрируя по $Y_{0}$, имеем $\left\langle m_{0} \bar{\rho}^{2} \bar{q}^{T}\left(\rho^{2} q-E\right)\right\rangle_{\mathrm{o}}+\vartheta \bar{\rho}\left\langle\left(\nabla_{y}(\bar{\rho} \bar{q})^{T} A_{0} \nabla_{y} q\right)\right\rangle_{\mathrm{o}}=0$. Учтем это соотношение в равенстве $\left\langle m_{0}\left(-E^{T}\right)\left(\rho^{2} q-E\right)\right\rangle_{\mathbf{o}}=-m$. Тогда

$$
-\rho m=\rho\left\langle m_{0}\left(\bar{\rho}^{2} \bar{q}-E\right)^{T}\left(\rho^{2} q-E\right)\right\rangle_{\mathbf{o}}+\vartheta \bar{\rho}\left\langle\left(\nabla_{y}(\bar{\rho} \bar{q})^{T} A_{0} \nabla_{y} \rho q\right)\right\rangle_{\mathbf{o}} .
$$

Непосредственно из этого равенства следует, что симметричная матрица

$$
\begin{aligned}
\operatorname{Re}(-\rho m) & =-\frac{1}{2}\left(\rho m+\overline{\rho m}^{T}\right) \\
& =\rho_{1}\left\langle m_{0}\left(\bar{\rho}^{2} \bar{q}-E\right)^{T}\left(\rho^{2} q-E\right)\right\rangle_{\mathbf{o}}+\vartheta \rho_{1}\left\langle\left(\nabla_{y}(\bar{\rho} \bar{q})^{T} A_{0}\left(\nabla_{y} \rho q\right)\right\rangle_{\mathbf{o}}\right.
\end{aligned}
$$

неотрицательно определена. Действительно, для $\rho \in \mathbb{C}_{0}$ и $\xi \in \mathbb{C}^{n}$ имеем

$$
\begin{aligned}
\bar{\xi}^{T} \operatorname{Re}(-\rho m) \xi= & \rho_{1}\left\langle m_{0}\left(\left(\bar{\rho}^{2} \bar{q}-E\right) \bar{\xi}\right)^{T}\left(\left(\rho^{2} q-E\right) \xi\right)\right\rangle_{\mathbf{o}} \\
& +\vartheta \rho_{1}\left\langle\nabla_{y}(\bar{\rho} \bar{q} \bar{\xi})^{T} A_{0} \nabla_{y}(\rho q \xi)\right\rangle_{\mathbf{o}} \\
\geqslant & \alpha \rho_{1}\left\|\left(\rho^{2} q-E\right) \xi\right\|_{L^{2}\left(Y_{0}\right)^{n}}^{2}+\alpha \vartheta \rho_{1}\left\|\nabla_{y}(\rho q \xi)\right\|_{L^{2}\left(Y_{0}\right)^{n \times n}} \geqslant 0
\end{aligned}
$$

Умножим скалярно в $\mathbb{C}^{n}$ первое уравнение из $(2.22)$ на $\bar{\rho} \bar{V}$ и проинтегрируем полученное соотношение по $\Omega$. Тогда

$$
\begin{aligned}
\rho \int_{\Omega}(M \rho V, \bar{\rho} \bar{V}) d x+\int_{\Omega}((-\rho m) \rho V, \bar{\rho} \bar{V}) d x+\bar{\rho} \int_{\Omega}(A \nabla V, \nabla \bar{V}) d x \\
=\int_{\Omega}\left(F_{0}, \bar{\rho} \bar{V}\right) d x-\int_{\Omega}(r F, \bar{\rho} \bar{V}) d x
\end{aligned}
$$


где $F_{0}=R F+\operatorname{div}(B G)$. Далее, рассмотрим уравнение, комплексно сопряженное к первому уравнению из (2.22). Умножая это уравнение на $\rho V$, интегрируя по $\Omega$ и складьвая полученное соотношение с (2.29), имеем

$$
\begin{array}{r}
\rho_{1} \int_{\Omega}(M \rho V, \bar{\rho} \bar{V}) d x+\int_{\Omega}(\operatorname{Re}(-\rho m) \rho V, \bar{\rho} \bar{V}) d x+\rho_{1} \int_{\Omega}(A \nabla V, \nabla \bar{V}) d x \\
=\operatorname{Re} \int_{\Omega}\left(F_{0}, \bar{\rho} \bar{V}\right) d x-\operatorname{Re} \int_{\Omega}(r F, \bar{\rho} \bar{V}) d x .
\end{array}
$$

Тогда из неравенства (2.28) следует, что

$$
\int_{\Omega}(M \rho V, \bar{\rho} \bar{V}) d x+\int_{\Omega}(A \nabla V, \nabla \bar{V}) d x \leqslant\left(\left\|F_{0}\right\|_{L^{2}(\Omega)^{n}}+\|r F\|_{L^{2}(\Omega)^{n}}\right)\|\rho V\|_{L^{2}(\Omega)^{n}}
$$

для всех $\rho=\rho_{1}+i \rho_{2}$ с $\rho_{1} \geqslant 1$. Поэтому, используя $\delta$-неравенство, (1.7), (2.27) и неравенство Корна, получаем

$$
\begin{aligned}
\|\rho V\|_{L^{2}(\Omega)^{n}}^{2}+\|\nabla V\|_{L^{2}(\Omega)^{n \times n}}^{2} & \leqslant C\left(\left\|F_{0}\right\|_{L^{2}(\Omega)^{n}}^{2}+\|r F\|_{L^{2}(\Omega)^{n}}^{2}\right) \\
& \leqslant C\left(\left\|F_{0}\right\|_{L^{2}(\Omega)^{n}}^{2}+\|F\|_{L^{2}(\Omega)^{n}}^{2}+\|\rho F\|_{L^{2}(\Omega)^{n}}^{2}\right)
\end{aligned}
$$

где постоянная $C$ не зависит от $\vartheta$ и $\rho \in \mathbb{C}_{1}$. Кроме того, умножая это неравенство на $|\rho|^{2 s}$ для $s=0,1, \ldots$, имеем априорную оценку

$$
\begin{aligned}
\left\|\rho^{s+1} V\right\|_{L^{2}(\Omega)^{n}}^{2} & +\left\|\rho^{s} \nabla V\right\|_{L^{2}(\Omega)^{n \times n}}^{2} \\
& \leqslant C\left(\left\|\rho^{s} F_{0}\right\|_{L^{2}(\Omega)^{n}}^{2}+\left\|\rho^{s} F\right\|_{L^{2}(\Omega)^{n}}^{2}+\left\|\rho^{s+1} F\right\|_{L^{2}(\Omega)^{n}}^{2}\right)
\end{aligned}
$$

где постоянная $C$ не зависит от $\vartheta, s$ и $\rho \in \mathbb{C}_{1}$.

Из неравенства (2.30) следует, что для каждого $\rho \in \mathbb{C}_{1}$ решение задачи $(2.22)$ существует и определено однозначно, поскольку система уравнений этой задачи является эллиптической системой уравнений с комплексными коэффициентами (см., например, [2]). Это решение является непрерывной функцией на $\mathbb{C}_{1}$ со значениями в $H_{0}^{1}(\Omega)^{n}$. Действительно, фиксируем несовпадающие $\rho$ и $\rho_{0}$ из $\mathbb{C}_{1}$. Тогда $V_{0}=V(\rho)-V\left(\rho_{0}\right)$ является решением краевой задачи

$$
\begin{gathered}
\rho^{2} M V_{0}-\rho^{2} m V_{0}-\operatorname{div}\left(A \nabla V_{0}\right)=\mathbb{F}(\rho)-\mathbb{F}\left(\rho_{0}\right)+\left(\mathbb{M}(\rho)-\mathbb{M}\left(\rho_{0}\right)\right) V\left(\rho_{0}\right) \text { в } \Omega \\
V_{0}=0 \text { на } \partial \Omega
\end{gathered}
$$

где $\mathbb{F}=F_{0}-r F$ и $\mathbb{M}=\rho^{2} M-m \rho^{2}$ являются голоморфными (и потому непрерывньпи) функциями на $\mathbb{C}_{0}$ со значениями в соответствующих пространствах. Следовательно, используя неравенство (2.30), имеем

$$
\left\|V(\rho)-V\left(\rho_{0}\right)\right\|_{H_{0}^{1}(\Omega)^{n}} \rightarrow 0 \text { при } \rho_{0} \rightarrow \rho,
$$

что доказывает непрерывность решения задачи (2.22).

Продифференцируем формально (2.22) по $\rho$ и рассмотрим краевую задачу

$$
\rho^{2} M V_{1}-\rho^{2} m V_{1}-\operatorname{div}\left(A \nabla V_{1}\right)=\mathbb{F}_{\rho}^{\prime}+\mathbb{M}_{\rho}^{\prime} V \text { в } \Omega, \quad V_{1}=0 \text { на } \partial \Omega .
$$


В силу уже доказанных результатов решение этой задачи сушествует, непрерьвно зависит от $\rho \in \mathbb{C}_{1}$ и удовлетворяет неравенству, аналогичному (2.30).

Разделим уравнения (2.31) на $\rho-\rho_{0}$ и вычтем из полученных соотношений уравнения (2.33). Тогда $V_{2}=\left(V(\rho)-V\left(\rho_{0}\right)\right) /\left(\rho-\rho_{0}\right)-V_{1}$ является решением краевой задачи

$$
\begin{aligned}
\rho^{2} M V_{2}-\rho^{2} m V_{2}-\operatorname{div}\left(A \nabla V_{2}\right) & =\mathbb{F}_{\Delta}-\mathbb{F}_{\rho}^{\prime}+\mathbb{M}_{\Delta} V_{0}+\left(\mathbb{M}_{\Delta}-\mathbb{M}_{\rho}^{\prime}\right) V \text { в } \Omega, \\
V_{2} & =0 \text { на } \partial \Omega,
\end{aligned}
$$

где $\mathbb{F}_{\Delta}=\left(\mathbb{F}(\rho)-\mathbb{F}\left(\rho_{0}\right)\right) /\left(\rho-\rho_{0}\right)$ и $\mathbb{M}_{\Delta}=\left(\mathbb{M}(\rho)-\mathbb{M}\left(\rho_{0}\right)\right) /\left(\rho-\rho_{0}\right)$. Следовательно, используя неравенство (2.30) и (2.32), имеем

$$
\left\|\frac{V(\rho)-V\left(\rho_{0}\right)}{\rho-\rho_{0}}-V_{1}\right\|_{H_{0}^{1}(\Omega)^{n}} \rightarrow 0 \text { при } \rho_{0} \rightarrow \rho .
$$

Таким образом, решение задачи (2.22) является голоморфной функцией на $\mathbb{C}_{1}$ со значениями в $H_{0}^{1}(\Omega)^{n}$.

Из неравенства (2.30) и теоремы 9 следует, что

$$
\begin{aligned}
& \left\|v_{t}^{(s+1)}\right\|_{L_{1}^{2}\left(0, \infty ; L^{2}(\Omega)^{n}\right)}^{2}+\left\|\nabla v_{t}^{(s)}\right\|_{L_{1}^{2}\left(0, \infty ; L^{2}(\Omega)^{n \times n}\right)}^{2} \\
& \quad \leqslant C\left(\left\|f_{t}^{(s+1)}\right\|_{L_{1}^{2}\left(0, \infty ; L^{2}(\Omega)^{n}\right)}^{2}+\left\|f_{t}^{(s)}\right\|_{L_{1}^{2}\left(0, \infty ; L^{2}(\Omega)^{n}\right)}^{2}+\left\|\nabla g_{t}^{(s)}\right\|_{L_{1}^{2}\left(0, \infty ; L^{2}(\Omega)^{n \times n}\right)}^{2}\right)
\end{aligned}
$$

где постоянная $C$ не зависит от $\vartheta$ и $s=0,1, \ldots$ Весовая функция $e^{-t}$ из определения пространства $L_{1}^{2}\left(0, \infty ; L^{2}(\Omega)^{n}\right)$ отделена от нуля на конечном интервале $(0, T)$ и поэтому

$$
\begin{aligned}
\|v\|_{H^{s+1}\left(0, T ; L^{2}(\Omega)^{n}\right)}^{2}+ & \|v\|_{H^{s}\left(0, T ; H_{0}^{1}(\Omega)^{n}\right)}^{2} \\
& \leqslant C\left(\|f\|_{H^{s+1}\left(0, T ; L^{2}(\Omega)^{n}\right)}^{2}+\|g\|_{H^{s}\left(0, T ; H^{1}(\Omega)^{n}\right)}^{2}\right)
\end{aligned}
$$

где постоянная $C$ не зависит от $\vartheta$, но зависит от $T$.

Перепишем (2.21) в виде “эллиптической” краевой задачи

$$
-\operatorname{div}\left(\widehat{A}_{1} \nabla v\right)=U \text { в } \Omega \times(0, T), \quad v=0 \text { на } \partial \Omega \times(0, T) .
$$

Из теоремы 9, неравенств (2.30), (2.27) для $r(\rho)$ и $m(\rho)$ следует, что $U \in H^{s-2}\left(0, T ; L^{2}(\Omega)^{n}\right) \cap H^{s-3}\left(0, T ; H_{0}^{1}(\Omega)^{n}\right)$. Здесь целое $s$ достаточно велико и оценки соответствующих норм не зависят от $\vartheta$. Поэтому для решения задачи (2.35) имеем $v \in H^{s-2}\left(0, T ; H^{2}(\Omega)^{n}\right) \cap H^{s-3}\left(0, T ; H^{3}(\Omega)^{n}\right)$ в силу эллиптической регулярности (см., например, [2]). Продолжая по индукции, получаем $v \in H^{s}\left(0, T ; H^{s}(\Omega)^{n}\right)$, что доказьвает лемму 10 для задачи $(2.21)$.

В случае $Y_{1}=\varnothing$ задача (1.21) также принимает вид (2.21), где $v=v_{2}, v_{1}=0$, $\widehat{A}_{1}=0, \widehat{B}_{1}=\widetilde{B}_{2}$ и в остальных обозначениях следует заменить единицу на 2. Поэтому, повторяя проведенное доказательство, в этом случае заключаем, что решение задачи (2.21) сушествует и

$$
\|v\|_{H^{s+1}\left(0, T ; L^{2}(\Omega)^{n}\right)}^{2} \leqslant C\left(\|f\|_{H^{s+1}\left(0, T ; L^{2}(\Omega)^{n}\right)}^{2}+\|g\|_{H^{s}\left(0, T ; H^{1}(\Omega)^{n}\right)}^{2}\right),
$$


где постоянная $C$ не зависит от $\vartheta$. Кроме того, гладкость по $x$ решения задачи $(2.21)$ эквивалентна гладкости по $x$ правой части этой задачи, что доказывает лемму 10 в рассматриваемом случае.

Для доказательства леммы 10 в общем случае введем обозначения

$$
\begin{gathered}
v=\left(\begin{array}{l}
v_{1} \\
v_{2}
\end{array}\right), \quad M=\left(\begin{array}{cc}
M_{1} & 0 \\
0 & M_{2}
\end{array}\right), \quad M_{0}=\left(\begin{array}{cc}
M_{1}^{1} & M_{2}^{1} \\
M_{1}^{2} & M_{2}^{2}
\end{array}\right), \quad A=\left(\begin{array}{cc}
\widehat{A}_{1} & 0 \\
0 & 0
\end{array}\right), \\
a_{0}=\left(\begin{array}{ll}
a_{1}^{1} & a_{2}^{1} \\
a_{1}^{2} & a_{2}^{2}
\end{array}\right), \quad R=\left(\begin{array}{l}
R_{1} \\
R_{2}
\end{array}\right), \quad R_{0}=\left(\begin{array}{c}
R_{1}^{0} \\
R_{2}^{0}
\end{array}\right), \quad B=\left(\begin{array}{c}
\widehat{B}_{1} \\
\widetilde{B}_{2}
\end{array}\right) .
\end{gathered}
$$

Непосредственно из определений следует, что симметричная матрица $a_{0}$ неотрицательно определена. Действительно, для $\xi=\left(\xi_{1}, \xi_{2}\right)^{T} \in \mathbb{C}^{n} \times \mathbb{C}^{\widetilde{n}}$ имеем

$$
\begin{aligned}
\bar{\xi}^{T} a_{0} \xi & =\bar{\xi}_{1}^{T} a_{1}^{1} \xi_{1}+\bar{\xi}_{1}^{T} a_{2}^{1} \xi_{2}+\bar{\xi}_{2}^{T} a_{1}^{2} \xi_{1}+\bar{\xi}_{2}^{T} a_{2}^{2} \xi_{2} \\
& =\vartheta\left\langle\nabla_{y}\left(E_{1} \bar{\xi}_{1}+E_{2} \bar{\xi}_{2}\right)^{T} A_{0} \nabla_{y}\left(E_{1} \xi_{1}+E_{2} \xi_{2}\right)\right\rangle_{\mathbf{o}} \geqslant 0
\end{aligned}
$$

Аналогично доказывается неравенство (2.28) для $m=\widehat{M}_{0}$. Следовательно, применяя преобразование Лапласа к задаче (1.21), получим для $V=\widehat{v}$ краевую задачу вида (2.22) с дополнительньм слагаемым $a_{0} V$, но это слагаемое несущественно в априорных оценках в силу неравенства (2.36). Таким образом, комбинирование доказательств уже рассмотренных случаев завершает доказательство леммы 10.

На самом деле в $\S \S 4-10$ используется только гладкость конечного порядка решений осредненных задач (1.15), (1.21), и (1.22):

$$
\partial_{t}^{\varkappa} \partial_{x_{1}}^{\varkappa_{1}} \cdots \partial_{x_{n}}^{\varkappa_{n}} v_{0} \in C^{0}\left([0, T] ; L^{\infty}(\Omega)\right) \text { для } \varkappa+\varkappa_{1}+\cdots+\varkappa_{n} \leqslant 4
$$

где через $\partial_{t}, \partial_{x_{1}}, \ldots, \partial_{x_{n}}$ обозначены соответствующие частные производные и $v_{0}$ обозначает компоненты соответствующих решений. Достаточные для выполнения (2.37) условия на исходные данные для задач (1.15) и (1.22) можно вывести из результатов работ [3] и [6], а для задачи (1.21) эти условия выводятся из доказательства леммы 10 и теорем вложений. Но мы не будем останавливаться на формулировке этих громоздких предположений на $f, g, w_{0}, w_{1}$ и $\Omega$.

\section{§ 3. Построение начальных членов асимптотики}

Алгоритм построения асимптотики решений общих эллиптических краевых задач с быстроосциллируюшими коэффициентами подробно описан в работе [8]. После применения преобразования Лапласа к (1.4) эта задача перейдет в эллиптическую краевую задачу с комплексньм параметром. Поэтому для построения начальных членов асимптотики решений этой задачи можно воспользоваться алгоритмом из [8]. Для упрощения построений удобно рассматривать задачу (1.4) как начально-краевую задачу с условиями сопряжения.

В соответствии с определениями из $\S 1$ область $\Omega$ составлена из трех множеств $\Omega_{0}^{\varepsilon}, \Omega_{1}^{\varepsilon}$ и $\Omega_{2}^{\varepsilon}$, разделенных общими границами:

$$
S_{0}^{\varepsilon} \equiv \partial \Omega_{0}^{\varepsilon} \backslash \partial \Omega, \quad S_{1}^{\varepsilon} \equiv \partial \Omega_{1}^{\varepsilon} \backslash \partial \Omega, \quad S_{2}^{\varepsilon} \equiv \partial \Omega_{2}^{\varepsilon} \backslash \partial \Omega, \quad S_{0}^{\varepsilon}=S_{1}^{\varepsilon} \cup S_{2}^{\varepsilon}
$$


Поэтому [2], [4] задачу (1.4) можно переписать как начально-краевую задачу с условиями сопряжения:

$$
\begin{gathered}
m_{0 \varepsilon} u_{t t}^{\prime \prime}-\sigma \operatorname{div}\left(A_{0 \varepsilon} \nabla u+B_{0 \varepsilon} g\right)=r_{0 \varepsilon} f \quad \text { в } \Omega_{0}^{\varepsilon} \times(0, T), \\
m_{1 \varepsilon} u_{t t}^{\prime \prime}-\operatorname{div}\left(A_{1 \varepsilon} \nabla u+B_{1 \varepsilon} g\right)=r_{1 \varepsilon} f \quad \text { в } \Omega_{1}^{\varepsilon} \times(0, T), \\
m_{2 \varepsilon} u_{t t}^{\prime \prime}-\operatorname{div}\left(A_{2 \varepsilon} \nabla u+B_{2 \varepsilon} g\right)=r_{2 \varepsilon} f \quad \text { в } \Omega_{2}^{\varepsilon} \times(0, T), \\
\left.u\right|_{t=0}=w_{0} \text { в } \Omega,\left.\quad u_{t}^{\prime}\right|_{t=0}=w_{1} \text { в } \Omega, \quad u=0 \text { на } \partial \Omega \times(0, T), \\
\nu_{\varepsilon}\left(A_{1 \varepsilon} \nabla u+B_{1 \varepsilon} g\right)=\sigma \nu_{\varepsilon}\left(A_{0 \varepsilon} \nabla u+B_{0 \varepsilon} g\right) \text { на } S_{1}^{\varepsilon} \times(0, T), \\
\nu_{\varepsilon}\left(A_{2 \varepsilon} \nabla u+B_{2 \varepsilon} g\right)=\sigma \nu_{\varepsilon}\left(A_{0 \varepsilon} \nabla u+B_{0 \varepsilon} g\right) \text { на } S_{2}^{\varepsilon} \times(0, T)
\end{gathered}
$$

и $u \in H_{0}^{1}(\Omega)^{n}$ для всех $t \in[0, T]$, где $m_{0 \varepsilon}=m_{0}(x / \varepsilon), \ldots$ Последние два равенства в (3.2) означают непрерывность вектора нормальных напряжений на внутренних границах (поверхностях контакта рассматриваемых сред). В этих равенствах для $s=0,1,2$ след $\nu_{\varepsilon}\left(A_{s \varepsilon} \nabla u+B_{s \varepsilon} g\right)$ в точке $x_{0} \in S_{s}^{\varepsilon}$ определяется как предельное значение при $x \rightarrow x_{0}$ вектор-функций $\nu_{\varepsilon}\left(A_{s \varepsilon} \nabla u+B_{s \varepsilon} g\right)$ в точках $x \in \Omega_{s}^{\varepsilon}$. Здесь $\nu_{\varepsilon}$ - внутренняя (внешняя) нормаль для $S_{0}^{\varepsilon}\left(S_{1}^{\varepsilon} \cup S_{2}^{\varepsilon}\right)$.

Следует отметить, что переход от задачи (1.4) к задаче (3.2) корректен, например, если компоненты тензоров $A_{0}, A_{1}$ и $A_{2}$ являются постоянными или гладкими функциями. В общем случае формулировку задачи (3.2) следует уточнить, но произведенный переход и дальнейшие манипуляции этого параграффа можно рассматривать как формальные соображения, приводящие к построению начальных членов асимптотики решения задачи (1.4). В дальнейших параграффах для построенной здесь асимптотики доказываются оценки точности из теорем 1-8, в формулировках которых подразумевается, что задача (1.4) определяется в вариационной форме (1.10).

Введем обозначения $U=\widehat{u}, F=\widehat{f}, G=\widehat{g}$ для преобразований Лапласа от функций $u, f$ и $g$ из (3.2). Тогда, применяя преобразование Лапласа к (3.2), получаем для $U \in H_{0}^{1}(\Omega)^{n}$ краевую задачу с условиями сопряжения и комплексным параметром $\rho$ :

$$
\begin{gathered}
\rho^{2} m_{0 \varepsilon} U-\sigma \operatorname{div}\left(A_{0 \varepsilon} \nabla U+B_{0 \varepsilon} G\right)=m_{0 \varepsilon}\left(\rho w_{0}+w_{1}\right)+r_{0 \varepsilon} F \text { в } \Omega_{0}^{\varepsilon}, \\
\rho^{2} m_{s \varepsilon} U-\operatorname{div}\left(A_{s \varepsilon} \nabla U+B_{s \varepsilon} G\right)=m_{s \varepsilon}\left(\rho w_{0}+w_{1}\right)+r_{s \varepsilon} F \text { в } \Omega_{s}^{\varepsilon}, \\
\nu_{\varepsilon}\left(A_{s \varepsilon} \nabla U+B_{s \varepsilon} G\right)=\sigma \nu_{\varepsilon}\left(A_{0 \varepsilon} \nabla U+B_{0 \varepsilon} G\right) \text { на } S_{s}^{\varepsilon}, \quad s=1,2 .
\end{gathered}
$$

В соответствии с [8] для построения асимптотики решения задачи (3.3) прежде всего следует описать ядро (нуль-пространство) оператора старшего порядка этой задачи, рассматриваемого на 1-периодических вектор-функциях при $\varepsilon=1$. Это ядро образуют столбцы матричнозначных функций $E_{1}(y)$ и $E_{2}(y)$, удовлетворяющих (1.16) и (1.18), что проверяется непосредственной подстановкой. Поэтому [8] с вектор-функции

$$
E_{1}\left(\frac{x}{\varepsilon}\right) V_{1}(x)+E_{2}\left(\frac{x}{\varepsilon}\right) V_{2}(x)
$$

начинается асимптотическое разложение решения задачи $(3.3)$, где $V_{1}=\left(v_{1}^{1}, \ldots\right.$, $\left.v_{n}^{1}\right)^{T}$ и $V_{2}=\left(v_{1}^{2}, \ldots, v_{\widetilde{n}}^{2}\right)^{T}$ пока не определены. Кроме того, к вектор-функции $(3.4)$ полезно добавить неизвестную вектор-функцию $V_{0}(x / \varepsilon, x)$ с носителем на $\Omega_{0}^{\varepsilon}$, поскольку заранее не определено, какой из операторов имеет старший порядок в первом уравнении из (3.3). Здесь и далее запись $V_{0}(x / \varepsilon, x)$ означает, что $V_{0}(y, x)$ является 1-периодической функцией от $y$ со значениями в подходящих пространствах. 
Таким образом, начальные члены асимптотического разложения решений задачи (3.3) можно попытаться искать в виде

$$
\begin{aligned}
U_{a}=E_{1}\left(\frac{x}{\varepsilon}\right) & V_{1}(x)+E_{2}\left(\frac{x}{\varepsilon}\right) V_{2}(x)+V_{0}\left(\frac{x}{\varepsilon}, x\right) \\
& +\varepsilon U_{1}\left(\frac{x}{\varepsilon}, x\right)+\varepsilon U_{2}\left(\frac{x}{\varepsilon}, x\right)+\varepsilon^{2} W_{1}\left(\frac{x}{\varepsilon}, x\right)+\varepsilon^{2} W_{2}\left(\frac{x}{\varepsilon}, x\right),
\end{aligned}
$$

где по определению $V_{0}=0$ на $\Omega_{12}^{\varepsilon}, E_{1} V_{1}, U_{1}, W_{1}=0$ на $\Omega_{2}^{\varepsilon}$ и $E_{2} V_{2}, U_{2}, W_{2}=0$ на $\Omega_{1}^{\varepsilon}$. Следующие две известные формулы для функций вида $V(x / \varepsilon, x)$, зависящих от $\varepsilon$ специальным образом, часто используются в дальнейшем

$$
\begin{aligned}
\nabla\left(V\left(\frac{x}{\varepsilon}, x\right)\right) & =\left.\left(\varepsilon^{-1} \nabla_{y} V(y, x)+\nabla_{x} V(y, x)\right)\right|_{y=x / \varepsilon} \\
\operatorname{div}\left(V\left(\frac{x}{\varepsilon}, x\right)\right) & =\left.\left(\varepsilon^{-1} \operatorname{div}_{y} V(y, x)+\operatorname{div}_{x} V(y, x)\right)\right|_{y=x / \varepsilon}
\end{aligned}
$$

Подставим равенство (3.5) вместо $U$ во второе уравнение из (3.3) при $s=2$. Тогда, учитывая (3.6) и сохраняя только существенные для дальнейшего слагаемые, имеем

$$
\begin{aligned}
& \rho^{2} m_{2} E_{2} V_{2}-\varepsilon^{-2} \operatorname{div}_{y}\left(A_{2} \nabla_{y}\left(E_{2} V_{2}\right)\right)-\varepsilon^{-1} \operatorname{div}_{x}\left(A_{2} \nabla_{y}\left(E_{2} V_{2}\right)\right) \\
& \quad-\varepsilon^{-1} \operatorname{div}_{y}\left(A_{2} \nabla_{x}\left(E_{2} V_{2}\right)+B_{2} G\right)-\operatorname{div}_{x}\left(A_{2} \nabla_{x}\left(E_{2} V_{2}\right)+B_{2} G\right) \\
& \quad-\varepsilon^{-1} \operatorname{div}_{y}\left(A_{2} \nabla_{y} U_{2}\right)-\operatorname{div}_{x}\left(A_{2} \nabla_{y} U_{2}\right)-\operatorname{div}_{y}\left(A_{2} \nabla_{x} U_{2}\right) \\
& \quad-\operatorname{div}_{y}\left(A_{2} \nabla_{y} W_{2}\right)=m_{2}\left(\rho w_{0}+w_{1}\right)+r_{2} F \text { при } y=x / \varepsilon \quad \text { и } \quad x \in \Omega_{2}^{\varepsilon} .
\end{aligned}
$$

Вектор-функция $E_{2}(y) V_{2}(x)$ является суммой столбцов матрицы $E_{2}(y)$, умноженных на компоненты вектора $V_{2}(x)$. Поэтому $A_{2} \nabla_{y}\left(E_{2} V_{2}\right)=A_{2} e_{y}\left(E_{2} V_{2}\right)=0$ в силу определений (см. (2.2) и (2.14)). Таким образом, еше два слагаемых в (3.7) являются несущественными.

1. Пусть выполнено условие (1.2). Тогда разность $\vartheta-\sigma / \varepsilon^{2}$ можно считать мальм параметром. Подставляя равенство (3.5) вместо $U$ в условия сопряжения из (3.3) при $s=2$, имеем

$$
\begin{aligned}
\nu_{\varepsilon}( & \left.A_{2} \nabla_{x}\left(E_{2} V_{2}\right)+B_{2} G+A_{2} \nabla_{y} U_{2}+\varepsilon A_{2} \nabla_{x} U_{2}+\varepsilon A_{2} \nabla_{y} W_{2}\right) \\
= & \nu_{\varepsilon}\left(\varepsilon \vartheta A_{0} \nabla_{y} V_{0}+\varepsilon \vartheta A_{0} \nabla_{y}\left(E_{1} V_{1}\right)+\varepsilon \vartheta A_{0} \nabla_{y}\left(E_{2} V_{2}\right)\right. \\
& \left.-\varepsilon\left(\vartheta-\frac{\sigma}{\varepsilon^{2}}\right)\left(A_{0} \nabla_{y} V_{0}+\cdots\right)\right) \text { при } y=x / \varepsilon \quad \text { и } x \in S_{2}^{\varepsilon} .
\end{aligned}
$$

Приравнивая коэффициенты при $\varepsilon^{-1}$ в (3.7) и при $\varepsilon^{0}$ в $(3.8)$, получаем

$$
\begin{aligned}
-\operatorname{div}_{y}\left(A_{2} \nabla_{y} U_{2}\right) & =\operatorname{div}_{y}\left(A_{2} E_{2} \nabla_{x} V_{2}+B_{2} G\right) \text { при } y=x / \varepsilon \quad \text { и } x \in \Omega_{2}^{\varepsilon}, \\
-\nu_{\varepsilon}\left(A_{2} \nabla_{y} U_{2}\right) & =\nu_{\varepsilon}\left(A_{2} E_{2} \nabla_{x} V_{2}+B_{2} G\right) \text { при } y=x / \varepsilon \quad \text { и } x \in S_{2}^{\varepsilon} .
\end{aligned}
$$

Эти равенства будут вьполнены, если выбрать $U_{2}=N_{2 a}(y) \nabla_{x} V_{2}(x)+N_{2 b}(y) G(x)$ и определить $N_{2 a}(y), N_{2 b}(y)$ как 1-периодические решения задач

$$
\begin{gathered}
-\operatorname{div}_{y}\left(A_{2} \nabla_{y} N_{2 a}\right)=\operatorname{div}_{y}\left(A_{2} E_{2}\right) \text { в } Y_{2}, \quad-\nu\left(A_{2} \nabla_{y} N_{2 a}\right)=\nu\left(A_{2} E_{2}\right) \text { на } S_{2}, \\
-\operatorname{div}_{y}\left(A_{2} \nabla_{y} N_{2 b}\right)=\operatorname{div}_{y} B_{2} \text { в } Y_{2}, \quad-\nu\left(A_{2} \nabla_{y} N_{2 b}\right)=\nu B_{2} \text { на } S_{2} .
\end{gathered}
$$


В координатной записи вторая из этих задач выписана в (1.13), а первая имеет вид: $(n \times \widetilde{n})$-матричнозначные функции $N_{2 a}^{p}(y), p=1, \ldots, n$, являются 1-периодическими решениями задач Неймана на $Y_{2}$ :

$$
\begin{aligned}
-\frac{\partial}{\partial y_{h}}\left(A_{2}^{h k} \frac{\partial}{\partial y_{k}} N_{2 a}^{p}\right) & =\frac{\partial}{\partial y_{h}}\left(A_{2}^{h p} E_{2}\right) \text { в } Y_{2}, \\
-\nu_{h}\left(A_{2}^{h k} \frac{\partial}{\partial y_{k}} N_{2 a}^{p}\right) & =\nu_{h}\left(A_{2}^{h p} E_{2}\right) \text { на } S_{2} .
\end{aligned}
$$

В соответствии с леммой 3 решения задач (3.9) однозначно определены и имеют компоненты из $H_{P}^{1}\left(Y_{2}\right)$. (Неявно здесь использовано равенство, аналогичное (2.14), которое следует из свойств симметрии (1.7).) Поэтому определены следы на $S_{2}$ этих компонент как элементы $H_{P}^{1 / 2}\left(S_{2}\right)$. Продолжим $N_{2 a}$ и $N_{2 b}$ на $Y_{0} \cup Y_{1}$ как 1-периодические решения задач Дирихле

$$
\begin{array}{llllllll}
\operatorname{div}_{y}\left(A_{0} \nabla_{y} N_{2 a}\right)=0 & \text { в } & Y_{0}, & N_{2 a}=0 & \text { на } & \bar{Y}_{1}, & N_{2 a}=N_{2 a} & \text { на } \bar{Y}_{2}, \\
\operatorname{div}_{y}\left(A_{0} \nabla_{y} N_{2 b}\right)=0 & \text { в } Y_{0}, & N_{2 b}=0 & \text { на } & \bar{Y}_{1}, & N_{2 b}=N_{2 b} & \text { на } \bar{Y}_{2} .
\end{array}
$$

Тогда $N_{2 a} \in H_{P}^{1}(Y)^{n \times \widetilde{n}}$ и $N_{2 b} \in H_{P}^{1}(Y)^{n \times n}$ (см., например, [2]).

Приравнивая коэффициенты при $\varepsilon^{0}$ в $(3.7)$ и при $\varepsilon^{1}$ в $(3.8)$, получаем

$$
\begin{aligned}
& -\operatorname{div}_{y}\left(A_{2} \nabla_{y} W_{2}\right)=r_{2} F+m_{2} E_{2}\left(\rho \widetilde{w}_{0}+\widetilde{w}_{1}\right)-\rho^{2} m_{2} E_{2} V_{2} \\
& +\operatorname{div}_{x}\left(\left(A_{2} E_{2}+A_{2} \nabla_{y} N_{2 a}\right) \nabla_{x} V_{2}+\left(B_{2}+A_{2} \nabla_{y} N_{2 b}\right) G\right) \\
& \quad+\operatorname{div}_{y}\left(A_{2} \nabla_{x} U_{2}\right) \text { при } y=x / \varepsilon \quad \text { и } x \in \Omega_{2}^{\varepsilon}, \\
& -\nu_{\varepsilon}\left(A_{2} \nabla_{y} W_{2}\right)=-\vartheta \nu_{\varepsilon}\left(A_{0} \nabla_{y} V_{0}+A_{0} \nabla_{y} E_{1} V_{1}+A_{0} \nabla_{y} E_{2} V_{2}\right) \\
& +\nu_{\varepsilon}\left(A_{2} \nabla_{x} U_{2}\right) \text { при } y=x / \varepsilon \text { и } x \in S_{2}^{\varepsilon},
\end{aligned}
$$

где учтены уже полученное представление для $U_{2}$ и определения $\widetilde{w}_{0}, \widetilde{w}_{1}$, данные перед (1.21). Рассмотрим эти соотношения как задачу Неймана для $W_{2}$ с параметром $x$ (подробности см., например, в [8]). В силу леммы 3 эта задача разрешима, если выполнено равенство

$$
\begin{aligned}
R_{2} F & +M_{2}\left(\rho \widetilde{w}_{0}+\widetilde{w}_{1}-\rho^{2} V_{2}\right) \\
+ & \operatorname{div}_{x}\left(\left\langle E_{2}^{T}\left(A_{2} E_{2}+A_{2} \nabla_{y} N_{2 a}\right)\right\rangle_{2} \nabla_{x} V_{2}+\widetilde{B}_{2} G\right) \\
& =-\vartheta \int_{S_{2}} E_{2}^{T} \nu\left(A_{0} \nabla_{y} V_{0}\right) d s-\vartheta \int_{S_{2}} E_{2}^{T} \nu\left(A_{0} \nabla_{y} E_{1} V_{1}+A_{0} \nabla_{y} E_{2} V_{2}\right) d s
\end{aligned}
$$

где учтены условия нормировки (1.17) и обозначения (1.20).

Поменяем в проведенных рассуждениях индексы $s=2$ и $s=1$ местами (см. $(3.7)-(3.13))$. Тогда $U_{1}=N_{1 a}(y) \nabla_{x} V_{1}(x)+N_{1 b}(y) G(x)$, где $N_{1 a}(y), N_{1 b}(y)$ являются решениями задач $(1.11),(1.13)$ на $Y_{1}$ и продолжены на $Y_{0} \cup Y_{2}$ как 1-периодические решения задач Дирихле, аналогичных (3.11). Кроме того, в силу леммы 2 условие разрешимости (3.13) следует заменить на равенство

$$
\begin{aligned}
R_{1} F & +M_{1}\left(\rho w_{0}+w_{1}-\rho^{2} V_{1}\right)+\operatorname{div}_{x}\left(\widehat{A}_{1} \nabla_{x} V_{1}+\widehat{B}_{1} G\right) \\
& =-\vartheta \int_{S_{1}} E_{1}^{T} \nu\left(A_{0} \nabla_{y} V_{0}\right) d s-\vartheta \int_{S_{1}} E_{1}^{T} \nu\left(A_{0} \nabla_{y} E_{1} V_{1}+A_{0} \nabla_{y} E_{2} V_{2}\right) d s
\end{aligned}
$$


где учтены обозначения (1.12), (1.20) и равенство $E_{1}=E$ на $\bar{Y}_{1}$ из (1.16).

В соответствии с определениями из $\S 1$ множество $Y_{2}$ отделено от границ ячейки $Y$, и поэтому условия 1-периодичности в задачах (3.9) выполнены автоматически. Следовательно, равенство $N_{2 a}^{p}=-y_{p} E_{2}$ определяет частное решение задачи (3.10). Действительно,

$$
A_{2}^{h k} \frac{\partial}{\partial y_{k}}\left(y_{p} E_{2}\right)=A_{2}^{h p} E_{2}+y_{p} A_{2}^{h k} \frac{\partial}{\partial y_{k}} E_{2}=A_{2}^{h p} E_{2}
$$

поскольку $A_{2} \nabla_{y} E_{2}=0$. Аналогично $N_{2 b}^{p}=-y_{p} E$, если $B_{2}=A_{2}$.

Таким образом, $\widehat{A}_{2}=\left\langle E_{2}^{T}\left(A_{2} E_{2}+A_{2} \nabla_{y} N_{2 a}\right)\right\rangle_{2}=0$ в (3.13). С другой стороны, тензор $\widehat{A}_{1}$ не является нулевьп, поскольку удовлетворяет условию эллиптичности (1.7), что следует из связности множества $F_{1}$ (см., например, [4], [5]).

Обозначим через $E_{2}^{1}(y)$ матрицу, составленную из первых $n$ столбцов матрицы $E_{2}(y)$. Тогда из $(1.16)$ и (1.18) непосредственно следует, что

$$
E_{1}(y)+E_{2}^{1}(y)=E \quad \text { для } \quad y \in Y
$$

Подставим равенство (3.5) вместо $U$ в первое уравнение из (3.3). Тогда, учитывая (1.16), (1.18), (3.6), (3.16) и сохраняя только существенные для дальнейшего слагаемые, имеем

$$
\begin{aligned}
\rho^{2} m_{0} V_{0}-\vartheta \operatorname{div}_{y} & \left(A_{0} \nabla_{y} V_{0}\right)=r_{0} F+m_{0} E_{1}\left(\rho w_{0}+w_{1}-\rho^{2} V_{1}\right) \\
& +m_{0} E_{2}\left(\rho \widetilde{w}_{0}+\widetilde{w}_{1}-\rho^{2} V_{2}\right) \text { при } y=x / \varepsilon \quad \text { и } x \in \Omega_{0}^{\varepsilon} .
\end{aligned}
$$

Напомним, что $\vartheta-\sigma / \varepsilon^{2}$ является малым параметром. Это равенство и равенство $V_{0}=0$ на $\Omega_{12}^{\varepsilon}$ будут выполнены, если выбрать

$$
V_{0}=\widehat{P} F-\widehat{Q}_{1}\left(\rho^{2} V_{1}-w_{1}-\rho w_{0}\right)-\widehat{Q}_{2}\left(\rho^{2} V_{2}-\widetilde{w}_{1}-\rho \widetilde{w}_{0}\right)
$$

и определить $\widehat{P}(\rho, y), \widehat{Q}_{1}(\rho, y), \widehat{Q}_{2}(\rho, y)$ как 1-периодические решения задач Дирихле на $Y_{0}$ с параметром $\rho$ :

$$
\begin{gathered}
m_{0}\left(\rho^{2} \widehat{P}-\frac{r_{0}}{m_{0}}\right)=\vartheta \operatorname{div}_{y}\left(A_{0} \nabla_{y} \widehat{P}\right) \text { в } Y_{0}, \quad \widehat{P}=0 \text { на } \bar{Y}_{12}, \\
m_{0}\left(\rho^{2} \widehat{Q}_{s}-E_{s}\right)=\vartheta \operatorname{div}_{y}\left(A_{0} \nabla_{y} \widehat{Q}_{s}\right) \text { в } Y_{0}, \quad \widehat{Q}_{s}=0 \text { на } \bar{Y}_{12}, \quad s=1,2 .
\end{gathered}
$$

Отметим, что обратное преобразование Лапласа от этих задач совпадает в точности с задачами (1.19).

Умножим второе уравнение из (3.19) на $E_{l}^{T}$ для $l=1,2$ и проинтегрируем полученное соотношение по $Y_{0}$. Тогда, учитывая $(1.16),(1.18)$ и $(2.10)$, имеем

$$
\begin{aligned}
\left\langle m_{0} E_{l}^{T}\left(\rho^{2} \widehat{Q}_{s}-E_{s}\right)\right\rangle_{\mathbf{o}} & =\vartheta\left\langle E_{l}^{T} \operatorname{div}_{y}\left(A_{0} \nabla_{y} \widehat{Q}_{s}\right)\right\rangle_{\mathbf{o}} \\
& =-\vartheta\left\langle\nabla_{y} E_{l}^{T} A_{0} \nabla_{y} \widehat{Q}_{s}\right\rangle_{\mathbf{o}}-\vartheta \int_{S_{0}} E_{l}^{T} \nu\left(A_{0} \nabla_{y} \widehat{Q}_{s}\right) d s \\
& =-\vartheta \int_{S_{l}} E_{l}^{T} \nu\left(A_{0} \nabla_{y} \widehat{Q}_{s}\right) d s
\end{aligned}
$$


С другой стороны, $\left\langle m_{0} E_{l}^{T}\left(\rho^{2} \widehat{Q}_{s}-E_{s}\right)\right\rangle_{\text {o }}$ совпадает с преобразованием Лапласа от матричнозначных функций $M_{s}^{l}(t)$, определенных в $(1.20)$ для $s, l=1,2$. Кроме того, умножая первое уравнение из (3.19) на $E_{l}^{T}$ для $l=1,2$, получим представление через граничные интегралы для преобразования Лапласа от матричнозначных функций $R_{1}^{0}(t)$ и $R_{2}^{0}(t)$ из (1.20). Аналогично, умножая уравнения из $(1.16),(1.18)$ на $E_{l}^{T}$ для $l=1,2$, имеем

$$
a_{s}^{l}=\vartheta\left\langle\nabla_{y} E_{l}^{T} A_{0} \nabla_{y} E_{s}\right\rangle_{\mathbf{o}}=-\vartheta \int_{S_{l}} E_{l}^{T} \nu\left(A_{0} \nabla_{y} E_{s}\right) d s, \quad l, s=1,2 .
$$

Обозначим через $v_{1}, v_{2}$ обратные преобразования Лапласа от $V_{1}$ и $V_{2}$ соответственно. Подставим соотношение (3.18) вместо $V_{0}$ в равенства (3.13) и (3.14). Тогда, применяя обратное преобразование Лапласа к этим равенствам и учитывая $(3.20),(3.21)$, получим для $v_{1}, v_{2}$ начально-краевую задачу (1.21). Кроме того, из (3.5) имеем представление для начальных членов асимптотического разложения решения задачи (1.4):

$$
\begin{aligned}
u_{a}=E_{1 \varepsilon} v_{1}+E_{2 \varepsilon} v_{2}+ & P_{\varepsilon} * f-Q_{1 \varepsilon} *\left(\left(v_{1}\right)_{t t}^{\prime \prime}\right)-Q_{2 \varepsilon} *\left(\left(v_{2}\right)_{t t}^{\prime \prime}\right) \\
& +\varepsilon N_{1 a \varepsilon} \nabla_{x} v_{1}+\varepsilon N_{1 b \varepsilon} g+\varepsilon N_{2 a \varepsilon} \nabla_{x} v_{2}+\varepsilon N_{2 b \varepsilon} g .
\end{aligned}
$$

Следуя [8], можно определить $W_{2}$ из (3.12) и продолжить построение асимптотического разложения, но представления (3.22) будет достаточно для доказательства оценки точности из теоремы 2. Кроме того, дальнейшие построения асимптотики не приводят к сушественному улучшению этой оценки, что связано с краевыми эффектами, возникающими в задаче (1.4) вблизи $\partial \Omega$.

2. Пусть выполнено условие (1.3). Тогда $\omega=\sqrt{\sigma} / \varepsilon$ можно считать малым параметром. Повторим проведенные в этом параграфе построения в случае, когда $\vartheta=\omega^{2}$ (см. (3.7)-(3.22)). Тогда для начальных членов асимптотического разложения решения задачи (1.4) получим представление (3.22). В этом представлении $v_{1}, v_{2}$ являются решением начально-краевой задачи (1.21) и $P, Q_{1}, Q_{2}$ удовлетворяют задачам (1.19). Эти задачи зависят от малого параметра $\omega$, что приводит к некоторому упрощению представления (3.22).

В соответствии с (3.21) коэффициенты $a_{s}^{l}$ имеют порядок $\omega^{2}$, и поэтому можно положить $a_{s}^{l}=0$ в (1.21) для $s, l=1,2$. Используя определения (1.20) и формулы дифференцирования сверток (2.20), получаем

$$
\begin{aligned}
R_{s}^{0} * f & =\left\langle m_{0} E_{s}^{T} P_{t t}^{\prime \prime}\right\rangle_{\mathbf{o}} * f=\left(\left\langle m_{0} E_{s}^{T} \int_{0}^{t} P_{t t}^{\prime \prime} d t\right\rangle_{\mathbf{o}}\right)_{t}^{\prime} * f \\
& =\left\langle m_{0} E_{s}^{T}\left(P_{t}^{\prime}-\frac{r_{0}}{m_{0}} E\right)\right\rangle_{\mathbf{o}} *\left(f_{t}^{\prime}\right)+\left\langle m_{0} E_{s}^{T}\left(P_{t}^{\prime}-\frac{r_{0}}{m_{0}} E\right)\right\rangle_{\mathbf{o}}\left(\left.f\right|_{t=0}\right) .
\end{aligned}
$$

Из лемм 6, 8 и неравенства Коши-Буняковского следует, что эти величины имеют порядок $\sqrt{\omega}$, и поэтому полагаем $R_{s}^{0}=0$ в $(1.21)$ для $s=1,2$. По аналогичной причине выбираем $M_{s}^{l}=0$ в (1.21) для $s, l=1,2$. Тогда $v_{1}, v_{2}$ являются решениями начально-краевых задач (1.22).

Вновь используя формулы дифференцирования (2.20), имеем

$$
Q_{1 \varepsilon} *\left(v_{1}^{\prime \prime}\right)=\left(Q_{1 \varepsilon} *\left(\int_{0}^{t} v_{1}^{\prime \prime} d t\right)\right)_{t}^{\prime}=\left(Q_{1 \varepsilon}\right)_{t}^{\prime} *\left(v_{1}^{\prime}-w_{1}\right) .
$$


Здесь и далее $v_{1}^{\prime}=\left(v_{1}\right)_{t}^{\prime}$ и $v_{1}^{\prime \prime}=\left(v_{1}\right)_{t t}^{\prime \prime}$. В силу леммы 6 разность $\left(Q_{1 t}^{\prime}-E_{1}\right)_{\varepsilon}$ имеет порядок $\sqrt{\omega}$. Поэтому из леммы 7 следует асимптотическое равенство

$$
Q_{1 \varepsilon} *\left(v_{1}^{\prime \prime}\right)=E_{1 \varepsilon} \int_{0}^{t}\left(v_{1}^{\prime}-w_{1}\right) d t=E_{1 \varepsilon}\left(v_{1}-w_{1} t-w_{0}\right)
$$

поскольку свертка с функцией $E_{1 \varepsilon}$, не зависящей от $t$, совпадает с взятием первообразной (см. (2.19)). Аналогичные асимптотические равенства выполнены для $Q_{2 \varepsilon} *\left(v_{2}^{\prime \prime}\right)$ и $P_{\varepsilon} * f$. Напомним также, что по определению $P_{\varepsilon}, Q_{1 \varepsilon}, Q_{2 \varepsilon}=0$ на $\bar{\Omega}_{12}^{\varepsilon}$, $E_{1 \varepsilon}=0$ на $\bar{\Omega}_{2}^{\varepsilon}, E_{2 \varepsilon}=0$ на $\bar{\Omega}_{1}^{\varepsilon}$. Таким образом, представление (3.22) в рассматриваемом случае можно переписать в виде

$$
u_{a}=\chi_{1}^{\varepsilon} E_{1 \varepsilon} v_{1}+\chi_{2}^{\varepsilon} E_{2 \varepsilon} v_{2}+\chi_{0}^{\varepsilon}\left(w_{0}+w_{1} t+\left(\frac{r_{0}}{m_{0}}\right)_{\varepsilon} \int_{0}^{t} \int_{0}^{t} f d t d t\right)+\varepsilon u_{1},
$$

где $\chi_{0}^{\varepsilon}, \chi_{1}^{\varepsilon}$ и $\chi_{2}^{\varepsilon}$ обозначают характеристические функции множеств $\Omega_{0}^{\varepsilon}, \Omega_{1}^{\varepsilon}$ и $\Omega_{2}^{\varepsilon}$ соответственно. Здесь и далее $u_{1}=N_{1 a \varepsilon} \nabla_{x} v_{1}+N_{1 b \varepsilon} g+N_{2 a \varepsilon} \nabla_{x} v_{2}+N_{2 b \varepsilon} g$.

3. Пусть выполнено условие (1.1). Тогда $\omega=\varepsilon^{2} / \sigma$ можно считать малым параметром. Повторим проведенные в п. 1 этого параграффа построения в случае, когда $\vartheta=1 / \omega$ (см. (3.7)-(3.21)). Тогда для начальных членов асимптотического разложения решения задачи (1.4) получим представление (3.22). Используя формулы (2.20), имеем

$$
P_{\varepsilon} * f=\left(\left(\int_{0}^{t} P_{\varepsilon} d t\right) * f\right)_{t}^{\prime}=\dot{P}_{\varepsilon} *\left(f_{t}^{\prime}\right)+\dot{P}_{\varepsilon}\left(\left.f\right|_{t=0}\right) .
$$

Поэтому из лемм 5 и 7 следует асимптотическое равенство

$$
P_{\varepsilon} * f=\frac{\varepsilon^{2}}{\sigma} p_{\varepsilon}\left(\int_{0}^{t} f_{t}^{\prime} d t\right)+\frac{\varepsilon^{2}}{\sigma} p_{\varepsilon}\left(\left.f\right|_{t=0}\right)=\frac{\varepsilon^{2}}{\sigma} p_{\varepsilon} f .
$$

Аналогичные равенства выполнены для $Q_{1 \varepsilon} *\left(v_{1}^{\prime \prime}\right)$ и $Q_{2 \varepsilon} *\left(v_{2}^{\prime \prime}\right)$. Таким образом, представление (3.22) в рассматриваемом случае можно переписать в виде

$$
u_{a}=E_{1 \varepsilon} v_{1}+E_{2 \varepsilon} v_{2}+\frac{\varepsilon^{2}}{\sigma} p_{\varepsilon} f-\frac{\varepsilon^{2}}{\sigma} q_{1 \varepsilon}\left(v_{1}^{\prime \prime}\right)-\frac{\varepsilon^{2}}{\sigma} q_{2 \varepsilon}\left(v_{2}^{\prime \prime}\right)+\varepsilon u_{1},
$$

где $p, q_{1}$ и $q_{2}$ являются 1-периодическими решениями задач (2.15). Следовательно, выражение (3.18) для $V_{0}$ можно записать в виде

$$
V_{0}=\frac{\varepsilon^{2}}{\sigma}\left(p F-q_{1}\left(\rho^{2} V_{1}-w_{1}-\rho w_{0}\right)-q_{2}\left(\rho^{2} V_{2}-\widetilde{w}_{1}-\rho \widetilde{w}_{0}\right)\right) .
$$

Умножим второе уравнение из $(2.15)$ на $E_{l}^{T}$ для $l=1,2$ и проинтегрируем полученное соотношение по $Y_{0}$. Тогда, учитывая $(1.16),(1.18)$ и $(2.10)$, имеем

$$
\begin{aligned}
\widehat{m}_{s}^{l} & \equiv\left\langle m_{0} E_{l}^{T} E_{s}\right\rangle_{\mathbf{o}}=-\left\langle E_{l}^{T} \operatorname{div}_{y}\left(A_{0} \nabla_{y} q_{s}\right)\right\rangle_{\mathbf{o}} \\
& =\left\langle\nabla_{y} E_{l}^{T} A_{0} \nabla_{y} q_{s}\right\rangle_{\mathbf{o}}+\int_{S_{0}} E_{l}^{T} \nu\left(A_{0} \nabla_{y} q_{s}\right) d s=\int_{S_{l}} E_{l}^{T} \nu\left(A_{0} \nabla_{y} q_{s}\right) d s
\end{aligned}
$$


для $s, l=1,2$ (ср. с (3.20)). Аналогично, умножая первое уравнение из (2.15) на $E_{l}^{T}$ для $l=1,2$, получаем

$$
\widehat{r}_{l}^{0} \equiv\left\langle r_{0} E_{l}^{T} E\right\rangle_{\mathrm{o}}=\int_{S_{l}} E_{l}^{T} \nu\left(A_{0} \nabla_{y} p\right) d s .
$$

Подставим соотношение (3.28) вместо $V_{0}$ в равенства (3.13) и (3.14). Тогда, применяя обратное преобразование Лапласа к этим равенствам и учитывая (3.21), $(3.29),(3.30)$, получим для $v_{1}, v_{2}$ начально-краевую задачу

$$
\begin{gathered}
M_{1} v_{1}^{\prime \prime}+\widehat{m}_{1}^{1} v_{1}^{\prime \prime}+\widehat{m}_{2}^{1} v_{2}^{\prime \prime}-\operatorname{div}\left(\widehat{A}_{1} \nabla v_{1}\right)+a_{1}^{1} v_{1}+a_{2}^{1} v_{2} \\
=R_{1} f+\widehat{r}_{1}^{0} f+\operatorname{div}\left(\widehat{B}_{1} g\right) \text { в } \Omega \times(0, T), \\
M_{2} v_{2}^{\prime \prime}+\widehat{m}_{1}^{2} v_{1}^{\prime \prime}+\widehat{m}_{2}^{2} v_{2}^{\prime \prime}+a_{1}^{2} v_{1}+a_{2}^{2} v_{2} \\
=R_{2} f+\widehat{r}_{2}^{0} f+\operatorname{div}\left(\widetilde{B}_{2} g\right) \text { в } \Omega \times(0, T), \\
\left.v_{1}\right|_{t=0}=w_{0} \text { в } \Omega,\left.\quad v_{1}^{\prime}\right|_{t=0}=w_{1} \text { в } \Omega, \quad v_{1}=0 \text { на } \partial \Omega \times(0, T), \\
\left.v_{2}\right|_{t=0}=\widetilde{w}_{0} \text { в } \Omega,\left.\quad v_{2}^{\prime}\right|_{t=0}=\widetilde{w}_{1} \text { в } \Omega .
\end{gathered}
$$

Отметим, что при переходе к задаче (3.31) сингулярньй множитель $\vartheta=\sigma / \varepsilon^{2}$ при граничных интегралах из (3.13), (3.14), содержащих $V_{0}$, сокращается с соответствующим множителем из (3.28), но остается в коэффициентах матриц $a_{s}^{l}$ для $s, l=1,2$ (см. (3.21)). Введем обозначения

$$
\widetilde{a}=\frac{\varepsilon^{2}}{\sigma}\left(\begin{array}{ll}
a_{1}^{1} & a_{2}^{1} \\
a_{1}^{2} & a_{2}^{2}
\end{array}\right)=\frac{1}{\vartheta}\left(\begin{array}{ll}
a_{1}^{1} & a_{2}^{1} \\
a_{1}^{2} & a_{2}^{2}
\end{array}\right), \quad \widetilde{m}=\left(\begin{array}{ll}
\widehat{m}_{1}^{1} & \widehat{m}_{2}^{1} \\
\widehat{m}_{1}^{2} & \widehat{m}_{2}^{2}
\end{array}\right) .
$$

Непосредственно из определений следует, что симметричные матрицы $a_{0}, \widetilde{a}, \widetilde{m}$ неотрицательно определены (см. доказательство неравенства (2.36)). Следовательно, повторяя доказательство леммы 10, получаем следующее утверждение.

Лемма 11. Пусть $f, g_{k} \in C_{0}^{\infty}((0, T) \times \Omega)^{n}, w_{0}=0 u w_{1}=0$, 2де $k=$ $1, \ldots, n$. Тогда существует единственное достаточно гладкое решение начально-краевой задачи (3.31), причем константы в оценках соответствующих норм не зависят от $\sigma / \varepsilon^{2}$.

Будем рассматривать матрицу $\widetilde{a}$ как $(3 \times 3)$-матрицу, действуюшую на $\mathbb{R}^{n} \times$ $\mathbb{R}^{n} \times \mathbb{R}^{\widetilde{n}-n}$ и составленную из матричных блоков $\widetilde{a}_{s}^{l}, s, l=0,1,2$, соответствующих размеров. Симметричный матричный блок $a \equiv \widetilde{a}_{1}^{1}=\left\langle\nabla_{y} E_{1}^{T} A_{0} \nabla_{y} E_{1}\right\rangle_{\mathbf{o}}$ является "скалярным" квадратом матрицы $E_{1}$, составленной из линейно независимых столбцов. Следовательно, матрица $а$ положительно определена и обратима. Действительно, $\xi^{T} a \xi=\left\langle\nabla_{y}\left(E_{1} \xi\right)^{T} A_{0} \nabla_{y}\left(E_{1} \xi\right)\right\rangle_{\mathbf{o}} \geqslant 0$ для $\xi \in \mathbb{R}^{n}$. Пусть $\xi_{0}^{T} a \xi_{0}=0$ для некоторого $\xi_{0} \in \mathbb{R}^{n}$. Тогда $E_{1} \xi_{0}$ является жестким перемещением на $Y_{0}$. По определению $E_{1}=0$ на одной из частей границы $\partial Y_{0}$ и $E_{1}=E$ на другой части, поэтому $\xi_{0}=0$. Таким образом, матрица $a$ положительно определена.

Симметричный матричный блок $d \equiv \widetilde{a}_{3}^{3}=\left\langle\nabla_{y} E_{3}^{T} A_{0} \nabla_{y} E_{3}\right\rangle_{\text {o }}$ также является "скалярньм" квадратом матрицы $E_{3}$, составленной из последних (линейно независимых $) \widetilde{n}-n$ столбцов матрицы $E_{2}$. Следовательно, матрица $d$ положительно 
определена и обратима. По аналогичной причине обратим и “скалярный" квадрат матрицы $E_{2}$ : найдется такая матрица $c$ на $\mathbb{R}^{n} \times \mathbb{R}^{\widetilde{n}-n}$, что

$$
c\left(\begin{array}{cc}
\widetilde{a}_{2}^{2} & \widetilde{a}_{3}^{2} \\
\widetilde{a}_{2}^{3} & \widetilde{a}_{3}^{3}
\end{array}\right) \equiv\left(\begin{array}{cc}
c_{1} & c_{2} \\
c_{2}^{T} & c_{3}
\end{array}\right)\left(\begin{array}{cc}
\widetilde{a}_{2}^{2} & \widetilde{a}_{3}^{2} \\
\widetilde{a}_{2}^{3} & \widetilde{a}_{3}^{3}
\end{array}\right)=\left(\begin{array}{cc}
E & 0 \\
0 & E_{\widetilde{n}-n}
\end{array}\right),
$$

где $E_{\widetilde{n}-n}$ обозначает единичную матрищу для $\mathbb{R}^{\widetilde{n}-n}$.

Определим $b \equiv \widetilde{a}_{3}^{1}=\left\langle\nabla_{y} E_{1}^{T} A_{0} \nabla_{y} E_{3}\right\rangle_{\mathbf{o}}$ и напомним, что $a=\widetilde{a}_{1}^{1}, d=\widetilde{a}_{3}^{3}$. Из равенства (3.16) следует, что

$$
\nabla_{y} E_{1}=-\nabla_{y} E_{2}^{1}, \quad A_{0} \nabla_{y} E_{1}=-A_{0} \nabla_{y} E_{2}^{1} \quad \text { для } y \in Y_{0} .
$$

Учитывая эти равенства в определениях (3.21) и (3.32), имеем

$$
\begin{array}{lll}
\widetilde{a}_{1}^{1}=a, & \widetilde{a}_{2}^{1}=-a, & \widetilde{a}_{3}^{1}=b, \\
\widetilde{a}_{1}^{2}=-a, & \widetilde{a}_{2}^{2}=a, & \widetilde{a}_{3}^{2}=-b, \\
\widetilde{a}_{1}^{3}=b^{T}, & \widetilde{a}_{2}^{3}=-b^{T}, & \widetilde{a}_{3}^{3}=d .
\end{array}
$$

Обозначим через $D$ обратимую матрицу на $\mathbb{R}^{n} \times \mathbb{R}^{\widetilde{n}}$, диагональ которой составлена из блоков $a^{-1}$ и $c$, а остальные элементы нулевые. Тогда из (3.33) и (3.34) заключаем, что

$$
D \widetilde{a}=\left(\begin{array}{ccc}
a^{-1} & 0 & 0 \\
0 & c_{1} & c_{2} \\
0 & c_{2}^{T} & c_{3}
\end{array}\right)\left(\begin{array}{ccc}
a & -a & b \\
-a & a & -b \\
b^{T} & -b^{T} & d
\end{array}\right)=\left(\begin{array}{ccc}
E & -E & a^{-1} b \\
-E & E & 0 \\
0 & 0 & E_{\widetilde{n}-n}
\end{array}\right) .
$$

Пусть $v_{2}^{1}$ и $v_{3}$ обозначают первые $n$ и последние $\widetilde{n}-n$ компонент вектор-функции $v_{2}$ и $w=\left(v_{1}, v_{2}\right)^{T}=\left(v_{1}, v_{2}^{1}, v_{3}\right)^{T}$. Выделяя сингулярные слагаемые в первых двух уравнениях из (3.31), получаем

$$
\frac{\sigma}{\varepsilon^{2}} \widetilde{a} w=W .
$$

В силу леммы $11 W \in H^{s}\left(0, T ; H^{s}(\Omega)^{n+\widetilde{n}}\right)$ для $s=0,1, \ldots$, причем константы в оценках соответствуюших норм не зависят от $\sigma / \varepsilon^{2}$. Таким образом, учитывая $(3.35)$ и $(3.36)$, для решения $v_{1}, v_{2}$ системы уравнений (3.31) получаем неравенство

$$
\left\|v_{1}-v_{2}^{1}\right\|_{H^{s}\left(0, T ; H^{s}(\Omega)^{n}\right)}+\left\|v_{3}\right\|_{H^{s}\left(0, T ; H^{s}(\Omega)^{\tilde{n}}-n\right)} \leqslant C \frac{\varepsilon^{2}}{\sigma},
$$

где $s=0,1, \ldots$ и постоянная $C$ не зависит от $\sigma / \varepsilon^{2}$.

Учитьвая равенства (3.34), сложим первые $n$ и следующие $n$ уравнений в (3.36). Тогда сингулярные слагаемые сократятся и соответствуюшая сумма запишется в виде

$$
\begin{aligned}
M_{1} v_{1}^{\prime \prime}+ & \widehat{m}_{1}^{1} v_{1}^{\prime \prime}+\widehat{m}_{2}^{1} v_{2}^{\prime \prime}-\operatorname{div}\left(\widehat{A}_{1} \nabla v_{1}\right)+M_{2}\left(v_{2}^{1}\right)^{\prime \prime}+\widetilde{m}_{1}^{2} v_{1}^{\prime \prime}+\widetilde{m}_{2}^{2} v_{2}^{\prime \prime} \\
= & \left(R_{1}+\widetilde{R}_{2}\right) f+\left(\widehat{r}_{1}^{0}+\widehat{r}_{2}^{0}\right) f+\operatorname{div}\left(\left(\widehat{B}_{1}+\widehat{B}_{2}\right) g\right) \text { в } \Omega \times(0, T),
\end{aligned}
$$

где $\widetilde{R}_{2}=\left\langle\left(E_{2}^{1}\right)^{T} r_{2}\right\rangle_{2}, \widetilde{r}_{2}^{0}=\left\langle r_{0}\left(E_{2}^{1}\right)^{T} E\right\rangle_{\mathbf{0}}, \widetilde{m}_{1}^{2}=\left\langle m_{0}\left(E_{2}^{1}\right)^{T} E_{1}\right\rangle_{\mathbf{0}}$ и $\widetilde{m}_{2}^{2}=\left\langle m_{0}\left(E_{2}^{1}\right)^{T} E_{2}\right\rangle_{\mathbf{0}}$. Из равенства $(3.16)$ следует, что $\widehat{r}_{1}^{0}+\widetilde{r}_{2}^{0}=\left\langle r_{0}\right\rangle_{\mathbf{o}} E$. Поэтому $\left(R_{1}+\widetilde{R}_{2}+\left\langle r_{0}\right\rangle_{\mathbf{o}}\right) f=R f$ 
в соответствии с $(1.14),(1.16),(1.18)$ и (1.20). Используя неравенство (3.37), можно заменить с достаточной степенњю точности $v_{2}$ и $v_{2}^{1}$ на $v_{1}$ в соотношении (3.38). Тогда $v=v_{1}$ является решением начально-краевой задачи $(1.15)$, поскольку соответствующие коэффициенты $\widehat{m}_{1}^{1}, \widehat{m}_{2}^{1}, \widetilde{m}_{1}^{2}, \widetilde{m}_{2}^{2}$ в сумме равны $\left\langle m_{0}\right\rangle_{\mathbf{o}} E$.

Решение задачи (1.15) определено однозначно и поэтому с достаточной степенњ точности приближает решение задачи (3.31) (в смысле выполнения неравенства (3.37)). Таким образом, представление (3.27) для начальных членов асимптотики в рассматриваемом случае можно переписать в виде

$$
u_{a}=v+E_{2 \varepsilon}^{1}\left(v_{2}^{1}-v\right)+E_{3 \varepsilon} v_{3}+\frac{\varepsilon^{2}}{\sigma} p_{\varepsilon} f-\frac{\varepsilon^{2}}{\sigma} q_{1 \varepsilon} v_{1}^{\prime \prime}-\frac{\varepsilon^{2}}{\sigma} q_{2 \varepsilon} v_{2}^{\prime \prime}+\varepsilon u_{1},
$$

где второе и третье слагаемые в правой части этого равенства достаточно малы по крайней мере в норме пространства $H^{s}\left(0, T ; L^{2}(\Omega)^{n}\right)$ для $s=0,1, \ldots$ Для доказательства теоремы 1 при однородных начальных условиях достаточно воспользоваться разложением (3.39). Однако эти условия не являются естественными в рассматриваемом случае. Поэтому удобней “переразложить" представление (3.39) для начальных членов асимптотического разложения.

В соответствии с (3.37) определим “новое" $v_{2}$ равенством $\left(\varepsilon^{2} / \sigma\right) v_{2}=\left(v_{2}^{1}-v, v_{3}\right)$. Учтем также (3.16) и (3.37) в остальных слагаемых из (3.39) и представим $u_{a}$ в виде

$$
u_{a}=v+\frac{\varepsilon^{2}}{\sigma} E_{2 \varepsilon} v_{2}+\frac{\varepsilon^{2}}{\sigma} p_{\varepsilon} f-\frac{\varepsilon^{2}}{\sigma} q_{\varepsilon} v_{t t}^{\prime \prime}+\varepsilon U_{1}+\varepsilon U_{2}+\varepsilon^{2} W_{1}+\varepsilon^{2} W_{2},
$$

где $U_{1}, U_{2}, W_{1}$ и $W_{2}$ определяются так же, как и в (3.5). Kроме того, $q \in H_{P}^{1}(Y)^{n \times n}$ является 1-периодическим решением задачи Дирихле

$$
-\operatorname{div}_{y}\left(A_{0} \nabla_{y} q\right)=m_{0} E \quad \text { в } Y_{0}, \quad q=0 \text { на } \bar{Y}_{12} .
$$

Заметим, что $q=q_{1}+q_{2}^{1}$ в соответствии с (2.15) и (3.16), где $q_{2}^{1}$ обозначает матрицу, составленную из первых $n$ столбцов матрицы $q_{2}$.

Подставим равенство (3.40) вместо $u$ в третье и последнее уравнения из (3.2). Тогда (не используя преобразование Лапласа) получим соотношения, аналогичные (3.7)-(3.12). Учитывая (3.40), наиболее существенное изменение следует внести в (3.8):

$$
\begin{aligned}
\nu_{\varepsilon}\left(A_{2} \nabla_{x} v+B_{2} g+A_{2}\right. & \left.\nabla_{y} U_{2}+\varepsilon A_{2} \nabla_{x} U_{2}+\varepsilon A_{2} \nabla_{y} W_{2}\right) \\
& =\nu_{\varepsilon}\left(\varepsilon A_{0} \nabla_{y}\left(E_{2} v_{2}\right)+\varepsilon A_{0} \nabla_{y}(p f)-\varepsilon A_{0} \nabla_{y}\left(q v_{t t}^{\prime \prime}\right)\right)
\end{aligned}
$$

при $y=x / \varepsilon$ и $x \in S_{2}^{\varepsilon}$. Поэтому выберем $U_{2}=N_{2 a}^{1}(y) \nabla_{x} v(x)+N_{2 b}(y) g(x)$ (см. (3.8), $(3.9))$ и определим $N_{2 a}^{1}, N_{2 b}$ как 1-периодические решения задач (3.9), в которых $E_{2}$ заменено на $E$ (равное $E_{2}^{1}$ в $Y_{2}$ ). Кроме того, продолжим $N_{2 a}^{1}, N_{2 b}$ в соответствии с (3.11). Аналогично имеем $U_{1}=N_{1 a} \nabla_{x} v+N_{1 b} g$, как и в п. 1.

Определим $\widehat{A}_{2}^{1}=\left\langle E_{2}^{T}\left(A_{2}+A_{2} \nabla_{y} N_{2 a}^{1}\right)\right\rangle_{2}$ (равное нулю в силу (3.15)). Тогда, учитывая (3.42) в (3.12), получим вместо (3.13) равенство

$$
\begin{aligned}
& R_{2} f-M_{2} v_{t t}^{\prime \prime}+\operatorname{div}_{x}\left(\widehat{A}_{2}^{1} \nabla_{x} v+\widetilde{B}_{2} g\right) \\
& \quad=-\int_{S_{2}} E_{2}^{T} \nu\left(A_{0} \nabla_{y} E_{2} v_{2}\right) d s-\int_{S_{2}} E_{2}^{T} \nu\left(A_{0} \nabla_{y}(p f)-A_{0} \nabla_{y}\left(q v_{t t}^{\prime \prime}\right)\right) d s .
\end{aligned}
$$


Пусть $\widehat{m}_{l}^{0}=\left\langle m_{0} E_{l}^{T} E\right\rangle_{\mathbf{o}}, \widehat{r}_{l}^{0}=\left\langle r_{0} E_{l}^{T} E\right\rangle_{\mathbf{o}}\left(\right.$ ср. с (3.29), (3.30)) и $\widehat{a}_{s}^{l}=\left\langle\nabla_{y} E_{l}^{T} A_{0} \nabla_{y} E_{s}\right\rangle_{\mathbf{o}}$ для $s, l=1,2$ (ср. с (3.21)). Тогда, используя $(3.21),(3.29)$ и (3.30), равенство (3.43) можно переписать в виде

$$
M_{2} v_{t t}^{\prime \prime}+\widehat{m}_{2}^{0} v_{t t}^{\prime \prime}+\widehat{a}_{2}^{2} v_{2}=R_{2} f+\widehat{r}_{2}^{0} f+\operatorname{div}_{x}\left(\widetilde{B}_{2} g\right) .
$$

Аналогично получаем вместо (3.14) равенство

$$
M_{1} v_{t t}^{\prime \prime}+\widehat{m}_{1}^{0} v_{t t}^{\prime \prime}-\operatorname{div}_{x}\left(\widehat{A}_{1} \nabla_{x} v\right)+\widehat{a}_{2}^{1} v_{2}=R_{1} f+\widehat{r}_{1}^{0} f+\operatorname{div}_{x}\left(\widehat{B}_{1} g\right) .
$$

В соответствии с (3.32) и (3.34) имеем $\widehat{a}_{2}^{1}=(-a, b)$, и

$$
\widehat{a}_{2}^{2}=\left(\begin{array}{cc}
a & -b \\
-b^{T} & d
\end{array}\right)
$$

является положительно определенной и обратимой матрицей. Учитывая (3.16) и (3.46), сложим первые $n$ уравнений из (3.44) с уравнениями из (3.45). Тогда, вспоминая о начальных и краевых условиях, получим для $v$ в точности задачу (1.15). Как уже отмечалось, достаточно гладкое решение этой задачи определено однозначно. Следовательно, можно определить достаточно гладкое $v_{2}$ как решение линейной системы уравнений $\widehat{a}_{2}^{2} v_{2}=W$ из (3.44).

Таким образом, из (3.40) имеем представление для начальных членов асимптотического разложения решения задачи (1.4):

$$
\begin{aligned}
u_{a}=v+\frac{\varepsilon^{2}}{\sigma} E_{2 \varepsilon} v_{2}+\frac{\varepsilon^{2}}{\sigma} p_{\varepsilon} f-\frac{\varepsilon^{2}}{\sigma} q_{\varepsilon} v_{t t}^{\prime \prime} & \\
& +\varepsilon N_{1 a \varepsilon} \nabla_{x} v+\varepsilon N_{1 b \varepsilon} g+\varepsilon N_{2 a \varepsilon}^{1} \nabla_{x} v+\varepsilon N_{2 b \varepsilon} g .
\end{aligned}
$$

4. Пусть выполнены условия (1.1) и (1.23). Определим $p^{0}, q^{0}, q_{1}^{0} \in H_{P}^{1}(Y)^{n \times n}$ и $q_{2}^{0} \in H_{P}^{1}(Y)^{n \times \widetilde{n}}$ как 1-периодические решения задач

$$
\begin{gathered}
-\operatorname{div}_{y}\left(A_{0} \nabla_{y} p^{0}\right)=r E \text { в } Y_{0}, \quad p^{0}=0 \text { на } \bar{Y}_{12}, \\
-\operatorname{div}_{y}\left(A_{0} \nabla_{y} q^{0}\right)=m E \text { в } Y_{0}, \quad q^{0}=0 \text { на } \bar{Y}_{12}, \\
-\operatorname{div}_{y}\left(A_{0} \nabla_{y} q_{s}^{0}\right)=m E_{s} \text { в } Y_{0}, \quad q_{s}^{0}=0 \text { на } \bar{Y}_{12}, \quad s=1,2 .
\end{gathered}
$$

Повторяя проведенные в п. 3 построения, заметим, что матричнозначные функции $p_{\varepsilon}, q_{1 \varepsilon}, q_{2 \varepsilon}$ в представлении (3.27) следует заменить на $\mu p_{\varepsilon}^{0}, \mu q_{1 \varepsilon}^{0}, \mu q_{2 \varepsilon}^{0}$ в соответствии с (1.23), (2.15) и (3.48). Такая замена отразится на вычислении коэффициентов $\widehat{m}_{s}^{l}=\mu\left\langle m E_{l}^{T} E_{s}\right\rangle_{\mathbf{0}}, \widehat{m}_{l}^{0}=\mu\left\langle m E_{l}^{T} E\right\rangle_{\mathbf{o}}$ и $\widehat{r}_{l}^{0}=\mu\left\langle r E_{l}^{T} E\right\rangle_{\mathbf{0}}$ для $s, l=1,2$, но не повлияет на дальнейшие рассуждения из п. 3.

Таким образом, представление (3.47) для начальных членов асимптотики в рассматриваемом случае можно переписать в виде

$$
\begin{aligned}
u_{a}=v+\frac{\varepsilon^{2}}{\sigma} E_{2 \varepsilon} v_{2}+\frac{\varepsilon^{2} \mu}{\sigma} & p_{\varepsilon}^{0} f-\frac{\varepsilon^{2} \mu}{\sigma} q_{\varepsilon}^{0} v_{t t}^{\prime \prime} \\
& +\varepsilon N_{1 a \varepsilon} \nabla_{x} v+\varepsilon N_{1 b \varepsilon} g+\varepsilon N_{2 a \varepsilon}^{1} \nabla_{x} v+\varepsilon N_{2 b \varepsilon} g,
\end{aligned}
$$

где $v$ является решением задачи (1.15). Коэффициенты $M$ и $R$ в этой задаче зависят от $\mu$, поскольку $\left\langle m_{0}\right\rangle_{\mathbf{o}}=\mu\langle m\rangle_{\mathbf{o}}$ и $\left\langle r_{0}\right\rangle_{\mathbf{o}}=\mu\langle r\rangle_{\mathbf{0}}$; однако такая зависимость не является сингулярной. 
Действительно, будем искать решение этой задачи в виде асимптотического ряда $v_{a}^{N}=v_{0}+\cdots+\mu^{N} v_{N}$. Тогда для $v_{0}$ получим задачу $(1.15)$, в которой $M$ и $R$ заменены на $\widehat{M}$ и $\widehat{R}$, определенные равенствами (1.24). Для $v_{s}, s=1, \ldots, N$, получаем аналогичную задачу с правой частью $\langle r\rangle_{\mathrm{o}} f \delta_{1 s}-\langle m\rangle_{\mathbf{o}}\left(v_{s-1}\right)_{t t}^{\prime \prime}$ и нулевыми начальными и граничными условиями. Поскольку исходные данные в задаче (1.15) являются гладкими и согласованными, несложно доказать, что

$$
\left\|v-v_{N}\right\|_{C^{0}\left([0, T] ; H_{0}^{1}(\Omega)^{n}\right)} \leqslant C \mu^{N+1}
$$

где постоянная $C$ не зависит от $\mu$. Аналогичная оценка выполнена для подходящего приближения $v_{2}^{N}$ к решению системы линейных уравнений из (3.44).

5. Пусть выполнены условия (1.2) и (1.23). Теперь можно повторить только начало проведенных в п. 3 построений, в соответствии с которыми матричнозначные функции $\left(\varepsilon^{2} / \sigma\right) p_{\varepsilon},\left(\varepsilon^{2} / \sigma\right) q_{1 \varepsilon},\left(\varepsilon^{2} / \sigma\right) q_{2 \varepsilon}$ в разложении $(3.27)$ следует заменить на $(\mu / \vartheta) p_{\varepsilon}^{0},(\mu / \vartheta) q_{1 \varepsilon}^{0}$ и $(\mu / \vartheta) q_{2 \varepsilon}^{0}$. Тогда для $v_{1}, v_{2}$ получаем начально-краевую задачу (3.31) с положительной постоянной $\vartheta$ из условия (1.2) в равенствах (3.21). Коэффициенты этой задачи зависят от $\mu$, но не сингулярным образом, поскольку $\widehat{m}_{s}^{l}=\mu\left\langle m E_{l}^{T} E_{s}\right\rangle_{\mathbf{o}}$ и $\widehat{r}_{l}^{0}=\mu\left\langle r E_{l}^{T} E\right\rangle_{\mathbf{o}}$ для $s, l=1,2$. В первом приближении можно выбрать $\widehat{m}_{s}^{l}=0, \widehat{r}_{l}^{0}=0$ и определить начальные члены асимптотики равенством

$$
u_{a}=E_{1 \varepsilon} v_{1}+E_{2 \varepsilon} v_{2}+\frac{\mu}{\vartheta} p_{\varepsilon}^{0} f-\frac{\mu}{\vartheta} q_{1 \varepsilon}^{0} v_{1}^{\prime \prime}-\frac{\mu}{\vartheta} q_{2 \varepsilon}^{0} v_{2}^{\prime \prime}+\varepsilon u_{1}
$$

где $v_{1}, v_{2}$ являются решениями задачи $(1.21)$, в которой $M_{s}^{l}=0$ и $R_{l}^{0}=0$ для $s, l=1,2$. Отметим, что выбор в (3.51) решения $v_{1}, v_{2}$ "более точной" задачи (3.31) не улучшает оценки теоремы 5 , поскольку при переходе от разложения (3.27) (фактически (3.22)) к разложению (3.51) использованы леммы 5 и 7 , гарантирующие только точность $C(\mu / \vartheta)^{2}$.

6. Пусть вьполнены условия (1.3), (1.23) и (1.25). Поскольку из лемм 5 и 7 еще следуют асимптотические равенства, аналогичные (3.26), можно повторить начало построений из п. 3. Тогда матричнозначные функции $p_{\varepsilon}, q_{1 \varepsilon}, q_{2 \varepsilon}$ в разложении (3.27) следует заменить на $\mu p_{\varepsilon}^{0}, \mu q_{1 \varepsilon}^{0}, \mu q_{2 \varepsilon}^{0}$ из (3.48) и определить $v_{1}, v_{2}$ как решения задачи (3.31). В соответствии с определениями (3.21), (3.29) и (3.30) коэффициенты этой задачи зависят от $\mu$ и $\sigma / \varepsilon^{2}$, но не сингулярным образом. В первом приближении можно выбрать $\widehat{m}_{s}^{l}=0, \widehat{r}_{l}^{0}=0, a_{s}^{l}=0$ для $s, l=1,2$ и определить начальные члены асимптотики равенством

$$
u_{a}=E_{1 \varepsilon} v_{1}+E_{2 \varepsilon} v_{2}+\frac{\varepsilon^{2} \mu}{\sigma} p_{\varepsilon}^{0} f-\frac{\varepsilon^{2} \mu}{\sigma} q_{1 \varepsilon}^{0} v_{1}^{\prime \prime}-\frac{\varepsilon^{2} \mu}{\sigma} q_{2 \varepsilon}^{0} v_{2}^{\prime \prime}+\varepsilon u_{1}
$$

где $v_{1}, v_{2}$ являются решениями задач (1.22).

7. Пусть выполнены условия (1.3), (1.23) и (1.26). Вернемся к построениям п. 1 этого параграфа. Тогда равенство (3.17) для определения $V_{0}$ в рассматриваемом случае принимает вид

$$
\begin{aligned}
\rho^{2} m V_{0}-\theta \operatorname{div}_{y}( & \left.A_{0} \nabla_{y} V_{0}\right)=r F+m E_{1}\left(\rho w_{0}+w_{1}-\rho^{2} V_{1}\right) \\
& +m E_{2}\left(\rho \widetilde{w}_{0}+\widetilde{w}_{1}-\rho^{2} V_{2}\right) \text { при } y=x / \varepsilon \quad \text { и } x \in \Omega_{0}^{\varepsilon},
\end{aligned}
$$


поскольку $\theta-\sigma /\left(\varepsilon^{2} \mu\right)$ является малым параметром. Определим $P^{0}, Q_{1}^{0}$ и $Q_{2}^{0}$ как 1-периодические решения задач $(1.19)$, в которых $m_{0}, r_{0}$ заменены на $m, r$ и $\vartheta$ заменена на $\theta$. Из (3.53), как и в п. 1 , получаем представление

$$
u_{a}=E_{1 \varepsilon} v_{1}+E_{2 \varepsilon} v_{2}+P_{\varepsilon}^{0} * f-Q_{1 \varepsilon}^{0} *\left(v_{1}^{\prime \prime}\right)-Q_{2 \varepsilon}^{0} *\left(v_{2}^{\prime \prime}\right)+\varepsilon u_{1},
$$

где $v_{1}$ и $v_{2}$ определяются из соотношений (3.13) и (3.14). В этих соотношениях $\vartheta=\sigma / \varepsilon^{2}$ является малым параметром в силу условия (1.3) (см. п. 2.). Поэтому в (3.13) и (3.14) граничные интегралы являются несушественными и для определения $v_{1}, v_{2}$ получаем начально-краевые задачи (1.22).

8. Пусть выполнены условия (1.3), (1.23) и (1.27). Теперь можно воспользоваться леммами 6,7 и построениями из п. 7. В силу этих лемм вьполнены равенства, аналогичные (3.24). Следовательно, используя эти равенства в (3.54), получаем для начальных членов асимптотики представление (3.25), в котором $v_{1}, v_{2}$ являются решениями задач $(1.22)$ и $r_{0} / m_{0}$ заменено на $r / m$.

ЗАмЕчАНИЕ 5 . Пусть имеются $s_{1}$ связных множеств $F_{1}, \ldots, F_{s_{1}}, s_{2}$ вполне несвязных множеств $F_{s_{1}+1}, \ldots, F_{s_{1}+s_{2}}$, определенных в замечании 3 . Кроме того, пусть $F_{0}=\mathbb{R}^{n} \backslash\left(\bar{F}_{1} \cup \cdots \cup \bar{F}_{s_{1}+s_{2}}\right)$. Для $l=0, \ldots, s_{1}+s_{2}$ множества $\Omega_{l}^{\varepsilon}$ и $S_{l}^{\varepsilon}$ определяются так же, как и в (1.6), (3.1). Естественно предполагается, что малый параметр $\sigma$ входит только в определения (1.6) на $\Omega_{0}^{\varepsilon}$. Поэтому $s=1, \ldots, s_{1}+s_{2}$ в задаче (3.3). Для таких $s$ определим $E_{s}(y)$ как 1-периодическое решение задач

$$
\operatorname{div}_{y}\left(A_{0} \nabla_{y} E_{s}\right)=0 \text { в } Y_{0}, \quad E_{s}=\delta_{s l} E_{0}^{s} \text { на } \bar{Y}_{l}, \quad l=1, \ldots, s_{1}+s_{2},
$$

где $E_{0}^{s}=E$ для $s=1, \ldots, s_{1}$, а для $s=s_{1}+1, \ldots, s_{1}+s_{2}$ столбцы матрицы $E_{0}^{s}$ образуют базис в линейном пространстве жестких перемешений $\Re$, нормированный условиями, аналогичными (1.17). Для построения начальных членов асимптотики решения задачи (1.4) в рассматриваемом случае необходимо фактически повторить построения этого параграфа, в которых 1 и 2 заменены на $l_{1}=1, \ldots, s_{1}$ и $l_{2}=s_{1}+1, \ldots, s_{1}+s_{2}$ соответственно. Тогда осредненные задачи имеют вид, описанный в замечании 3 и зависящий от выполнения одного из условий (1.1)-(1.3). Аналогично рассматривается и случай, когда выполнено условие (1.23).

\section{§4. Доказательство теоремы 2}

В случае, когда выполнено условие (1.2), начальные члены асимптотического разложения решения задачи (1.4) определим равенством (3.22). В этом равенстве все слагаемые, кроме $\varepsilon N_{1 a \varepsilon} \nabla v_{1}$ и $\varepsilon N_{2 a \varepsilon} \nabla v_{2}$, удовлетворяют однородным граничным условиям Дирихле на $\partial \Omega$. Полезно подправить и эти слагаемые в (3.22). Для этого определим гладкую в $\Omega$ функцию $\eta$, равную нулю при $\operatorname{dist}(x, \partial \Omega) \leqslant \varepsilon$ и единице при $\operatorname{dist}(x, \partial \Omega) \geqslant 2 \varepsilon$, где $\operatorname{dist}(x, \partial \Omega)$ обозначает расстояние от точки $x$ до границы $\partial \Omega$. Для достаточно малых $\varepsilon\left(\leqslant \varepsilon_{0}\right)$ такие функции сушествуют, $\varepsilon|\nabla \eta| \leqslant C$ в $\Omega$, и носители функций $\eta-1$ и $\nabla \eta$ содержатся в приграничной полосе $\Omega_{2 \varepsilon} \subset \Omega$ ширины $2 \varepsilon$ (см., например, [4]). Тогда подправленная асимптотика задается равенством

$$
\begin{aligned}
v_{a}=E_{1 \varepsilon} v_{1}+E_{2 \varepsilon} v_{2}+P_{\varepsilon} * f-Q_{1 \varepsilon} *\left(\left(v_{1}\right)_{t t}^{\prime \prime}\right)-Q_{2 \varepsilon} *\left(\left(v_{2}\right)_{t t}^{\prime \prime}\right) \\
+\varepsilon\left(N_{1 a}\right)_{\varepsilon} \eta \nabla v_{1}+\varepsilon\left(N_{1 b}\right)_{\varepsilon} g+\varepsilon\left(N_{2 a}\right)_{\varepsilon} \eta \nabla v_{2}+\varepsilon\left(N_{2 b}\right)_{\varepsilon} g
\end{aligned}
$$


где $\left(v_{1}, v_{2}\right)$ является решением задачи $(1.21)$ и, например, $\left(N_{1 a}\right)_{\varepsilon}=N_{1 a}(x / \varepsilon)$ обозначает $\varepsilon$-периодическое продолжение этой функции на $\Omega$ (см. $\S 2)$.

Из ограниченности области $\Omega$ и известной оценки $\operatorname{mes}\left(\Omega_{2 \varepsilon}\right) \leqslant C \varepsilon$ следуют неравенства

$$
\begin{gathered}
\left\|\left(N_{1 a}\right)_{\varepsilon}\right\|_{L^{2}(\Omega)^{n \times n}}^{2}+\left\|\left(\nabla_{y} N_{1 a}\right)_{\varepsilon}\right\|_{L^{2}(\Omega)^{n \times n \times n}}^{2} \leqslant C, \\
\left\|\left(N_{1 a}\right)_{\varepsilon}\right\|_{L^{2}\left(\Omega_{2 \varepsilon}\right)^{n \times n}}^{2}+\left\|\left(\nabla_{y} N_{1 a}\right)_{\varepsilon}\right\|_{L^{2}\left(\Omega_{2 \varepsilon}\right)^{n \times n \times n}}^{2} \leqslant C \varepsilon
\end{gathered}
$$

и аналогичные неравенства для матричнозначных функций $E_{1}, E_{2}, N_{1 b}, N_{2 a}$ и $N_{2 b}$ (см., например, [11]). Напомним, что эти функции определяются в (1.11), (1.13), $(1.16),(1.18),(3.9),(3.11)$ и имеют компоненты из $H_{P}^{1}(Y)$.

Из ограниченности области $\Omega$ и леммы 4 следует также неравенство

$$
\left\|\left(P_{t}^{\prime}\right)_{\varepsilon}\right\|_{C^{0}\left([0, T] ; L^{2}(\Omega)^{n \times n}\right)}^{2}+\vartheta\left\|\left(\nabla_{y} P\right)_{\varepsilon}\right\|_{C^{0}\left([0, T] ; L^{2}(\Omega)^{n \times n \times n}\right)}^{2} \leqslant C
$$

и аналогичные неравенства для $Q_{1}$ и $Q_{2}$. В этих неравенствах и далее в этом параграфе постоянная $C$ не зависит от $\varepsilon, \sigma$ и $\vartheta$. Из равенств (2.20) имеем, например, $Q_{1 \varepsilon} *\left(v_{1}^{\prime \prime}\right)=\left(Q_{1}^{\prime}\right)_{\varepsilon} *\left(v_{1}^{\prime}\right)$. Поэтому слагаемые со свертками в (4.1) определены как элементы пространства $C^{1}\left([0, T] ; H_{0}^{1}(\Omega)^{n}\right)$ в силу леммы 7 , неравенств $(4.3)$ и гладкости $v_{1}, v_{2}$ и $f$.

Следуюший вариант первой формулы из (3.6) и различные его вариации в обозначениях часто используется в дальнейшем:

$$
\nabla\left(P_{\varepsilon} * f\right)=\varepsilon^{-1}\left(\nabla_{y} P\right)_{\varepsilon} * f+P_{\varepsilon} *(\nabla f)
$$

Пусть $u$ является решением задачи (1.4). Введем обозначения

$$
r=u-v_{a}, \quad r_{a}=v_{a}-u_{a} .
$$

Учитывая $(3.21),(4.1)$ и $(4.4)$, имеем $r_{a}=\varepsilon\left(N_{s a}\right)_{\varepsilon}(\eta-1) \nabla v_{s}$ и

$$
\nabla\left(r_{a}\right)=\left(\nabla_{y} N_{s a}\right)_{\varepsilon}(\eta-1) \nabla v_{s}+\varepsilon\left(N_{s a}\right)_{\varepsilon}(\nabla \eta) \nabla v_{s}+\varepsilon\left(N_{s a}\right)_{\varepsilon}(\eta-1) \nabla^{2} v_{s} .
$$

Здесь и далее предполагается суммирование по повторяющимся индексам $s=1,2$. Следовательно, используя второе неравенство из (4.2) и гладкость $v_{1}, v_{2}$, получаем

$$
\begin{aligned}
& \left\|\nabla\left(r_{a}\right)\right\|_{C^{0}\left([0, T] ; L^{2}(\Omega)^{n \times n}\right)}^{2}+\left\|\nabla\left(r_{a}\right)_{t}^{\prime}\right\|_{L^{1}\left(0, T ; L^{2}(\Omega)^{n \times n}\right)}^{2} \\
& \quad+\left\|r_{a}\right\|_{C^{0}\left([0, T] ; L^{2}(\Omega)^{n}\right)}^{2}+\left\|\left(r_{a}\right)_{t}^{\prime}\right\|_{L^{1}\left(0, T ; L^{2}(\Omega)^{n}\right)}^{2} \leqslant C \varepsilon,
\end{aligned}
$$

так что $v_{a}$ достаточно близка к асимптотике $u_{a}$ и $v_{a}=0$ на $\partial \Omega \times(0, T)$.

Выберем $\varphi=r_{t}^{\prime}$ в интегральном тождестве (2.4) и вычтем соответствуюшие интегралы от $v_{a}$. Тогда

$$
\begin{aligned}
& \left(\frac{1}{2} \int_{\Omega} m_{\varepsilon}\left|r_{t}^{\prime}\right|^{2} d x\right)_{t}^{\prime}+\left(\frac{1}{2} \int_{\Omega}\left(A_{\sigma}^{\varepsilon} e(r), e(r)\right) d x\right)_{t}^{\prime} \\
& =\int_{\Omega}\left(r_{\varepsilon} f, r_{t}^{\prime}\right) d x-\int_{\Omega}\left(m_{\varepsilon}\left(v_{a}\right)_{t t}^{\prime \prime}, r_{t}^{\prime}\right) d x-\int_{\Omega}\left(A_{\sigma}^{\varepsilon} e\left(v_{a}\right)+B_{\sigma}^{\varepsilon} g, e\left(r_{t}^{\prime}\right)\right) d x \equiv I_{t} .
\end{aligned}
$$


В силу условий теоремы и определений (4.1), (4.5) имеем при $t=0$ равенства $r=0$ и $r_{t}^{\prime}=0$. Поэтому так же, как и в доказательстве леммы 1 , получаем

$$
\left\|r_{t}^{\prime}\right\|_{L^{2}(\Omega)^{n}}^{2}+\sigma\|e(r)\|_{L^{2}\left(\Omega_{0}^{\varepsilon}\right)^{n \times n}}^{2}+\|e(r)\|_{L^{2}\left(\Omega_{12}^{\varepsilon}\right)^{n \times n}}^{2} \leqslant \frac{2}{\alpha}\left|\int_{0}^{t} I_{t} d t\right| \equiv I .
$$

Воспользуемся соотношениями (2.9) для $r$. Тогда для доказательства теоремы 2 достаточно получить неравенство

$$
I \leqslant \frac{1}{2} \mathscr{E}(u)+C\left(\varepsilon(1+\vartheta+\sqrt{\vartheta})+\sigma+\varepsilon \frac{1+\sqrt{\vartheta}}{\sqrt{\sigma \vartheta}}\left|\vartheta-\frac{\sigma}{\varepsilon^{2}}\right|\right),
$$

где постоянная $C$ не зависит от $\varepsilon, \sigma$ и $\vartheta$ (см. доказательство леммы 1$)$.

Введем обозначение $g_{s}=g$. Тогда, используя (4.4) и (4.5), имеем

$$
\begin{aligned}
\nabla v_{a}= & \nabla r_{a}+\nabla u_{a}=\nabla r_{a}+\varepsilon^{-1}\left(\nabla_{y} E_{s}\right)_{\varepsilon} v_{s}+\varepsilon^{-1}\left(\nabla_{y} P\right)_{\varepsilon} * f \\
& -\varepsilon^{-1}\left(\nabla_{y} Q_{s}\right)_{\varepsilon} *\left(v_{s}^{\prime \prime}\right)+\left(\nabla_{y} N_{s a}\right)_{\varepsilon} \nabla v_{s}+\left(\nabla_{y} N_{s b}\right)_{\varepsilon} g_{s}+\left(E_{s}\right)_{\varepsilon} \nabla v_{s} \\
& +P_{\varepsilon} *(\nabla f)-Q_{s \varepsilon} *\left(\nabla\left(v_{s}^{\prime \prime}\right)\right)+\varepsilon\left(N_{s a}\right)_{\varepsilon} \nabla^{2} v_{s}+\varepsilon\left(N_{s b}\right)_{\varepsilon} \nabla g_{s} \\
\equiv & \nabla r_{a}+\varepsilon^{-1}\left(\nabla_{y} E_{s}\right)_{\varepsilon} v_{s}+\varepsilon^{-1}\left(\nabla_{y} P\right)_{\varepsilon} * f-\varepsilon^{-1}\left(\nabla_{y} Q_{s}\right)_{\varepsilon} *\left(v_{s}^{\prime \prime}\right) \\
& +\left(E_{s}+\nabla_{y} N_{s a}\right)_{\varepsilon} \nabla v_{s}+\left(\nabla_{y} N_{s b}\right)_{\varepsilon} g_{s}+P_{\varepsilon} *(\nabla f)-Q_{s \varepsilon} *\left(\nabla\left(v_{s}^{\prime \prime}\right)\right)+\varepsilon J .
\end{aligned}
$$

Здесь и далее $\|J\|_{C^{0}\left([0, T] ; L^{2}(\Omega)\right)}+\left\|J_{t}^{\prime}\right\|_{L^{1}\left(0, T ; L^{2}(\Omega)\right)} \leqslant C$ в силу первого неравенства из (4.2) и гладкости $v_{1}, v_{2}$ и $g$.

По определению $E_{1 \varepsilon}, P_{\varepsilon}, Q_{s \varepsilon}, N_{1 a \varepsilon}$ и $N_{1 b \varepsilon}$ имеют нулевые компоненты на $\bar{\Omega}_{2}^{\varepsilon}$. Кроме того, вьполнено равенство $\left(A_{2} \nabla_{y} E_{2} v_{2}\right)_{\varepsilon}=0$ на $\Omega_{2}^{\varepsilon}$ (см. 33$)$. Следовательно,

$$
A_{\varepsilon}^{\sigma} \nabla v_{a}+B_{\varepsilon}^{\sigma} g=A_{\varepsilon}^{\sigma} \nabla r_{a}+\varepsilon A_{\varepsilon}^{\sigma} J+\left(\widetilde{A}_{2 a}\right)_{\varepsilon} \nabla v_{2}+\left(\widetilde{A}_{2 b}\right)_{\varepsilon} g \text { в } \Omega_{2}^{\varepsilon},
$$

где $\widetilde{A}_{2 a}(y)=A_{2} E_{2}+A_{2} \nabla_{y} N_{2 a}$ и $\widetilde{A}_{2 b}(y)=B_{2}+A_{2} \nabla_{y} N_{2 b}$ удовлетворяют соотношениям:

$$
\begin{gathered}
\left\langle E_{2}^{T} \widetilde{A}_{2 a}\right\rangle_{2}=\widehat{A}_{2}, \quad\left\langle E_{2}^{T} \widetilde{A}_{2 b}\right\rangle_{2}=\widetilde{B}_{2}, \\
\operatorname{div} \widetilde{A}_{2 a}=0, \operatorname{div}_{y} \widetilde{A}_{2 b}=0 \text { в } Y_{2}, \quad \nu \widetilde{A}_{2 a}=0, \nu \widetilde{A}_{2 b}=0 \text { на } S_{2}
\end{gathered}
$$

в соответствии с $(1.13),(1.20),(3.9)$ и (3.13). Аналогично получаем

$$
A_{\varepsilon}^{\sigma} \nabla v_{a}+B_{\varepsilon}^{\sigma} g=A_{\varepsilon}^{\sigma} \nabla r_{a}+\varepsilon A_{\varepsilon}^{\sigma} J+\left(\widetilde{A}_{1 a}\right)_{\varepsilon} \nabla v_{1}+\left(\widetilde{A}_{1 b}\right)_{\varepsilon} g \quad \text { в } \quad \Omega_{1}^{\varepsilon},
$$

где $\widetilde{A}_{1 a}(y)=A_{1} E_{1}+A_{1} \nabla_{y} N_{1 a}$ и $\widetilde{A}_{1 b}(y)=B_{1}+A_{1} \nabla_{y} N_{1 b}$ удовлетворяют соотношениям (4.11), в которых $\widetilde{B}_{2}$ заменен на $\widehat{B}_{1}$ и 2 заменена на 1 .

Соответствующие интегралы по $\Omega \times(0, t)$ из $(4.7)$, содержащие $\nabla r_{a}$ и $\varepsilon J$, несущественны в (4.8). Действительно, учитывая (4.6) и доказательство неравенств (2.6)-(2.8), получаем

$$
\begin{aligned}
& \left|\int_{0}^{t} \int_{\Omega}\left(A_{\varepsilon}^{\sigma} e\left(r_{a}\right), e\left(r_{t}^{\prime}\right)\right) d x d t\right| \\
& \quad \leqslant \frac{C}{2 \delta}\left\|\nabla r_{a}\right\|_{C^{0}\left([0, T] ; L^{2}(\Omega)^{n \times n}\right)}^{2}+\frac{C}{2 \delta}\left\|\nabla\left(r_{a}\right)_{t}^{\prime}\right\|_{L^{1}\left(0, T ; L^{2}(\Omega)^{n \times n}\right)}^{2}+\delta C \mathscr{E}(r) \\
& \quad \leqslant C \varepsilon+\delta_{1} \mathscr{E}(r),
\end{aligned}
$$

где $\delta=\delta_{1} / C$. Здесь и далее положительные постоянные $\delta_{1}, \ldots, \delta_{l}$ будут выбраны так, чтобы $(2 / \alpha)\left(\delta_{1}+\cdots+\delta_{l}\right)=1 / 2($ см. $(4.7)$ и $(4.8))$. 
Используя первое равенство из (2.20) и начальные условия из (1.19), имеем

$$
\begin{aligned}
\left(v_{a}\right)_{t t}^{\prime \prime}= & E_{s \varepsilon}\left(v_{s}\right)_{t t}^{\prime \prime}+\left(P_{t t}^{\prime \prime}\right)_{\varepsilon} * f+\left(\left.P_{t}^{\prime}\right|_{t=0}\right)_{\varepsilon} f \\
& -\left(\left(Q_{s}\right)_{t t}^{\prime \prime}\right)_{\varepsilon} *\left(\left(v_{s}\right)_{t t}^{\prime \prime}\right)-\left(\left.\left(Q_{s}\right)_{t}^{\prime}\right|_{t=0}\right)_{\varepsilon}\left(\left(v_{s}\right)_{t t}^{\prime \prime}\right)+\varepsilon J \\
= & \left(P_{t t}^{\prime \prime}\right)_{\varepsilon} * f+\left(r_{0} / m_{0}\right)_{\varepsilon} f-\left(\left(Q_{s}\right)_{t t}^{\prime \prime}\right)_{\varepsilon} *\left(\left(v_{s}\right)_{t t}^{\prime \prime}\right)+\varepsilon J \text { в } \Omega_{0}^{\varepsilon} \\
\left(v_{a}\right)_{t t}^{\prime \prime}= & E_{1 \varepsilon}\left(v_{1}\right)_{t t}^{\prime \prime}+\varepsilon J \text { в } \Omega_{1}^{\varepsilon}, \quad\left(v_{a}\right)_{t t}^{\prime \prime}=E_{2 \varepsilon}\left(v_{2}\right)_{t t}^{\prime \prime}+\varepsilon J \text { в } \Omega_{2}^{\varepsilon}
\end{aligned}
$$

где интегралы из (4.7), содержащие $J$, несущественны в (4.8).

Выделяя сингулярные слагаемые в (4.9), введем обозначения

$$
\begin{gathered}
u^{0}=\varepsilon^{-1}\left(\nabla_{y} P\right)_{\varepsilon} * f-\varepsilon^{-1}\left(\nabla_{y} Q_{s}\right)_{\varepsilon} *\left(\left(v_{s}\right)_{t t}^{\prime \prime}\right), \\
u^{1}=\varepsilon^{-1}\left(\nabla_{y} E_{s}\right)_{\varepsilon} v_{s}, \quad u^{2}=u^{0}+u^{1}
\end{gathered}
$$

и определим $u^{3}$ из равенства

$$
A_{\varepsilon}^{\sigma} \nabla v_{a}=A_{\varepsilon}^{\sigma} u^{2}+A_{\varepsilon}^{\sigma} u^{3} \text { в } \Omega_{0}^{\varepsilon}
$$

Компоненты тензора $A_{\varepsilon}^{\sigma}$ имеют порядок $\sigma$ на $\Omega_{0}^{\varepsilon}$, а компоненты вектор-функции $u^{3}$ имеют порядок единицы. Поэтому, учитывая $(4.2),(4.3),(4.6),(4.9)$, гладкость $v_{s}$, $f, g$ и лемму 7, имеем

$$
\begin{aligned}
& \left|\int_{0}^{t} \int_{\Omega_{0}^{\varepsilon}}\left(A_{\varepsilon}^{\sigma} u^{3}, e\left(r_{t}^{\prime}\right)\right) d x d t\right| \\
& \quad \leqslant \frac{\sigma C}{2 \delta}\left\|u^{3}\right\|_{C^{0}\left([0, T] ; L^{2}\left(\Omega_{0}^{\varepsilon}\right)^{n \times n}\right)}^{2}+\frac{\sigma C}{2 \delta}\left\|\left(u^{3}\right)_{t}^{\prime}\right\|_{L^{1}\left(0, T ; L^{2}\left(\Omega_{0}^{\varepsilon}\right)^{n \times n}\right)}^{2}+\delta C \mathscr{E}(r) \\
& \quad \leqslant C \sigma+\delta_{2} \mathscr{E}(r),
\end{aligned}
$$

где $\delta=\delta_{2} / C$. Следовательно, этот интеграл и аналогичный интеграл по $\Omega_{0}^{\varepsilon} \times(0, t)$ из (4.7), содержащий $B_{\sigma}^{\varepsilon} g$, несушественны в (4.8). При выводе неравенства (4.17) использовано, что $u^{3}$ не содержит градиентов от $P_{\varepsilon}$ и $Q_{s \varepsilon}$, оценки которых могут зависеть от $\vartheta$.

Таким образом, учитывая (4.10)-(4.17) в неравенстве (4.7), получаем

$$
\begin{aligned}
I \leqslant & \int_{0}^{t}\left[\int_{\Omega_{0}^{\varepsilon}}\left(\left(m_{0} Q_{s}^{\prime \prime}\right)_{\varepsilon} *\left(v_{s}^{\prime \prime}\right), r_{t}^{\prime}\right) d x-\int_{\Omega_{0}^{\varepsilon}}\left(\left(m_{0} P^{\prime \prime}\right)_{\varepsilon} * f, r_{t}^{\prime}\right) d x\right. \\
& -\int_{\Omega_{0}^{\varepsilon}}\left(A_{\varepsilon}^{\sigma} u^{2}, e\left(r_{t}^{\prime}\right)\right) d x-\int_{\Omega_{2}^{\varepsilon}}\left(\left(\widetilde{A}_{2 a}\right)_{\varepsilon} \nabla v_{2}+\left(\widetilde{A}_{2 b}\right)_{\varepsilon} g, e\left(r_{t}^{\prime}\right)\right) d x \\
& +\int_{\Omega_{2}^{\varepsilon}}\left(\left(r_{2}\right)_{\varepsilon} f, r_{t}^{\prime}\right) d x-\int_{\Omega_{2}^{\varepsilon}}\left(\left(m_{2} E_{2}\right)_{\varepsilon} v_{2}^{\prime \prime}, r_{t}^{\prime}\right) d x+\int_{\Omega_{1}^{\varepsilon}}\left(\left(r_{1}\right)_{\varepsilon} f, r_{t}^{\prime}\right) d x \\
& \left.-\int_{\Omega_{1}^{\varepsilon}}\left(\left(m_{1} E_{1}\right)_{\varepsilon} v_{1}^{\prime \prime}, r_{t}^{\prime}\right) d x-\int_{\Omega_{1}^{\varepsilon}}\left(\left(\widetilde{A}_{1 a}\right)_{\varepsilon} \nabla v_{1}+\left(\widetilde{A}_{1 b}\right)_{\varepsilon} g, e\left(r_{t}^{\prime}\right)\right) d x\right] d t \\
& +C(\varepsilon+\sigma)+\left(\delta_{1}+\delta_{2}\right) \mathscr{E}(r) .
\end{aligned}
$$


В силу определений $r$ удовлетворяет однородным граничным условиям Дирихле на $\partial \Omega$, и граница $S_{2}^{\varepsilon}=\partial \Omega_{2}^{\varepsilon} \backslash \partial \Omega$ имеет класс $C^{2}$. Поэтому в области $\Omega_{2}^{\varepsilon}$ выполнена следуюшая формула интегрирования по частям:

$$
\int_{\Omega_{2}^{\varepsilon}}\left(\left(\widetilde{A}_{2 b}\right)_{\varepsilon} g, e\left(r_{t}^{\prime}\right)\right) d x=\int_{S_{2}^{\varepsilon}}\left(\left(\nu \widetilde{A}_{2 b}\right)_{\varepsilon} g, r_{t}^{\prime}\right) d s-\int_{\Omega_{2}^{\varepsilon}}\left(\operatorname{div}\left(\left(\widetilde{A}_{2 b}\right)_{\varepsilon} g\right), r_{t}^{\prime}\right) d x
$$

(см. формулы $(2.10),(2.12)$ и [3]). Отметим, что $\left(\nu \widetilde{A}_{2 b}\right)_{\varepsilon}=0$ на $S_{2}^{\varepsilon}$ в соответствии с (4.11). Из (3.6) следует, что

$$
\operatorname{div}\left(\left(\widetilde{A}_{2 b}\right)_{\varepsilon} g\right)=\varepsilon^{-1}\left(\operatorname{div}_{y} \widetilde{A}_{2 b}\right)_{\varepsilon} g+\left(\widetilde{A}_{2 b}\right)_{\varepsilon} \nabla g .
$$

Таким образом, используя (4.11), (4.19) и (4.20), заключаем, что

$$
\begin{aligned}
I_{2} & \equiv \int_{\Omega_{2}^{\varepsilon}}\left(\left(r_{2}\right)_{\varepsilon} f-\left(m_{2} E_{2}\right)_{\varepsilon} v_{2}^{\prime \prime}, r_{t}^{\prime}\right) d x-\int_{\Omega_{2}^{\varepsilon}}\left(\left(\widetilde{A}_{2 a}\right)_{\varepsilon} \nabla v_{2}+\left(\widetilde{A}_{2 b}\right)_{\varepsilon} g, e\left(r_{t}^{\prime}\right)\right) d x \\
& =\int_{\Omega_{2}^{\varepsilon}}\left(\left(r_{2}\right)_{\varepsilon} f-\left(m_{2} E_{2}\right)_{\varepsilon} v_{2}^{\prime \prime}, r_{t}^{\prime}\right) d x-\int_{\Omega_{2}^{\varepsilon}}\left(\left(\widetilde{A}_{2 a}\right)_{\varepsilon} \nabla^{2} v_{2}+\left(\widetilde{A}_{2 b}\right)_{\varepsilon} \nabla g, r_{t}^{\prime}\right) d x .
\end{aligned}
$$

Определим $E_{2}^{0}(y)=\left(m_{2}(y) E_{2}(y)\right) / M_{2}$. Эта матричнозначная функция примечательна тем, что $\left\langle E_{2}^{T} E_{2}^{0}\right\rangle_{2}=\widetilde{E}$ в соответствии с $(1.17)$ и (1.20). Введем также обозначения для матричнозначных функций

$$
\begin{gathered}
r_{2}^{0}(y)=r_{2}(y) E-E_{2}^{0}(y) R_{2}, \quad m_{2}^{0}(y)=m_{2}(y) E_{2}(y)-E_{2}^{0}(y) M_{2}, \\
A_{2 a}^{0}(y)=\widetilde{A}_{2 a}(y)-E_{2}^{0}(y) \widehat{A}_{2}, \quad A_{2 b}^{0}(y)=\widetilde{A}_{2 b}(y)-E_{2}^{0}(y) \widetilde{B}_{2},
\end{gathered}
$$

ортогональных на $Y_{2}$ матричнозначной функции $E_{2}(y)$ :

$$
\left\langle E_{2}^{T} r_{2}^{0}\right\rangle_{2}=0, \quad\left\langle E_{2}^{T} m_{2}^{0}\right\rangle_{2}=0, \quad\left\langle E_{2}^{T} A_{2 a}^{0}\right\rangle_{2}=0, \quad\left\langle E_{2}^{T} A_{2 b}^{0}\right\rangle_{2}=0 .
$$

Напомнив, что $\widehat{A}_{2}=0$ в силу $(3.15)$, перепишем второе уравнение из $(1.21)$ в виде

$$
M_{2} v_{2}^{\prime \prime}-M_{s}^{2} *\left(v_{s}^{\prime \prime}\right)-\widehat{A}_{2} \nabla^{2} v_{2}+a_{s}^{2} v_{s}-R_{2} f+R_{2}^{0} * f-\widetilde{B}_{2} \nabla g=0 .
$$

Умножим (4.24) слева на $E_{2 \varepsilon}^{0}$ и справа на $r_{t}^{\prime}$, проинтегрируем полученное соотношение по $\Omega_{2}^{\varepsilon}$ и учтем результат в (4.21). Тогда, используя (4.22), равенство (4.21) можно переписать в виде

$$
\begin{aligned}
I_{2}= & \int_{\Omega_{2}^{\varepsilon}}\left(\left(E_{2}^{0} R_{2}^{0}\right)_{\varepsilon} * f+\left(E_{2}^{0} a_{s}^{2}\right)_{\varepsilon} v_{s}-\left(E_{2}^{0} M_{s}^{2}\right)_{\varepsilon} *\left(v_{s}^{\prime \prime}\right), r_{t}^{\prime}\right) d x \\
& +\int_{\Omega_{2}^{\varepsilon}}\left(\left(r_{2}^{0}\right)_{\varepsilon} f, r_{t}^{\prime}\right) d x-\int_{\Omega_{2}^{\varepsilon}}\left(\left(A_{2 a}^{0}\right)_{\varepsilon} \nabla^{2} v_{2}+\left(A_{2 b}^{0}\right)_{\varepsilon} \nabla g+\left(m_{2}^{0}\right)_{\varepsilon} v_{2}^{\prime \prime}, r_{t}^{\prime}\right) d x .
\end{aligned}
$$

Последние два интеграла из (4.25) несушественны в (4.18). Действительно, рассмотрим, например, первьй из этих интегралов. Определим $U \in\left(H_{P}^{1}\left(Y_{2}\right)^{n} \cap\right.$ $\left.L^{2}\left(Y_{2}\right)^{n} / \Re\right)^{n}$ как 1-периодическое решение задачи Неймана на $Y_{2}$ :

$$
-\operatorname{div}_{y}\left(A_{2} \nabla_{y} U\right)=r_{2}^{0} \text { в } Y_{2}, \quad-\nu\left(A_{2} \nabla_{y} U\right)=0 \text { на } S_{2} .
$$


В силу (4.23) и леммы 3 такое решение существует и удовлетворяет оценке

$$
\left\|\left(A_{2} \nabla_{y} U\right)_{\varepsilon}\right\|_{L^{2}\left(\Omega_{2}^{\varepsilon}\right)^{n \times n \times n}} \leqslant C
$$

поскольку область $\Omega_{2}^{\varepsilon} \subset \Omega$ ограничена и для $A_{2}$ выполнено условие эллиптичности (1.7). Следовательно, используя (4.19), (4.20) и (4.26), имеем

$$
\begin{aligned}
\int_{\Omega_{2}^{\varepsilon}}\left(\left(r_{2}^{0}\right)_{\varepsilon} f, r_{t}^{\prime}\right) d x & =-\int_{\Omega_{2}^{\varepsilon}}\left(\left(\operatorname{div}_{y}\left(A_{2} \nabla_{y} U\right)\right)_{\varepsilon} f, r_{t}^{\prime}\right) d x \\
& =\varepsilon \int_{\Omega_{2}^{\varepsilon}}\left(\left(A_{2} \nabla_{y} U\right)_{\varepsilon}(\nabla f), r_{t}^{\prime}\right) d x-\varepsilon \int_{\Omega_{2}^{\varepsilon}}\left(\operatorname{div}\left(\left(A_{2} \nabla_{y} U\right)_{\varepsilon} f\right), r_{t}^{\prime}\right) d x \\
& =\varepsilon \int_{\Omega_{2}^{\varepsilon}}\left(\left(A_{2} \nabla_{y} U\right)_{\varepsilon}(\nabla f), r_{t}^{\prime}\right) d x+\varepsilon \int_{\Omega_{2}^{\varepsilon}}\left(\left(A_{2} \nabla_{y} U\right)_{\varepsilon} f, e\left(r_{t}^{\prime}\right)\right) d x
\end{aligned}
$$

поскольку $\left(\nu\left(A_{2} \nabla_{y} U\right)\right)_{\varepsilon}=0$ на $S_{2}^{\varepsilon}$ в соответствии с (4.26). Поэтому, учитывая гладкость $f$, неравенство (4.27) и повторяя доказательство (4.13), получаем

$$
\left|\int_{0}^{t} \int_{\Omega_{2}^{\varepsilon}}\left(\left(r_{2}^{0}\right)_{\varepsilon} f, r_{t}^{\prime}\right) d x d t\right| \leqslant C \varepsilon+\delta_{3} \mathscr{E}(r)
$$

Используя первое уравнение из (1.21) и (4.12), можно поменять в (4.19)-(4.28) индекс 2 на 1. Тогда с точностью до несушественных в (4.18) слагаемых интегралы по $\Omega_{1}^{\varepsilon}$ из (4.18) можно переписать в виде

$$
I_{1}=\int_{\Omega_{1}^{\varepsilon}}\left(\left(E_{1}^{0} R_{1}^{0}\right)_{\varepsilon} * f+\left(E_{1}^{0} a_{s}^{1}\right)_{\varepsilon} v_{s}-\left(E_{1}^{0} M_{s}^{1}\right)_{\varepsilon} *\left(v_{s}^{\prime \prime}\right), r_{t}^{\prime}\right) d x
$$

где $E_{1}^{0}=\left(m_{1} E_{1}\right) / M_{1}$ и $E_{1}=E$ на $\Omega_{1}^{\varepsilon}$ в соответствии с (1.16).

Рассмотрим теперь интеграл по $\Omega_{0}^{\varepsilon}$ из $(4.18)$, содержащий $u^{2}=u^{0}+u^{1}$. Из (1.6) и (4.15) непосредственно следует, что

$$
\begin{aligned}
& \int_{\Omega_{0}^{\varepsilon}}\left(A_{\varepsilon}^{\sigma} u^{1}, e\left(r_{t}^{\prime}\right)\right) d x=\frac{\sigma}{\varepsilon} \int_{\Omega_{0}^{\varepsilon}}\left(\left(A_{0} \nabla_{y} E_{s}\right)_{\varepsilon} v_{s}, e\left(r_{t}^{\prime}\right)\right) d x \\
& =\varepsilon \vartheta \int_{\Omega_{0}^{\varepsilon}}\left(\left(A_{0} \nabla_{y} E_{s}\right)_{\varepsilon} v_{s}, e\left(r_{t}^{\prime}\right)\right) d x+\varepsilon \omega \int_{\Omega_{0}^{\varepsilon}}\left(\left(A_{0} \nabla_{y} E_{s}\right)_{\varepsilon} v_{s}, e\left(r_{t}^{\prime}\right)\right) d x
\end{aligned}
$$

где $\omega=\sigma / \varepsilon^{2}-\vartheta$ и последний интеграл несущественен в (4.8). Действительно, интегрируя по частям, как и в (2.6)-(2.8), имеем

$$
\begin{aligned}
\mid \varepsilon \omega & \int_{0}^{t} \int_{\Omega_{0}^{\varepsilon}}\left(\left(A_{0} \nabla_{y} E_{s}\right)_{\varepsilon} v_{s}, e\left(r_{t}^{\prime}\right)\right) d x d t \mid \\
\leqslant & \varepsilon|\omega|\left|\int_{\Omega_{0}^{\varepsilon}}\left(\left(A_{0} \nabla_{y} E_{s}\right)_{\varepsilon} v_{s}, e(r)\right) d x+\int_{0}^{t} \int_{\Omega_{0}^{\varepsilon}}\left(\left(A_{0} \nabla_{y} E_{s}\right)_{\varepsilon} v_{s}^{\prime}, e(r)\right) d x d t\right| \\
\leqslant & \varepsilon|\omega|\|e(r)\|_{C^{0}\left([0, T] ; L^{2}\left(\Omega_{0}^{\varepsilon}\right)^{n \times n}\right)} \\
& \times\left(\left\|\left(A_{0} \nabla_{y} E_{s}\right)_{\varepsilon} v_{s}\right\|_{C^{0}\left([0, T] ; L^{2}\left(\Omega_{0}^{\varepsilon}\right)^{n \times n}\right)}+\left\|\left(A_{0} \nabla_{y} E_{s}\right)_{\varepsilon} v_{s}^{\prime}\right\|_{L^{1}\left(0, T ; L^{2}\left(\Omega_{0}^{\varepsilon}\right)^{n \times n}\right)}\right) \\
\leqslant & \frac{\varepsilon}{\sqrt{\sigma}}|\omega| C .
\end{aligned}
$$


Здесь учтены лемма 1 , первое неравенство из (4.2) для $E_{s}$ и гладкость $v_{s}$ для $s=1,2$. Кроме того, используя (1.6) и (4.15), получаем

$$
\begin{aligned}
\int_{\Omega_{0}^{\varepsilon}} & \left(A_{\varepsilon}^{\sigma} u^{0}, e\left(r_{t}^{\prime}\right)\right) d x \\
= & \frac{\sigma}{\varepsilon} \int_{\Omega_{0}^{\varepsilon}}\left(\left(A_{0} \nabla_{y} P\right)_{\varepsilon} * f, e\left(r_{t}^{\prime}\right)\right) d x-\frac{\sigma}{\varepsilon} \int_{\Omega_{0}^{\varepsilon}}\left(\left(A_{0} \nabla_{y} Q_{s}\right)_{\varepsilon} *\left(v_{s}^{\prime \prime}\right), e\left(r_{t}^{\prime}\right)\right) d x \\
= & \varepsilon \vartheta \int_{\Omega_{0}^{\varepsilon}}\left(\left(A_{0} \nabla_{y} P\right)_{\varepsilon} * f, e\left(r_{t}^{\prime}\right)\right) d x-\varepsilon \vartheta \int_{\Omega_{0}^{\varepsilon}}\left(\left(A_{0} \nabla_{y} Q_{s}\right)_{\varepsilon} *\left(v_{s}^{\prime \prime}\right), e\left(r_{t}^{\prime}\right)\right) d x \\
& +\varepsilon \omega \int_{\Omega_{0}^{\varepsilon}}\left(\left(A_{0} \nabla_{y} P\right)_{\varepsilon} * f, e\left(r_{t}^{\prime}\right)\right) d x-\varepsilon \omega \int_{\Omega_{0}^{\varepsilon}}\left(\left(A_{0} \nabla_{y} Q_{s}\right)_{\varepsilon} *\left(v_{s}^{\prime \prime}\right), e\left(r_{t}^{\prime}\right)\right) d x
\end{aligned}
$$

где последние два интеграла несущественны в (4.8). Действительно, как и в (4.31), имеем

$$
\begin{aligned}
& \left|\varepsilon \omega \int_{0}^{t} \int_{\Omega_{0}^{\varepsilon}}\left(\left(A_{0} \nabla_{y} P\right)_{\varepsilon} * f, e\left(r_{t}^{\prime}\right)\right) d x d t\right| \\
& \leqslant \varepsilon|\omega|\|e(r)\|_{C^{0}\left([0, T] ; L^{2}\left(\Omega_{0}^{\varepsilon}\right)^{n \times n}\right)} \\
& \quad \times\left(\left\|\left(A_{0} \nabla_{y} P\right)_{\varepsilon} * f\right\|_{C^{0}\left([0, T] ; L^{2}\left(\Omega_{0}^{\varepsilon}\right)^{n \times n}\right)}+\left\|\left(A_{0} \nabla_{y} P\right)_{\varepsilon} *\left(f_{t}^{\prime}\right)\right\|_{L^{1}\left(0, T ; L^{2}\left(\Omega_{0}^{\varepsilon}\right)^{n \times n}\right)}\right) \\
& \leqslant \frac{\varepsilon}{\sqrt{\sigma \vartheta}}|\omega| C,
\end{aligned}
$$

где учтены леммы 1 и 7 , неравенство (4.3) и гладкость $f$.

Для области $\Omega_{0}^{\varepsilon}$ также выполнена формула интегрирования по частям (4.19), но с обратным знаком у граничного интеграла, поскольку нормаль $\nu_{\varepsilon}$ является внутренней для гранищы $S_{0}^{\varepsilon}$ и внешней для границ $S_{1}^{\varepsilon}$ и $S_{2}^{\varepsilon}$. Поэтому, учитывая $(4.20)$, получаем

$$
\begin{aligned}
-\varepsilon \vartheta \int_{\Omega_{0}^{\varepsilon}}\left(\left(A_{0} \nabla_{y} E_{s}\right)_{\varepsilon} v_{s}, e\left(r_{t}^{\prime}\right)\right) d x \\
=\varepsilon \vartheta \int_{S_{0}^{\varepsilon}}\left(\left(\nu A_{0} \nabla_{y} E_{s}\right)_{\varepsilon} v_{s}, r_{t}^{\prime}\right) d s+\varepsilon \vartheta \int_{\Omega_{0}^{\varepsilon}}\left(\operatorname{div}\left(\left(A_{0} \nabla_{y} E_{s}\right)_{\varepsilon} v_{s}\right), r_{t}^{\prime}\right) d x \\
=\varepsilon \vartheta \int_{S_{0}^{\varepsilon}}\left(\left(\nu A_{0} \nabla_{y} E_{s}\right)_{\varepsilon} v_{s}, r_{t}^{\prime}\right) d s+\vartheta \int_{\Omega_{0}^{\varepsilon}}\left(\left(\operatorname{div}_{y} A_{0} \nabla_{y} E_{s}\right)_{\varepsilon} v_{s}, r_{t}^{\prime}\right) d x \\
\quad+\varepsilon \vartheta \int_{\Omega_{0}^{\varepsilon}}\left(\left(A_{0} \nabla_{y} E_{s}\right)_{\varepsilon} \nabla v_{s}, r_{t}^{\prime}\right) d x
\end{aligned}
$$

где предпоследний интеграл равен нулю (в силу (1.16), (1.18)), а вклад последнего интеграла в (4.18) оценивается величиной $\varepsilon \vartheta C$ (в силу первого неравенства из (4.2) для $E_{s}$, гладкости $v_{s}$ и леммы 1$)$. Кроме того, имеем

$$
\begin{aligned}
& -\varepsilon \vartheta \int_{\Omega_{0}^{\varepsilon}}\left(\left(A_{0} \nabla_{y} P\right)_{\varepsilon} * f, e\left(r_{t}^{\prime}\right)\right) d x \\
& =\varepsilon \vartheta \int_{S_{0}^{\varepsilon}}\left(\left(\nu A_{0} \nabla_{y} P\right)_{\varepsilon} * f, r_{t}^{\prime}\right) d s+\vartheta \int_{\Omega_{0}^{\varepsilon}}\left(\left(\operatorname{div}_{y} A_{0} \nabla_{y} P\right)_{\varepsilon} * f, r_{t}^{\prime}\right) d x \\
& \quad+\varepsilon \vartheta \int_{\Omega_{0}^{\varepsilon}}\left(\left(A_{0} \nabla_{y} P\right)_{\varepsilon} * \nabla f, r_{t}^{\prime}\right) d x
\end{aligned}
$$


где предпоследний интеграл сокращается с интегралом по $\Omega_{0}^{\varepsilon}$ из $(4.18)$, содержащим $f$ (в силу (1.19)), а вклад последнего интеграла в (4.18) оценивается величиной $\varepsilon \sqrt{\vartheta} C$ (в силу лемм 1,7 , неравенства (4.3) и гладкости $f$ ). Аналогичные (4.35) равенства выполнены для интеграла по $\Omega_{0}^{\varepsilon}$ из $(4.18)$, содержашего $\vartheta$ и $Q_{s}$ (см. (4.32)).

Таким образом, интегралы по $\Omega_{0}^{\varepsilon}$ заменяются в (4.18) на интегралы по $S_{0}^{\varepsilon}$ с точностью до несушественных слагаемых. Поэтому, используя (4.25), (4.29)-(4.35), неравенство (4.18) можно переписать в виде

$$
\begin{aligned}
I \leqslant & \int_{0}^{t}\left[\int_{\Omega_{1}^{\varepsilon}}\left(\left(E_{1}^{0} R_{1}^{0}\right)_{\varepsilon} * f+\left(E_{1}^{0} a_{s}^{1}\right)_{\varepsilon} v_{s}-\left(E_{1}^{0} M_{s}^{1}\right)_{\varepsilon} *\left(v_{s}^{\prime \prime}\right), r_{t}^{\prime}\right) d x\right. \\
& +\int_{\Omega_{2}^{\varepsilon}}\left(\left(E_{2}^{0} R_{2}^{0}\right)_{\varepsilon} * f, r_{t}^{\prime}\right) d x+\int_{\Omega_{2}^{\varepsilon}}\left(\left(E_{2}^{0} a_{s}^{2}\right)_{\varepsilon} v_{s}-\left(E_{2}^{0} M_{s}^{2}\right)_{\varepsilon} *\left(v_{s}^{\prime \prime}\right), r_{t}^{\prime}\right) d x \\
& +\varepsilon \vartheta \int_{S_{2}^{\varepsilon}}\left(\left(\nu A_{0} \nabla_{y} P\right)_{\varepsilon} * f, r_{t}^{\prime}\right) d s-\varepsilon \vartheta \int_{S_{0}^{\varepsilon}}\left(\left(\nu A_{0} \nabla_{y} Q_{s}\right)_{\varepsilon} *\left(v_{s}^{\prime \prime}\right), r_{t}^{\prime}\right) d s \\
& \left.+\varepsilon \vartheta \int_{S_{1}^{\varepsilon}}\left(\left(\nu A_{0} \nabla_{y} P\right)_{\varepsilon} * f, r_{t}^{\prime}\right) d s+\varepsilon \vartheta \int_{S_{0}^{\varepsilon}}\left(\left(\nu A_{0} \nabla_{y} E_{s}\right)_{\varepsilon} v_{s}, r_{t}^{\prime}\right) d s\right] d t \mid \\
& +C\left(\varepsilon(1+\vartheta+\sqrt{\vartheta})+\sigma+\frac{\varepsilon|\omega|}{\sqrt{\sigma}}+\frac{\varepsilon|\omega|}{\sqrt{\sigma \vartheta}}\right)+\left(\delta_{1}+\delta_{2}+\delta_{3}\right) \mathscr{E}(r),
\end{aligned}
$$

поскольку $S_{0}^{\varepsilon}=S_{1}^{\varepsilon} \cup S_{2}^{\varepsilon}$. Интегралы по $\Omega_{1}^{\varepsilon}$ и $\Omega_{2}^{\varepsilon}$ из (4.36) оцениваются одинаково: с точностью до несущественных слагаемых эти интегралы сокращаются с соответствующими граничными интегралами по $S_{1}^{\varepsilon}$ и $S_{2}^{\varepsilon}$. Поэтому рассмотрим, например, интеграл по $\Omega_{2}^{\varepsilon}$, содержащий матричнозначную функцию $R_{2}^{0}$. В определение этой функции входит вторая производная по $t$ от решений задачи (1.19). Непосредственно получить априорную оценку для этой производной достаточно сложно. Однако свертка по $t$ примечательна тем, что производные по $t$ можно “перебросить" на второй достаточно гладкий множитель, и возникшая проблема легко решается.

Напомним, что $\dot{P}$ обозначает такую первообразную по $t$ от $P$, что $\left.\dot{P}\right|_{t=0}=0$. Из равенств (2.20) и условий теоремы следует, что

$$
\begin{aligned}
R_{2}^{0} * f= & \left\langle m_{0} E_{2}^{T} P_{t t}^{\prime \prime}\right\rangle_{\mathbf{o}} * f=\left\langle m_{0} E_{2}^{T} P_{t}^{\prime}-E_{2}^{T} r_{0}\right\rangle_{\mathbf{o}} *\left(f_{t}^{\prime}\right) \\
& \left(\nu A_{0} \nabla_{y} P\right)_{\varepsilon} * f=\left(\nu A_{0} \nabla_{y} \dot{P}\right)_{\varepsilon} *\left(f_{t}^{\prime}\right)
\end{aligned}
$$

(см. (3.23)). Интегрируя по $t$ уравнение для $P$ из $(1.19)$, имеем

$$
m_{0} P_{t}^{\prime}-r_{0} E=\vartheta \operatorname{div}\left(A_{0} \nabla_{y} \dot{P}\right) \quad \text { в } Y_{0} \times(0, T) .
$$

По определению $P_{t}^{\prime}=\dot{P}_{t t}^{\prime \prime}$ и поэтому $\dot{P}$ удовлетворяет неравенству $(4.3)$ (см. доказательство леммы 1 ).

Из неравенств (4.3) для $P$ и $\dot{P}$ следует, что $W \equiv \vartheta A_{0} \nabla_{y} \dot{P} \in L^{2}\left(Y_{0}\right)^{n \times n \times n}$ и $\operatorname{div}_{y} W \in L^{2}\left(Y_{0}\right)^{n \times n}$ для всех $t \in[0, T]$. Поэтому для таких $t \in[0, T]$ определен след $\nu W$ на $S_{0}$ как элемент пространства $H_{P}^{-1 / 2}\left(S_{0}\right)^{n \times n}$ и

$$
\|\nu W\|_{H_{P}^{-1 / 2}\left(S_{0}\right)^{n \times n}}^{2} \leqslant C\left(\left\|\vartheta A_{0} \nabla_{y} \dot{P}\right\|_{L^{2}\left(Y_{0}\right)^{n \times n \times n}}^{2}+\left\|m_{0} P_{t}^{\prime}-r_{0} E\right\|_{L^{2}\left(Y_{0}\right)^{n \times n}}^{2}\right)
$$


в соответствии с неравенством (2.11). Тогда из леммы 4 следует, что

$$
\|\nu W\|_{C^{0}\left([0, T] ; H_{P}^{-1 / 2}\left(S_{0}\right)^{n \times n}\right)}^{2} \leqslant C(1+\vartheta) .
$$

Умножим (4.38) на $E_{2}^{T}$, проинтегрируем полученное соотношение по $Y_{0}$ и затем по частям. Тогда, учитывая (1.18) и (2.10), получаем

$$
\left\langle E_{2}^{T} r_{0}-m_{0} E_{2}^{T} P_{t}^{\prime}\right\rangle_{\mathbf{o}}=\vartheta \int_{S_{2}} E_{2}^{T} \nu\left(A_{0} \nabla_{y} \dot{P}\right) d s
$$

(см. (3.20)). Эти равенства гарантируют для всех $t \in[0, T]$ разрешимость задачи Неймана на $Y_{2}$ :

$$
\begin{gathered}
-\operatorname{div}_{y}\left(A_{2} \nabla_{y} V\right)=E_{2}^{0}\left\langle E_{2}^{T} r_{0}-m_{0} E_{2}^{T} P_{t}^{\prime}\right\rangle_{\text {o }} \quad \text { в } Y_{2}, \\
-\nu\left(A_{2} \nabla_{y} V\right)=\vartheta \nu\left(A_{0} \nabla_{y} \dot{P}\right) \text { на } S_{2} .
\end{gathered}
$$

В силу леммы 3 для всех $t \in[0, T]$ существует 1-периодическое решение $V \in\left(H_{P}^{1}\left(Y_{2}\right)^{n} \cap L^{2}\left(Y_{2}\right)^{n} / \Re\right)^{n}$ задачи (4.40) и

$$
\begin{aligned}
& \left\|A_{2} \nabla_{y} V\right\|_{L^{2}\left(Y_{2}\right)^{n \times n \times n}} \\
& \quad \leqslant C\left(\left\|\vartheta \nu\left(A_{0} \nabla_{y} \dot{P}\right)\right\|_{H_{P}^{-1 / 2}\left(S_{2}\right)^{n \times n}}+\left\|E_{2}^{0}\left\langle E_{2}^{T} r_{0}-m_{0} E_{2}^{T} P_{t}^{\prime}\right\rangle_{\mathbf{o}}\right\|_{L^{2}\left(Y_{2}\right)^{n \times n}}\right) .
\end{aligned}
$$

Следовательно, используя (4.2), (4.3) и (4.39), имеем

$$
\left\|\left(A_{2} \nabla_{y} V\right)_{\varepsilon}\right\|_{C^{0}\left([0, T] ; L^{2}\left(\Omega_{2}^{\varepsilon}\right)^{n \times n \times n}\right)} \leqslant C \sqrt{1+\vartheta} \leqslant C(1+\sqrt{\vartheta}),
$$

поскольку область $\Omega_{2}^{\varepsilon} \subset \Omega$ ограничена.

Таким образом, используя (4.19), (4.20), (4.37) и (4.40), получаем

$$
\begin{aligned}
- & \int_{\Omega_{2}^{\varepsilon}}\left(\left(E_{2}^{0} R_{2}^{0}\right)_{\varepsilon} * f, r_{t}^{\prime}\right) d x=-\int_{\Omega_{2}^{\varepsilon}}\left(\left(\operatorname{div}_{y} A_{2} \nabla_{y} V\right)_{\varepsilon} *\left(f_{t}^{\prime}\right), r_{t}^{\prime}\right) d x \\
= & \varepsilon \int_{\Omega_{2}^{\varepsilon}}\left(\left(A_{2} \nabla_{y} V\right)_{\varepsilon} *\left(\nabla f_{t}^{\prime}\right), r_{t}^{\prime}\right) d x-\varepsilon \int_{\Omega_{2}^{\varepsilon}}\left(\operatorname{div}\left(\left(A_{2} \nabla_{y} V\right)_{\varepsilon} *\left(f_{t}^{\prime}\right)\right), r_{t}^{\prime}\right) d x \\
= & \varepsilon \int_{\Omega_{2}^{\varepsilon}}\left(\left(A_{2} \nabla_{y} V\right)_{\varepsilon} *\left(\nabla f_{t}^{\prime}\right), r_{t}^{\prime}\right) d x+\varepsilon \int_{\Omega_{2}^{\varepsilon}}\left(\left(A_{2} \nabla_{y} V\right)_{\varepsilon} *\left(f_{t}^{\prime}\right), e\left(r_{t}^{\prime}\right)\right) d x \\
& -\varepsilon \int_{S_{2}^{\varepsilon}}\left(\left(\nu A_{2} \nabla_{y} V\right)_{\varepsilon} *\left(f_{t}^{\prime}\right), r_{t}^{\prime}\right) d s \\
= & \varepsilon \vartheta \int_{S_{2}^{\varepsilon}}\left(\left(\nu A_{0} \nabla_{y} \dot{P}\right)_{\varepsilon} *\left(f_{t}^{\prime}\right), r_{t}^{\prime}\right) d s+\varepsilon \int_{\Omega_{2}^{\varepsilon}}\left(\left(A_{2} \nabla_{y} V\right)_{\varepsilon} *\left(\nabla f_{t}^{\prime}\right), r_{t}^{\prime}\right) d x \\
& +\varepsilon \int_{\Omega_{2}^{\varepsilon}}\left(\left(A_{2} \nabla_{y} V\right)_{\varepsilon} *\left(f_{t}^{\prime}\right), e\left(r_{t}^{\prime}\right)\right) d x,
\end{aligned}
$$

где последние два интеграла несущественны в (4.36) (в силу лемм 1 и 7 , гладкости $f$ и (4.41)), а граничный интеграл сокращается с соответствующим граничным интегралом из (4.36) в силу (4.37). Оценивая аналогичным образом оставшшеся в (4.36) интегралы и выбирая подходящие $\delta_{1}, \ldots, \delta_{l}$, заключаем, что

$$
\mathscr{E}(r) \leqslant C\left(\varepsilon(1+\vartheta+\sqrt{\vartheta})+\sigma+\frac{\varepsilon}{\sqrt{\sigma}}|\omega|+\frac{\varepsilon}{\sqrt{\sigma \vartheta}}|\omega|\right) .
$$

Из условия (1.2) следует, что $\varepsilon / \sqrt{\sigma} \rightarrow 1 / \sqrt{\vartheta}$ при $\varepsilon \rightarrow 0$. Поэтому при $\varepsilon \rightarrow 0$ величина $\varepsilon / \sqrt{\sigma}$ ограничена, что доказьвает теорему 2 . 


\section{§5. Доказательство теоремы 1}

В случае, когда выполнено условие (1.1), начальные члены асимптотического разложения решения задачи (1.4) определим равенством (3.47). Определим также подправленную асимптотику $v_{a}$ равенством

$$
\begin{aligned}
v_{a}=v+\frac{\varepsilon^{2}}{\sigma} E_{2 \varepsilon} v_{2} & +\frac{\varepsilon^{2}}{\sigma} p_{\varepsilon} f-\frac{\varepsilon^{2}}{\sigma} q_{\varepsilon} v_{t t}^{\prime \prime} \\
& +\varepsilon\left(N_{1 a}\right)_{\varepsilon} \eta \nabla v+\varepsilon\left(N_{1 b}\right)_{\varepsilon} g+\varepsilon\left(N_{2 a}^{1}\right)_{\varepsilon} \eta \nabla v+\varepsilon\left(N_{2 b}\right)_{\varepsilon} g
\end{aligned}
$$

(см. $\S 4)$. Здесь $v$ является решением задачи (1.15) и $v_{2}$ удовлетворяет соотношениям (3.44) и (3.45). Кроме того, выполнены неравенства (4.2) и аналогичные неравенства для матричнозначных функций $E_{2}, N_{1 b}, N_{2 a}^{1}, N_{2 b}, p$ и $q$. Напомним, что эти функции определяются в (1.11), (1.13), (1.18), (2.15), (3.11), (3.41) и (3.9), где $E_{2}$ заменено на $E_{2}^{1}$.

Предположим сначала, что $w_{0}=0$ и $\left.g\right|_{t=0}=0$. Пусть $u$ является решением задачи (1.4). Введем обозначения (4.5). Тогда вьполнено неравенство (4.6) и $\left.r_{a}\right|_{t=0}=0$ в соответствии с предположениями на начальные данные.

В силу представления (5.1) и первого неравенства из (4.2) имеем

$$
\left\|\left.r_{t}^{\prime}\right|_{t=0}\right\|_{L^{2}(\Omega)^{n}}^{2} \leqslant C\left(\varepsilon^{2}+\frac{\varepsilon^{2}}{\sigma}\right) .
$$

Кроме того, $\left.r\right|_{t=0}=\left.\left(\varepsilon^{2} / \sigma\right) q_{\varepsilon} v_{t t}^{\prime \prime}\right|_{t=0}-\left.\left(\varepsilon^{2} / \sigma\right) p_{\varepsilon} f\right|_{t=0}$ и эта вектор-функция имеет нулевые компоненты на $\bar{\Omega}_{12}^{\varepsilon}$. Следовательно, используя формулу дифференцирования (4.4) и неравенство (4.2) для $q$ и $p$, получаем

$$
\sigma\left\|e\left(\left.r\right|_{t=0}\right)\right\|_{L^{2}\left(\Omega_{0}^{\varepsilon}\right)^{n \times n}}^{2}+\left\|e\left(\left.r\right|_{t=0}\right)\right\|_{L^{2}\left(\Omega_{12}^{\varepsilon}\right)^{n \times n}}^{2} \leqslant C\left(\varepsilon^{2}+\frac{\varepsilon^{2}}{\sigma}\right) .
$$

Таким образом, учитьвая доказательство леммы 1 и неравенства (5.2), (5.3), для доказательства теоремы 1 достаточно получить неравенство

$$
I \leqslant \frac{1}{2} \mathscr{E}(r)+C\left(\varepsilon+\sigma+\frac{\varepsilon^{2}}{\sigma}\right)
$$

где $I$ определено в (4.7) и постоянная $C$ не зависит от $\varepsilon$ и $\sigma$.

Используя равенства (3.16) и (4.4), имеем

$$
\begin{aligned}
\nabla v_{a}= & \nabla r_{a}+\left(E_{1}+\nabla_{y} N_{1 a}\right)_{\varepsilon} \nabla v+\left(\nabla_{y} N_{1 b}\right)_{\varepsilon} \nabla g \\
& +\left(E_{2}^{1}+\nabla_{y} N_{2 a}^{1}\right)_{\varepsilon} \nabla v+\left(\nabla_{y} N_{2 b}\right)_{\varepsilon} \nabla g+\frac{\varepsilon}{\sigma}\left(\nabla_{y} E_{2}\right)_{\varepsilon} v_{2} \\
& +\frac{\varepsilon}{\sigma}\left(\nabla_{y} p\right)_{\varepsilon} f-\frac{\varepsilon}{\sigma}\left(\nabla_{y} q\right)_{\varepsilon} v_{t t}^{\prime \prime}+\varepsilon J_{1}+\frac{\varepsilon^{2}}{\sigma} J_{2},
\end{aligned}
$$

где интегралы по $\Omega \times(0, t)$ из $(4.7)$, содержащие $\nabla r_{a}, J_{1}$ и $J_{2}$, несущественны в $(5.4)$ (см., например, (4.13)).

Определим $\widetilde{A}_{2 a}(y)=A_{2} E_{2}^{1}+A_{2} \nabla_{y} N_{2 a}^{1}$ и $\widetilde{A}_{2 b}(y)=B_{2}+A_{2} \nabla_{y} N_{2 b}$. Тогда выполнены соотношения (4.11), в которых $\widehat{A}_{2}$ следует заменить на $\widehat{A}_{2}^{1}=\left\langle E_{2}^{T} \widetilde{A}_{2 a}\right\rangle_{2}$ (равное нулю в силу $(3.15)$ ), и равенство

$$
A_{\varepsilon}^{\sigma} \nabla v_{a}+B_{\varepsilon}^{\sigma} g=A_{\varepsilon}^{\sigma} \nabla r_{a}+\varepsilon A_{\varepsilon}^{\sigma} J_{1}+\left(\widetilde{A}_{2 a}\right)_{\varepsilon} \nabla v+\left(\widetilde{A}_{2 b}\right)_{\varepsilon} g \text { в } \Omega_{2}^{\varepsilon} .
$$


Аналогично получаем равенство (4.12), в котором $v_{1}$ заменено на $v$. Кроме того, равенства (4.14) в рассматриваемом случае заменяются на равенства

$$
\begin{gathered}
\left(v_{a}\right)_{t t}^{\prime \prime}=v_{t t}^{\prime \prime}+\varepsilon J_{3}+\frac{\varepsilon^{2}}{\sigma} J_{4} \text { в } \Omega_{0}^{\varepsilon}, \\
\left(v_{a}\right)_{t t}^{\prime \prime}=E_{1 \varepsilon} v_{t t}^{\prime \prime}+\varepsilon J_{3} \text { в } \Omega_{1}^{\varepsilon}, \quad\left(v_{a}\right)_{t t}^{\prime \prime}=E_{2 \varepsilon}^{1} v_{t t}^{\prime \prime}+\varepsilon J_{3} \text { в } \Omega_{2}^{\varepsilon},
\end{gathered}
$$

где соответствующие интегралы с $J_{3}$ и $J_{4}$ несущественны в (5.4).

Выделяя сингулярные слагаемые в (5.5), введем обозначения

$$
\begin{gathered}
u^{0}=\frac{\varepsilon}{\sigma}\left(\nabla_{y} p\right)_{\varepsilon} f-\frac{\varepsilon}{\sigma}\left(\nabla_{y} q\right)_{\varepsilon} v_{t t}^{\prime \prime}, \\
u^{1}=\frac{\varepsilon}{\sigma}\left(\nabla_{y} E_{2}\right)_{\varepsilon} v_{2}, \quad u^{2}=u^{0}+u^{1}
\end{gathered}
$$

и определим $u^{3}$ из (4.16). Тогда выполнено (4.17). Следовательно, интегралы по $\Omega_{0}^{\varepsilon} \times(0, t)$ из $(4.7)$, содержащие $A_{\sigma}^{\varepsilon} u^{3}$ и $B_{\sigma}^{\varepsilon} g$, несущественны в $(5.4)$.

Таким образом, учитывая (4.10)-(4.13), (4.17), (5.5)-(5.8) в (4.7), имеем

$$
\begin{aligned}
I \leqslant & \int_{0}^{t}\left[\int_{\Omega_{0}^{\varepsilon}}\left(\left(r_{0}\right)_{\varepsilon} f, r_{t}^{\prime}\right) d x-\int_{\Omega_{0}^{\varepsilon}}\left(\left(m_{0}\right)_{\varepsilon} v_{t t}^{\prime \prime}, r_{t}^{\prime}\right) d x\right. \\
& -\int_{\Omega_{0}^{\varepsilon}}\left(A_{\varepsilon}^{\sigma} u^{2}, e\left(r_{t}^{\prime}\right)\right) d x-\int_{\Omega_{2}^{\varepsilon}}\left(\left(\widetilde{A}_{2 a}\right)_{\varepsilon} \nabla v+\left(\widetilde{A}_{2 b}\right)_{\varepsilon} g, e\left(r_{t}^{\prime}\right)\right) d x \\
& +\int_{\Omega_{2}^{\varepsilon}}\left(\left(r_{2}\right)_{\varepsilon} f, r_{t}^{\prime}\right) d x-\int_{\Omega_{2}^{\varepsilon}}\left(\left(m_{2} E_{2}^{1}\right)_{\varepsilon} v_{t t}^{\prime \prime}, r_{t}^{\prime}\right) d x+\int_{\Omega_{1}^{\varepsilon}}\left(\left(r_{1}\right)_{\varepsilon} f, r_{t}^{\prime}\right) d x \\
& \left.-\int_{\Omega_{1}^{\varepsilon}}\left(\left(m_{1} E_{1}\right)_{\varepsilon} v_{t t}^{\prime \prime}, r_{t}^{\prime}\right) d x-\int_{\Omega_{1}^{\varepsilon}}\left(\left(\widetilde{A}_{1 a}\right)_{\varepsilon} \nabla v+\left(\widetilde{A}_{1 b}\right)_{\varepsilon} g, e\left(r_{t}^{\prime}\right)\right) d x\right] d t \\
& +C\left(\varepsilon+\sigma+\frac{\varepsilon^{2}}{\sigma}\right)+\left(\delta_{1}+\delta_{2}\right) \mathscr{E}(r) .
\end{aligned}
$$

Обозначим интегралы по $\Omega_{2}^{\varepsilon}$ из (5.9) через $I_{2}$. Тогда, используя (4.11), (4.19) и (4.20), получаем

$$
I_{2}=\int_{\Omega_{2}^{\varepsilon}}\left(\left(r_{2}\right)_{\varepsilon} f-\left(m_{2} E_{2}^{1}\right)_{\varepsilon} v_{t t}^{\prime \prime}, r_{t}^{\prime}\right) d x-\int_{\Omega_{2}^{\varepsilon}}\left(\left(\widetilde{A}_{2 a}\right)_{\varepsilon} \nabla^{2} v+\left(\widetilde{A}_{2 b}\right)_{\varepsilon} \nabla g, r_{t}^{\prime}\right) d x
$$

Определим $E_{2}^{0}(y)=\left(m_{2}(y) E_{2}(y)\right) / M_{2}$. Введем также обозначения (4.22), в которых $\widehat{A}_{2}$ следует заменить на $\widehat{A}_{2}^{1}$, для матричнозначных функций, удовлетворяюших соотношениям (4.23). Напомнив, что $\widehat{A}_{2}^{1}=0$ в силу (3.15), перепишем равенство (3.44) в виде

$$
M_{2} v_{t t}^{\prime \prime}+\widehat{m}_{2}^{0} v_{t t}^{\prime \prime}-\widehat{A}_{2}^{1} \nabla^{2} v+\widehat{a}_{2}^{2} v_{2}-R_{2} f-\widehat{r}_{2}^{0} f-\widetilde{B}_{2} \nabla g=0
$$

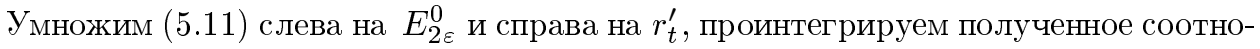
шение по $\Omega_{2}^{\varepsilon}$ и учтем результат в (5.10). Тогда, используя $(4.22)$, равенство $(5.10)$ можно переписать в виде

$$
\begin{aligned}
I_{2} & =\int_{\Omega_{2}^{\varepsilon}}\left(\left(E_{2}^{0} \widehat{m}_{2}^{0}\right)_{\varepsilon} v_{t t}^{\prime \prime}+\left(E_{2}^{0} \widehat{a}_{2}^{2}\right)_{\varepsilon} v_{2}-\left(E_{2}^{0} \widehat{r}_{2}^{0}\right)_{\varepsilon} f, r_{t}^{\prime}\right) d x \\
& +\int_{\Omega_{2}^{\varepsilon}}\left(\left(r_{2}^{0}\right)_{\varepsilon} f, r_{t}^{\prime}\right) d x-\int_{\Omega_{2}^{\varepsilon}}\left(\left(A_{2 a}^{0}\right)_{\varepsilon} \nabla^{2} v+\left(A_{2 b}^{0}\right)_{\varepsilon} \nabla g+\left(m_{2}^{0}\right)_{\varepsilon} v_{t t}^{\prime \prime}, r_{t}^{\prime}\right) d x .
\end{aligned}
$$


В силу (4.26)-(4.28) последние два интеграла из (5.12) несущественны в (5.9).

Аналогично с точностью до несушественных в (5.9) слагаемых интегралы по $\Omega_{1}^{\varepsilon}$ из (5.9) можно переписать в виде

$$
I_{1}=\int_{\Omega_{1}^{\varepsilon}}\left(\left(E_{1}^{0} \widehat{m}_{1}^{0}\right)_{\varepsilon} v_{t t}^{\prime \prime}+\left(E_{1}^{0} \widehat{a}_{2}^{1}\right)_{\varepsilon} v_{2}-\left(E_{1}^{0} \widehat{r}_{1}^{0}\right)_{\varepsilon} f, r_{t}^{\prime}\right) d x
$$

где $E_{1}^{0}=\left(m_{1} E_{1}\right) / M_{1}$ и использовано равенство $(3.45)$.

Рассмотрим теперь интеграл по $\Omega_{0}^{\varepsilon}$ из $(5.9)$, содержаший $u^{2}$. Непосредственно из (1.6) и (5.8) следует, что

$$
\begin{aligned}
\int_{\Omega_{0}^{\varepsilon}}\left(A_{\varepsilon}^{\sigma} u^{2}, e\left(r_{t}^{\prime}\right)\right) d x=\varepsilon \int_{\Omega_{0}^{\varepsilon}}\left(\left(A_{0} \nabla_{y} E_{2}\right)_{\varepsilon} v_{2}, e\left(r_{t}^{\prime}\right)\right) d x \\
\quad+\varepsilon \int_{\Omega_{0}^{\varepsilon}}\left(\left(A_{0} \nabla_{y} p\right)_{\varepsilon} f, e\left(r_{t}^{\prime}\right)\right) d x-\varepsilon \int_{\Omega_{0}^{\varepsilon}}\left(\left(A_{0} \nabla_{y} q\right)_{\varepsilon} v_{t t}^{\prime \prime}, e\left(r_{t}^{\prime}\right)\right) d x
\end{aligned}
$$

Таким образом, как и в (4.34), получаем

$$
\begin{aligned}
& -\varepsilon \int_{\Omega_{0}^{\varepsilon}}\left(\left(A_{0} \nabla_{y} E_{2}\right)_{\varepsilon} v_{2}, e\left(r_{t}^{\prime}\right)\right) d x=\varepsilon \int_{S_{0}^{\varepsilon}}\left(\left(\nu A_{0} \nabla_{y} E_{2}\right)_{\varepsilon} v_{2}, r_{t}^{\prime}\right) d s \\
& \quad+\int_{\Omega_{0}^{\varepsilon}}\left(\left(\operatorname{div}_{y} A_{0} \nabla_{y} E_{2}\right)_{\varepsilon} v_{2}, r_{t}^{\prime}\right) d x+\varepsilon \int_{\Omega_{0}^{\varepsilon}}\left(\left(A_{0} \nabla_{y} E_{2}\right)_{\varepsilon} \nabla v_{2}, r_{t}^{\prime}\right) d x
\end{aligned}
$$

где предпоследний интеграл равен нулю (в силу (1.18)), а вклад последнего интеграла в (5.9) оценивается величиной $\varepsilon C$ (в силу первого неравенства из $(4.2)$ для $E_{2}$, гладкости $v_{2}$ и леммы 1$)$. Кроме того, имеем

$$
\begin{aligned}
& -\varepsilon \int_{\Omega_{0}^{\varepsilon}}\left(\left(A_{0} \nabla_{y} p\right)_{\varepsilon} f, e\left(r_{t}^{\prime}\right)\right) d x=\varepsilon \int_{S_{0}^{\varepsilon}}\left(\left(\nu A_{0} \nabla_{y} p\right)_{\varepsilon} f, r_{t}^{\prime}\right) d s \\
& \quad+\int_{\Omega_{0}^{\varepsilon}}\left(\left(\operatorname{div}_{y} A_{0} \nabla_{y} p\right)_{\varepsilon} f, r_{t}^{\prime}\right) d x+\varepsilon \int_{\Omega_{0}^{\varepsilon}}\left(\left(A_{0} \nabla_{y} p\right)_{\varepsilon} \nabla f, r_{t}^{\prime}\right) d x
\end{aligned}
$$

где предпоследний интеграл сокращается с интегралом по $\Omega_{0}^{\varepsilon}$ из $(5.9)$, содержащим $f$ (в силу (2.15)), а вклад последнего интеграла в (5.9) оценивается величиной $\varepsilon C$ (в силу леммы 1, неравенства (4.2) и гладкости $f$ ). Аналогичное (5.16) равенство выполнено для интеграла по $\Omega_{0}^{\varepsilon}$ из (5.14), содержащего $q$.

Таким образом, интегралы по $\Omega_{0}^{\varepsilon}$ заменяются в $(5.9)$ на интегралы по $S_{0}^{\varepsilon}$ с точностью до несущественных слагаемых. Поэтому, используя (5.12)-(5.16), неравенство (5.9) можно переписать в виде

$$
\begin{aligned}
I \leqslant & \int_{0}^{t}\left[\int_{\Omega_{1}^{\varepsilon}}\left(\left(E_{1}^{0} \widehat{m}_{1}^{0}\right)_{\varepsilon} v_{t t}^{\prime \prime}+\left(E_{1}^{0} \widehat{a}_{2}^{1}\right)_{\varepsilon} v_{2}-\left(E_{1}^{0} \widehat{r}_{1}^{0}\right)_{\varepsilon} f, r_{t}^{\prime}\right) d x\right. \\
& -\int_{\Omega_{2}^{\varepsilon}}\left(\left(E_{2}^{0} \widehat{r}_{2}^{0}\right)_{\varepsilon} f, r_{t}^{\prime}\right) d x+\int_{\Omega_{2}^{\varepsilon}}\left(\left(E_{2}^{0} \widehat{a}_{2}^{2}\right)_{\varepsilon} v_{2}+\left(E_{2}^{0} \widehat{m}_{2}^{0}\right)_{\varepsilon} v_{t t}^{\prime \prime}, r_{t}^{\prime}\right) d x \\
& +\varepsilon \int_{S_{2}^{\varepsilon}}\left(\left(\nu A_{0} \nabla_{y} p\right)_{\varepsilon} f, r_{t}^{\prime}\right) d s-\varepsilon \int_{S_{0}^{\varepsilon}}\left(\left(\nu A_{0} \nabla_{y} q\right)_{\varepsilon} v_{t t}^{\prime \prime}, r_{t}^{\prime}\right) d s \\
& \left.+\varepsilon \int_{S_{1}^{\varepsilon}}\left(\left(\nu A_{0} \nabla_{y} p\right)_{\varepsilon} f, r_{t}^{\prime}\right) d s+\varepsilon \int_{S_{0}^{\varepsilon}}\left(\left(\nu A_{0} \nabla_{y} E_{2}\right)_{\varepsilon} v_{2}, r_{t}^{\prime}\right) d s\right] d t \mid \\
& +C\left(\varepsilon+\sigma+\frac{\varepsilon^{2}}{\sigma}\right)+\left(\delta_{1}+\delta_{2}+\delta_{3}\right) \mathscr{E}(r)
\end{aligned}
$$


поскольку $S_{0}^{\varepsilon}=S_{1}^{\varepsilon} \cup S_{2}^{\varepsilon}$. Интегралы по $\Omega_{1}^{\varepsilon}$ и $\Omega_{2}^{\varepsilon}$ из (5.17) оцениваются одинаково: с точностью до несушественных слагаемых эти интегралы сокрашаются с соответствующими граничными интегралами по $S_{1}^{\varepsilon}$ и $S_{2}^{\varepsilon}$. Поэтому рассмотрим, например, интеграл по $\Omega_{2}^{\varepsilon}$, содержащий $E_{2}^{0} \widehat{r}_{2}^{0}$.

Обозначим $W=A_{0} \nabla_{y} p$. Тогда $W \in L^{2}\left(Y_{0}\right)^{n \times n \times n}$ и $\operatorname{div}_{y} W \in L^{2}\left(Y_{0}\right)^{n \times n}$, поскольку $p \in H_{P}^{1}\left(Y_{0}\right)^{n \times n}$ удовлетворяет первому уравнению из (2.15). Поэтому определен след $\nu W$ на $S_{0}$ как элемент пространства $H_{P}^{-1 / 2}\left(S_{0}\right)^{n \times n}$ в соответствии с неравенством (2.11). Учитывая (1.17) и (3.30), получаем

$$
\left\langle E_{2}^{T} E_{2}^{0} \widehat{r}_{2}^{0}\right\rangle_{\mathrm{o}}=\widehat{r}_{2}^{0}=\int_{S_{2}} E_{2}^{T} \nu\left(A_{0} \nabla_{y} p\right) d s .
$$

Эти равенства гарантируют разрешимость 1-периодической задачи на $Y_{2}$ :

$$
-\operatorname{div}_{y}\left(A_{2} \nabla_{y} U\right)=E_{2}^{0} \widehat{r}_{2}^{0} \text { в } Y_{2}, \quad-\nu\left(A_{2} \nabla_{y} U\right)=\nu\left(A_{0} \nabla_{y} p\right) \text { на } S_{2} \text {. }
$$

В силу леммы 3 решение этой задачи существует и удовлетворяет (4.27).

Таким образом, используя (4.19), (4.20) и (5.18), получаем

$$
\begin{aligned}
\int_{\Omega_{2}^{\varepsilon}} & \left(\left(E_{2}^{0} \widehat{r}_{2}^{0}\right)_{\varepsilon} f, r_{t}^{\prime}\right) d x=-\int_{\Omega_{2}^{\varepsilon}}\left(\left(\operatorname{div}_{y} A_{2} \nabla_{y} U\right)_{\varepsilon} f, r_{t}^{\prime}\right) d x \\
= & \varepsilon \int_{\Omega_{2}^{\varepsilon}}\left(\left(A_{2} \nabla_{y} U\right)_{\varepsilon} \nabla f, r_{t}^{\prime}\right) d x-\varepsilon \int_{\Omega_{2}^{\varepsilon}}\left(\operatorname{div}\left(\left(A_{2} \nabla_{y} U\right)_{\varepsilon} f\right), r_{t}^{\prime}\right) d x \\
= & \varepsilon \int_{\Omega_{2}^{\varepsilon}}\left(\left(A_{2} \nabla_{y} U\right)_{\varepsilon} \nabla f, r_{t}^{\prime}\right) d x+\varepsilon \int_{\Omega_{2}^{\varepsilon}}\left(\left(A_{2} \nabla_{y} U\right)_{\varepsilon} f, e\left(r_{t}^{\prime}\right)\right) d x \\
& -\varepsilon \int_{S_{2}^{\varepsilon}}\left(\left(\nu A_{2} \nabla_{y} U\right)_{\varepsilon} f, r_{t}^{\prime}\right) d s \\
= & \varepsilon \int_{S_{2}^{\varepsilon}}\left(\left(\nu A_{0} \nabla_{y} p\right)_{\varepsilon} f, r_{t}^{\prime}\right) d s+\varepsilon \int_{\Omega_{2}^{\varepsilon}}\left(\left(A_{2} \nabla_{y} U\right)_{\varepsilon} \nabla f, r_{t}^{\prime}\right) d x \\
& +\varepsilon \int_{\Omega_{2}^{\varepsilon}}\left(\left(A_{2} \nabla_{y} U\right)_{\varepsilon} f, e\left(r_{t}^{\prime}\right)\right) d x
\end{aligned}
$$

где последние два интеграла несушественны в (5.17) (в силу леммы 1, гладкости $f$ и неравенства (4.27)), а граничный интеграл сокращается с соответствующим граничньм интегралом из (5.17), содержащим $f$. Оценивая аналогичньм образом оставшиеся в (5.17) интегралы и выбирая соответствующие $\delta_{1}, \ldots, \delta_{l}$, заключаем, что

$$
\mathscr{E}(r) \leqslant C\left(\varepsilon+\sigma+\frac{\varepsilon^{2}}{\sigma}\right)
$$

Будем обозначать через $\dot{u}, \dot{f}, \dot{g}, \dot{v}$ первообразные по $t$ от вектор-функций $u, f$, $g, v$ из (1.4) и (1.15). Тогда $\dot{u}$ является решением задачи (1.4) с правой частью $r_{\varepsilon} \dot{f}+m_{\varepsilon} w_{1}+\operatorname{div}\left(B_{\sigma}^{\varepsilon} \dot{g}\right)$ и $\left.\dot{u}\right|_{t=0}=0$. Для такой задачи уже доказано неравенство (5.20), и поэтому

$$
\|u-v\|_{C^{0}\left([0, T] ; L^{2}(\Omega)^{n}\right)}^{2}=\left\|\dot{u}_{t}^{\prime}-\dot{v}_{t}^{\prime}\right\|_{C^{0}\left([0, T] ; L^{2}(\Omega)^{n}\right)}^{2} \leqslant C\left(\varepsilon+\sigma+\frac{\varepsilon^{2}}{\sigma}\right)
$$

что завершает доказательство теоремы 1. 


\section{§ 6. Доказательство теоремы 3}

Из условия (1.3) следует, что $\varkappa \equiv \sqrt{\sigma} / \varepsilon$ стремится к нулю при $\varepsilon \rightarrow 0$. Определим $P, Q_{1}$ и $Q_{2}$ как 1-периодические решения начально-краевых задач (1.19), в которых $\vartheta$ заменено на $\varkappa^{2}$. Пусть $v_{1}, v_{2}$ являются решениями задачи $(1.22)$ и подправленная асимптотика $v_{a}$ задана равенством (4.1). Как и в $\S 5$, предположим сначала, что $w_{0}=0$ и $\left.g\right|_{t=0}=0$. Введем обозначения (4.5). Тогда $\left.r\right|_{t=0}=0$. Кроме того, из (2.20) и (4.1) получаем

$$
\left\|\left.r_{t}^{\prime}\right|_{t=0}\right\|_{L^{2}(\Omega)^{n}} \leqslant C \varepsilon
$$

Таким образом, заменяя $\vartheta$ на $\varkappa^{2}$ в соответствующих формулах из $\S 4$, для доказательства теоремы 3 можно повторить доказательство теоремы 2 (по крайней мере, до неравенства (4.36)). Задача (1.22) совпадает с задачей (1.21), в которой $M_{s}^{l}=0, a_{s}^{l}=0$ и $R_{s}^{0}=0$ для $s, l=1,2$. Поэтому в соответствии с рассуждениями из $\S 4$ неравенство (4.36) принимает вид

$$
\begin{aligned}
I \leqslant & \mid \int_{0}^{t}\left[\varepsilon \varkappa^{2} \int_{S_{0}^{\varepsilon}}\left(\left(\nu A_{0} \nabla_{y} P\right)_{\varepsilon} * f, r_{t}^{\prime}\right) d s\right. \\
& \left.-\varepsilon \varkappa^{2} \int_{S_{0}^{\varepsilon}}\left(\left(\nu A_{0} \nabla_{y} Q_{s}\right)_{\varepsilon} *\left(v_{s}^{\prime \prime}\right), r_{t}^{\prime}\right) d s+\varepsilon \varkappa^{2} \int_{S_{0}^{\varepsilon}}\left(\left(\nu A_{0} \nabla_{y} E_{s}\right)_{\varepsilon} v_{s}, r_{t}^{\prime}\right) d s\right] d t \mid \\
& +C\left(\varepsilon\left(1+\varkappa^{2}+\varkappa\right)+\sigma\right)+\left(\delta_{1}+\delta_{2}+\delta_{3}\right) \mathscr{E}(r) .
\end{aligned}
$$

Напомним, что $\omega=\sigma / \varepsilon^{2}-\varkappa^{2}$ в обозначениях из $\S 4$ и поэтому $\omega=0$ в (4.36).

Для $s=1,2$ из неравенств Коши-Буняковского и (4.2) получаем

$$
\left|\left\langle m_{0} E_{s}^{T}\left(P_{t}^{\prime}-\frac{r_{0}}{m_{0}} E\right)\right\rangle_{\mathbf{o}}\right| \leqslant C\left\|P_{t}^{\prime}-\frac{r_{0}}{m_{0}} E\right\|_{L^{2}\left(Y_{0}\right)^{n \times n}},
$$

и поэтому $\left\|\left\langle m_{0} E_{s}^{T}\left(P_{t}^{\prime}-\left(r_{0} / m_{0}\right) E\right)\right\rangle_{\mathbf{o}}\right\|_{C^{0}([0, T])^{n \times n}} \leqslant C \sqrt{\varkappa}$ с силу леммы 6 . В соответствии с (3.21) имеем $a_{s}^{l}=\varkappa^{2}\left\langle\nabla_{y} E_{l}^{T} A_{0} \nabla_{y} E_{s}\right\rangle_{\mathbf{o}}$ для $s, l=1,2$. Поэтому для $l=1,2$ вьполнено неравенство

$$
\left|\int_{0}^{t} \int_{\Omega_{l}^{\varepsilon}}\left(\left(E_{l}^{0} R_{l}^{0}\right)_{\varepsilon} * f+\left(E_{l}^{0} a_{s}^{l}\right)_{\varepsilon} v_{s}-\left(E_{l}^{0} M_{s}^{l}\right)_{\varepsilon} *\left(v_{s}^{\prime \prime}\right), r_{t}^{\prime}\right) d x d t\right| \leqslant C \sqrt{\varkappa}
$$

где учтены равенство (3.23), леммы 1,8 , неравенство (6.3) и гладкость $f, v_{1}$ и $v_{2}$. Следовательно, добавляя $C \sqrt{\varkappa}$ в правую часть неравенства $(6.2)$, интегралы из (6.4) для $l=1,2$ можно внести под знак модуля в (6.2).

Таким образом, доказано неравенство (4.36) с дополнительньм слагаемым $C \sqrt{\varkappa}$ в правой части. Поэтому, как и в $\S 4$ (см. (4.37)-(4.43)), получаем оценку

$$
\mathscr{E}(r) \leqslant C\left(\varepsilon\left(1+\varkappa^{2}+\varkappa\right)+\sigma+\sqrt{\varkappa}\right) .
$$


Переходя к первообразньм от решения задачи (1.4), как и в $\S 5$, можно избавиться от дополнительных предположений на исходные данные. При этом следует иметь в виду равенства (2.20). Поэтому из (6.5) получаем

$$
\begin{aligned}
& \left\|u-E_{s \varepsilon} v_{s}-P_{\varepsilon} * f+Q_{s \varepsilon} *\left(v_{s}^{\prime \prime}\right)\right\|_{C^{0}\left([0, T] ; L^{2}\left(\Omega_{0}^{\varepsilon}\right)^{n}\right)}^{2} \\
& \quad+\left\|u-E_{s \varepsilon} v_{s}\right\|_{C^{0}\left([0, T] ; L^{2}\left(\Omega_{12}^{\varepsilon}\right)^{n}\right)}^{2} \leqslant C\left(\varepsilon\left(1+\varkappa^{2}+\varkappa\right)+\sigma+\sqrt{\varkappa}\right),
\end{aligned}
$$

и утверждение теоремы 3 следует из лемм 6,7 и равенства (3.24).

ЗАмЕЧАНИЕ 6 . Пусть выполнены условия теоремы 3 и $w_{0}=0, w_{1}=0, f, g_{k} \in$ $C_{0}^{\infty}((0, T) \times \Omega)^{n}$, где $k=1, \ldots, n$. В этом случае можно просто повторить доказательство теоремы 4 с $\vartheta$, замененным на $\varkappa^{2}$. Тогда выполнено неравенство (4.43) - более точное, чем неравенство (6.5). Следовательно, используя (3.23), (3.24) и (6.3), получаем неравенство $(6.6) \mathrm{c} \sqrt{\varkappa}$, замененным на $\varkappa$.

\section{§7. Доказательство теоремы 4}

В случае, когда выполнено условие (1.23), “энергетическую” норму для задачи (1.4) следует определить равенством

$$
\begin{aligned}
\mathscr{E}_{\mu}(u) \equiv \mu\left\|u_{t}^{\prime}\right\|_{C^{0}\left([0, T] ; L^{2}\left(\Omega_{0}^{\varepsilon}\right)^{n}\right)}^{2}+\left\|u_{t}^{\prime}\right\|_{C^{0}\left([0, T] ; L^{2}\left(\Omega_{12}^{\varepsilon}\right)^{n}\right)}^{2} \\
+\sigma\|e(u)\|_{C^{0}\left([0, T] ; L^{2}\left(\Omega_{0}^{\varepsilon}\right)^{n \times n}\right)}^{2}+\|e(u)\|_{C^{0}\left([0, T] ; L^{2}\left(\Omega_{12}^{\varepsilon}\right)^{n \times n}\right)}^{2}
\end{aligned}
$$

Тогда с сохранением доказательства для $\mathscr{E}_{\mu}(u)$ выполнено неравенство леммы 1.

Пусть выполнены условия (1.1) и (1.23). Начальные члены асимптотитики решения задачи (1.4) можно определить равенством (3.49). Однако полезно подправить в этом разложении вектор-функцию $E_{2 \varepsilon} v_{2}$ на множестве $\Omega_{0}^{\varepsilon}$. Поэтому определим подправленную асимптотику $v_{a}$ равенством

$$
\begin{aligned}
& v_{a}=v+\frac{\varepsilon^{2}}{\sigma}\left(E_{2 \varepsilon} v_{2}\right.\left.+\varepsilon E_{2 \varepsilon}^{e} \nabla v_{2}\right)+\frac{\varepsilon^{2}}{\sigma}\left(p_{\varepsilon} f-q_{\varepsilon} v_{t t}^{\prime \prime}\right) \\
&+\varepsilon\left(N_{1 a}\right)_{\varepsilon} \eta \nabla v+\varepsilon\left(N_{1 b}\right)_{\varepsilon} g+\varepsilon\left(N_{2 a}^{1}\right)_{\varepsilon} \eta \nabla v+\varepsilon\left(N_{2 b}\right)_{\varepsilon} g,
\end{aligned}
$$

где $\eta$ определено в $\S 4, v$ является решением задачи (1.15) и $v_{2}$ удовлетворяет соотношениям (3.44) и (3.45). Кроме того, $p$ и $q$ определяются через решения задач (3.48) равенствами $p=\mu p^{0}, q=\mu q^{0}$ и $E_{2}^{e} \in H_{P}^{1}(Y)^{n \times \tilde{n} \times n}$ является 1-периодическим решением задачи Дирихле

$$
-\operatorname{div}_{y}\left(A_{0} \nabla_{y} E_{2}^{e}\right)=A_{0} \nabla_{y} E_{2} \quad \text { в } Y_{0}, \quad E_{2}^{e}=0 \text { на } \bar{Y}_{12} .
$$

Как и в $§ 5$, для периодических функций из (7.2) выполнены неравенства, аналогичные неравенствам (4.2). Кроме того, равенство (7.2) "почти" совпадает с равенством (5.1). Поэтому, заменяя $\mathscr{E}(r)$ на $\mathscr{E}_{\mu}(r)$ в $(5.4),(5.9),(5.17)$ и $(5.20)$, для доказательства теоремы 4 можно фактически повторить доказательство теоремы 1 из $\S 5$. При этом оценки соответствующих интегралов по $\Omega_{1}^{\varepsilon}$ и $\Omega_{2}^{\varepsilon}$ не изменяются, и следует только уточнить оценки интегралов по $\Omega_{0}^{\varepsilon}$. Оценим, например, вклад $J_{3}$ из (5.7) в неравенство (5.9). 
По определению $m_{0 \varepsilon}=\mu m_{\varepsilon}$ на $\Omega_{0}^{\varepsilon}$, где $m(y)$ удовлетворяет (1.9). Поэтому, используя неравенство Коши-Буняковского и $\delta$-неравенство, имеем

$$
\begin{aligned}
\left|\int_{0}^{t} \int_{\Omega_{0}^{\varepsilon}}\left(\varepsilon m_{0 \varepsilon} J_{3}, r_{t}^{\prime}\right) d x d t\right| & \leqslant \varepsilon \sqrt{\mu}\left|\int_{0}^{t} \int_{\Omega_{0}^{\varepsilon}}\left(m_{\varepsilon} J_{3}, \sqrt{\mu} r_{t}^{\prime}\right) d x d t\right| \\
& \leqslant \varepsilon \sqrt{\mu}\left(\frac{C}{2 \delta}+\delta C\left\|\sqrt{\mu} r_{t}^{\prime}\right\|_{C^{0}\left([0, T] ; L^{2}\left(\Omega_{0}^{\varepsilon}\right)^{n}\right)}^{2}\right) \\
& \leqslant C \varepsilon^{2} \mu+\delta_{4} \mathscr{E}_{\mu}(r),
\end{aligned}
$$

где $\delta=\delta_{4} /(C \varepsilon \sqrt{\mu})$. Таким образом, этот интеграл несущественен в (5.9). Аналогично оценивается последний интеграл в (5.16), поскольку $p=\mu p_{0}$ и $p_{0}$ удовлетворяет неравенству (4.2).

Определенные трудности возникают при оценке последнего интеграла в (5.15). Отметим, что $E_{2}^{e}$ выбрано так, что этот интеграл сокрашается с соответствующим интегралом от $\operatorname{div}_{y}\left(A_{0} \nabla_{y} E_{2}^{e}\right)$, но появляются дополнительные интегралы порядка $\varepsilon^{2}$. Точнее, в соответствии с (7.2) равенство (5.15) заменяется на равенство

$$
\begin{gathered}
-\varepsilon \int_{\Omega_{0}^{\varepsilon}}\left(\left(A_{0} \nabla_{y}\left(E_{2}+\varepsilon E_{2}^{e} \nabla_{x}\right)\right)_{\varepsilon} v_{2}, e\left(r_{t}^{\prime}\right)\right) d x=\varepsilon \int_{S_{0}^{\varepsilon}}\left(\left(\nu A_{0} \nabla_{y} E_{2}\right)_{\varepsilon} v_{2}, r_{t}^{\prime}\right) d s \\
+\varepsilon^{2} \int_{S_{0}^{\varepsilon}}\left(\left(\nu A_{0} \nabla_{y} E_{2}^{e}\right)_{\varepsilon} \nabla v_{2}, r_{t}^{\prime}\right) d s+\varepsilon^{2} \int_{\Omega_{0}^{\varepsilon}}\left(\left(A_{0} \nabla_{y} E_{2}^{e}\right)_{\varepsilon} \nabla \nabla v_{2}, r_{t}^{\prime}\right) d x,
\end{gathered}
$$

где использованы уравнения из (1.18) и (7.3).

В соответствии с (5.17)-(5.19) первый граничный интеграл из (7.5) сокрашается с суммой интегралов

$$
\int_{\Omega_{1}^{\varepsilon}}\left(\left(E_{1}^{0} \widehat{a}_{2}^{1}\right)_{\varepsilon} v_{2}, r_{t}^{\prime}\right) d x+\int_{\Omega_{2}^{\varepsilon}}\left(\left(E_{2}^{0} \widehat{a}_{2}^{2}\right)_{\varepsilon} v_{2}, r_{t}^{\prime}\right) d x
$$

из (5.17) с точностью до несушественных в (5.17) интегралов. Причем эти несущественные интегралы оцениваются на множествах $\Omega_{1}^{\varepsilon}$ и $\Omega_{2}^{\varepsilon}$ (см. (5.19)).

Аналогичньтм образом можно оценить второй граничный интеграл из (7.5). Действительно, введем обозначения

$$
b_{2}^{l}=\left\langle E_{l}^{T} A_{0} \nabla_{y} E_{2}\right\rangle_{\mathbf{o}}=-\left\langle E_{l}^{T} \operatorname{div}_{y}\left(A_{0} \nabla_{y} N_{2}^{e}\right)\right\rangle_{\mathbf{o}}=\int_{S_{l}} E_{l}^{T} \nu\left(A_{0} \nabla_{y} N_{2}^{e}\right) d s,
$$

где $l=1,2$ и использовано уравнение из (7.3) (см. (3.20) или (3.29)). Далее, рассмотрим сумму интегралов

$$
\varepsilon \int_{0}^{t} \int_{\Omega_{1}^{\varepsilon}}\left(\left(E_{1}^{0} b_{2}^{1}\right)_{\varepsilon} \nabla v_{2}, r_{t}^{\prime}\right) d x+\varepsilon \int_{0}^{t} \int_{\Omega_{2}^{\varepsilon}}\left(\left(E_{2}^{0} b_{2}^{2}\right)_{\varepsilon} \nabla v_{2}, r_{t}^{\prime}\right) d x .
$$

Модуль этой суммы оценивается величиной $\varepsilon C$ в силу леммы 1 , неравенств $(4.2)$ и гладкости $v_{2}$. Следовательно, сумму (7.8) можно внести под знак модуля в (5.17) (с сохранением этого неравенства) и сократить с вторьм граничным интегралом из (7.5) (с точностью до несущественных в (5.17) слагаемых). 
Таким образом, для доказательства теоремы 4 остается фактически оценить последний интеграл из (7.5). Обозначим этот интеграл через $I_{3}$. Тогда в соответствии с (4.5) получаем

$$
I_{3}=\varepsilon^{2} \int_{\Omega_{0}^{\varepsilon}}\left(\left(A_{0} \nabla_{y} E_{2}^{e}\right)_{\varepsilon} \nabla^{2} v_{2}, u_{t}^{\prime}\right) d x-\varepsilon^{2} \int_{\Omega_{0}^{\varepsilon}}\left(\left(A_{0} \nabla_{y} E_{2}^{e}\right)_{\varepsilon} \nabla^{2} v_{2},\left(v_{a}\right)_{t}^{\prime}\right) d x
$$

где последний интеграл несушественен в (5.17) в силу представления (7.2), неравенств (4.2) и гладкости $v, v_{2}$ и $g$.

Интегрируя по частям, как и в (2.6), получаем

$$
\begin{aligned}
& \left|\varepsilon^{2} \int_{0}^{t} \int_{\Omega_{0}^{\varepsilon}}\left(\left(A_{0} \nabla_{y} E_{2}^{e}\right)_{\varepsilon} \nabla^{2} v_{2}, u_{t}^{\prime}\right) d x d t\right| \\
& \leqslant\left|\varepsilon^{2} \int_{\Omega_{0}^{\varepsilon}}\left(\left(A_{0} \nabla_{y} E_{2}^{e}\right)_{\varepsilon} \nabla^{2} v_{2}, u\right) d x\right|+\left|\varepsilon^{2} \int_{0}^{t} \int_{\Omega_{0}^{\varepsilon}}\left(\left(A_{0} \nabla_{y} E_{2}^{e}\right)_{\varepsilon} \nabla^{2} v_{2}^{\prime}, u\right) d x d t\right| \\
& \quad+\left|\varepsilon^{2} \int_{\Omega_{0}^{\varepsilon}}\left(\left(A_{0} \nabla_{y} E_{2}^{e}\right)_{\varepsilon} \nabla^{2} v_{2}^{0}, w_{0}\right) d x\right|
\end{aligned}
$$

где $v_{2}^{0}=\left.v_{2}\right|_{t=0}$ и последний интеграл несущественен в $(5.17)$ в силу предположений на начальные данные.

Из неравенства леммы 1 следует, что $\|e(u)\|_{C^{0}\left([0, T] ; L^{2}(\Omega)^{n \times n}\right)} \leqslant C / \sqrt{\sigma}$ и $u=0$ на $\partial \Omega \times(0, T)$. Поэтому, учитьвая неравенство Корна и неравенство $Ф$ ридрихса, имеем для решения задачи (1.4) оценку $\|u\|_{C^{0}\left([0, T] ; L^{2}(\Omega)^{n}\right)} \leqslant C / \sqrt{\sigma}$ (см., например, [2], [3]). Используя эту оценку, неравенство Коши-Буняковского и гладкость $v_{2}$, можно оценить правую часть неравенства $(7.10)$ величиной $C \varepsilon(\varepsilon / \sqrt{\sigma}) \leqslant$ $C \varepsilon$, поскольку $\varepsilon / \sqrt{\sigma} \rightarrow 0$ при $\varepsilon \rightarrow 0$ и $\sigma \rightarrow 0$ в силу условия (1.1).

Таким образом, получена подходящая оценка для последнего интеграла из (5.15). Следовательно, как и в $\S 5$, имеем неравенство

$$
\mu\|u-v\|_{C^{0}\left([0, T] ; L^{2}\left(\Omega_{0}^{\varepsilon}\right)^{n}\right)}^{2}+\|u-v\|_{C^{0}\left([0, T] ; L^{2}\left(\Omega_{12}^{\varepsilon}\right)^{n}\right)}^{2} \leqslant C\left(\varepsilon+\sigma+\frac{\varepsilon^{2}}{\sigma}\right) .
$$

Пусть $v_{0}$ обозначает решение задачи $(1.15)$, в которой $M$ и $R$ заменены на $\widehat{M}, \widehat{R}$ соответственно. Тогда из неравенств (3.50) и (7.11) получаем

$$
\mu\left\|u-v_{0}\right\|_{C^{0}\left([0, T] ; L^{2}\left(\Omega_{0}^{\varepsilon}\right)^{n}\right)}^{2}+\left\|u-v_{0}\right\|_{C^{0}\left([0, T] ; L^{2}\left(\Omega_{12}^{\varepsilon}\right)^{n}\right)}^{2} \leqslant C\left(\varepsilon+\sigma+\mu^{2}+\frac{\varepsilon^{2}}{\sigma}\right)
$$

что завершает доказательство теоремы 4.

ЗАмЕчАНИЕ 7. В соответствии с неравенством (3.50) асимптотику по $\mu$ для $v$ из (7.11) можно уточнить и увеличить показатель степени $\mu$ в последнем неравенстве. Определяя дополнительные задачи на ячейке (типа задачи (7.3)), можно увеличить и показатели степеней $\sigma$ и $\varepsilon^{2} / \sigma$ в (7.11). Однако без дополнительных предположений не улучшается показатель степени $\varepsilon$ в (7.11), чему препятствует неулучшаемость оценки (4.6), связанная с краевьми эффектами в задаче (1.4) вблизи $2 \Omega$. 


\section{§ 8. Доказательство теоремы 5}

Пусть выполнены условия (1.2) и (1.23). Начальные члены асимптотического разложения решения задачи (1.4) можно определить равенством (3.51). Как и в $₹ 7$, подправим в этом разложении вектор-функции $E_{1 \varepsilon} v_{1}$ и $E_{2 \varepsilon} v_{2}$ на множестве $\Omega_{0}^{\varepsilon}$ и определим подправленную асимптотику $v_{a}$ равенством

$$
v_{a}=E_{s \varepsilon} v_{s}+\varepsilon E_{s \varepsilon}^{e} \nabla v_{s}+P_{\varepsilon} f-Q_{s \varepsilon} v_{s}^{\prime \prime}+\varepsilon\left(N_{s a}\right)_{\varepsilon} \eta \nabla v_{s}+\varepsilon\left(N_{s b}\right)_{\varepsilon} g_{s},
$$

где $\left(v_{1}, v_{2}\right)$ является решением задачи $(3.31), P=(\mu / \vartheta) p^{0}, Q_{s}=(\mu / \vartheta) q_{s}^{0}$ и использованы обозначения из $(3.51)$ и (4.1). Кроме того, $E_{2}^{e}$ является 1-периодическим решением задачи $(7.3)$ и $E_{1}^{e} \in H_{P}^{1}(Y)^{n \times n \times n}$ определяется как 1-периодическое решение задачи Дирихле

$$
-\operatorname{div}_{y}\left(A_{0} \nabla_{y} E_{1}^{e}\right)=A_{0} \nabla_{y} E_{1} \quad \text { в } Y_{0}, \quad E_{1}^{e}=0 \quad \text { на } \bar{Y}_{12}
$$

Напомним, что в (8.1) и далее предполагается суммирование по повторяющимся индексам $s=1,2$.

Как уже отмечалось в $\S 3$, симметричные матрицы $a_{0}=\vartheta \widetilde{a}$ и $\widetilde{m}$ из $(3.31),(3.32)$ неотрицательно определены. Поэтому сушествование и единственность достаточно гладкого решения задачи (3.31) проверяется по стандартной схеме (см. лемму 10 и [2], [3]). Однако при неоднородных начальных условиях в (3.31) константы в оценках соответствующих норм будут зависеть от $\vartheta$. Впрочем, несложно выписать явно эту зависимость, но мы не будем на этом останавливаться, поскольку в дальнейшем эта зависимость не используется (в отличие от неравенства теоремы 2, которое использовалось при доказательстве теоремы 3 ). Напомним только, что $\vartheta-$ постоянная из условия (1.2).

Как и в $\oint 5$, для периодических функций из (8.1) выполнены неравенства, аналогичные неравенствам (4.2), и без ограничения общности можно считать, что $w_{0}=0$ и $\left.g\right|_{t=0}=0$. Кроме того, представление (8.1) достаточно “близко" к представлению $(\overline{4} .1)$, в котором * будем рассматривать как обычное умножение. Поэтому, заменяя $\mathscr{E}(r)$ на $\mathscr{E} \mu(r)$ в соответствующих неравенствах из $\S 4$, будем придерживаться схемы доказательства теоремы 2, отмечая только существенные изменения. Из (3.6), (3.31), (4.2) и (8.1) следует, что

$$
\left\|\left.r_{t}^{\prime}\right|_{t=0}\right\|_{L^{2}(\Omega)^{n}}^{2}+\sigma\left\|e\left(\left.r\right|_{t=0}\right)\right\|_{L^{2}\left(\Omega_{0}^{\varepsilon}\right)^{n \times n}}^{2} \leqslant C\left(\varepsilon^{2}+\mu^{2}\right)
$$

и $e\left(\left.r\right|_{t=0}\right)=0$ на множестве $\bar{\Omega}_{12}^{\varepsilon}$ в силу предположений на начальные данные. Поэтому для доказательства теоремы 5 достаточно получить неравенство

$$
I \leqslant \frac{1}{2} \mathscr{E}_{\mu}(r)+C\left(\varepsilon+\sigma+\mu^{2}+\left|\vartheta-\frac{\sigma}{\varepsilon^{2}}\right|\right),
$$

где $I$ определено в (4.7) и постоянная $C$ не зависит от $\varepsilon, \sigma$ и $\mu$.

В соответствии с (8.1) равенства $(4.14)$ в рассматриваемом случае заменяются на равенства

$$
\begin{gathered}
\left(v_{a}\right)_{t t}^{\prime \prime}=E_{s \varepsilon} v_{s}^{\prime \prime}+\varepsilon J_{5}+\mu J_{6} \quad \text { в } \Omega_{0}^{\varepsilon} \\
\left(v_{a}\right)_{t t}^{\prime \prime}=E_{1 \varepsilon} v_{1}^{\prime \prime}+\varepsilon J_{5} \quad \text { в } \quad \Omega_{1}^{\varepsilon}, \quad\left(v_{a}\right)_{t t}^{\prime \prime}=E_{2 \varepsilon} v_{2}^{\prime \prime}+\varepsilon J_{5} \quad \text { в } \quad \Omega_{2}^{\varepsilon},
\end{gathered}
$$


где соответствующие интегралы с $J_{5}$ и $J_{6}$ несущественны в $(8.4)$ (см., например, (7.4)). Тогда сохраняются соотношения (4.15)-(4.17), но неравенство (4.18) заменяется на неравенство

$$
\begin{aligned}
I \leqslant \mid \int_{0}^{t} & {\left[\int_{\Omega_{0}^{\varepsilon}}\left(\left(r_{0}\right)_{\varepsilon} f, r_{t}^{\prime}\right) d x-\int_{\Omega_{0}^{\varepsilon}}\left(\left(m_{0} E_{s}\right)_{\varepsilon} v_{s}^{\prime \prime}, r_{t}^{\prime}\right) d x\right.} \\
& \left.+I_{1}+I_{2}-\int_{\Omega_{0}^{\varepsilon}}\left(A_{\varepsilon}^{\sigma} u^{2}, e\left(r_{t}^{\prime}\right)\right) d x\right] d t \mid \\
& +C\left(\varepsilon+\sigma+\mu^{2}\right)+\left(\delta_{1}+\delta_{2}+\delta_{4}\right) \mathscr{E}_{\mu}(r)
\end{aligned}
$$

где, например, $I_{2}$ определяется равенством (4.21).

Заменим равенство (4.24) на второе уравнение из (3.31). Тогда в соответствии с (4.19)-(4.28) получаем равенство

$$
I_{2}=\int_{\Omega_{2}^{\varepsilon}}\left(\left(E_{2}^{0} \widehat{m}_{s}^{2}\right)_{\varepsilon} v_{s}^{\prime \prime}+\left(E_{2}^{0} a_{s}^{2}\right)_{\varepsilon} v_{s}-\left(E_{2}^{0} \widehat{r}_{2}^{0}\right)_{\varepsilon} f, r_{t}^{\prime}\right) d x
$$

с точностью до несущественных в (8.4) слагаемых. Аналогичное (8.7) равенство вьполнено для интеграла $I_{1}$ из (8.6).

Рассмотрим теперь интеграл по $\Omega_{0}^{\varepsilon}$ из (8.6), содержащий $u^{2}=u^{1}+u^{0}$. Для этого интеграла выполнены соотношения (4.30), в которых $\omega=\sigma / \varepsilon^{2}-\vartheta$ и последний интеграл несушественен в (8.4) (см. (4.31)). Здесь учтено, что величина $\varepsilon / \sqrt{\sigma}$ стремится к $1 / \sqrt{\vartheta}$ и поэтому ограничена при $\varepsilon \rightarrow 0$ и $\sigma \rightarrow 0$. Аналогично вьполнены соотношения (4.32), в которых последние два интеграла несушественны в (8.4) (см. (4.33)).

Кроме того, выполнены соотношения (4.34), в которых предпоследний интеграл равен нулю, а для оценки последнего интеграла следует иметь в виду второе слагаемое из $(8.1)$, подправляющее $E_{s \varepsilon} v_{s}$ на множестве $\Omega_{0}^{\varepsilon}$. Тогда в соответствии с (7.5)-(7.10) вклад последнего интеграла из (4.34) в неравенство (8.6) оценивается величиной $C \varepsilon$. Аналогично выполнены соотношения (4.35), в которых предпоследний интеграл сокрашается с (первым) интегралом по $\Omega_{0}^{\varepsilon}$ из (8.6), содержащим $f$ (в силу (3.48)), а вклад последнего интеграла в неравенство (8.6) оценивается величиной $C \mu^{2} \varepsilon$ (см. (7.4)). Напомним, что $P=(\mu / \vartheta) p^{0}$ в (8.1). Аналогичные (4.35) равенства выполнены для интеграла по $\Omega_{0}^{\varepsilon}$ из $(8.6)$, содержащего $q_{s}$ для $s=1,2$.

Таким образом, доказано неравенство (4.36), в котором последняя строка заменена правой частью из (8.4) и интегралы по $\Omega_{1}^{\varepsilon}$ и $\Omega_{2}^{\varepsilon}$ заменены на $I_{1}$ и $I_{2}$. Для $s, l=1,2$ постоянные матрицы $\widehat{m}_{s}^{l}, a_{s}^{l}$ и $\widehat{r}_{s}^{0}$ (см. (8.7)) определены так, что интегралы $I_{1}$ и $I_{2}$ сокращаются с граничными интегралами из (4.36) в соответствии с $(3.21),(3.29),(3.30)$ и $(5.17)-(5.19)$. Поэтому, как и в $\S 5$, имеем

$$
\begin{aligned}
\mu\left\|u-E_{s \varepsilon} v_{s}\right\|_{C^{0}\left([0, T] ; L^{2}\left(\Omega_{0}^{\varepsilon}\right)^{n}\right)}^{2}+\left\|u-E_{s \varepsilon} v_{s}\right\|_{C^{0}\left([0, T] ; L^{2}\left(\Omega_{12}^{\varepsilon}\right)^{n}\right)}^{2} & \leqslant C\left(\varepsilon+\sigma+\mu^{2}+\left|\vartheta-\frac{\sigma}{\varepsilon^{2}}\right|\right) .
\end{aligned}
$$

Пусть $\left(v_{1}^{0}, v_{2}^{0}\right)$ обозначает решение задачи $(1.21)$, в которой $M_{s}^{l}=0$ и $R_{s}^{l}=0$ для $s, l=1,2$. Эта задача определяет первое приближение по $\mu$ для задачи (3.31), поскольку выполнено условие (1.23). Поэтому в последнем неравенстве можно заменить $\left(v_{1}, v_{2}\right)$ на $\left(v_{1}^{0}, v_{2}^{0}\right)$, что завершает доказательство теоремы 5 . 


\section{§9. Доказательство теоремы 6}

Пусть выполнены условия (1.3), (1.23) и (1.25). В соответствии с (3.52) определим подправленную асимптотику $v_{a}$ равенством

$$
v_{a}=E_{s \varepsilon} v_{s}+P_{\varepsilon} f-Q_{s \varepsilon} v_{s}^{\prime \prime}+\varepsilon\left(N_{s a}\right)_{\varepsilon} \eta \nabla v_{s}+\varepsilon\left(N_{s b}\right)_{\varepsilon} g_{s}
$$

где $P=\left(\varepsilon^{2} \mu / \sigma\right) p^{0}, Q_{s}=\left(\varepsilon^{2} \mu / \sigma\right) q_{s}^{0}$ и $\left(v_{1}, v_{2}\right)$ - решение задачи (3.31), в которой $a_{s}^{l}=0$ для $s, l=1,2 . \mathrm{B}(9.1)$ использованы обозначения из (3.52) и (4.1).

Как и в $\S 8$, достаточно гладкое решение рассматриваемой задачи (3.31) сушествует и определено однозначно. Для периодических функций из (9.1) выполнены неравенства, аналогичные неравенствам (4.2), и без ограничения общности можно считать, что $w_{0}=0$ и $\left.g\right|_{t=0}=0$. Кроме того, представление (9.1) достаточно “близко" к представлению (8.1). Поэтому, как и в $\S 8$, будем придерживаться схемы доказательства теоремы 2.

Из (3.6), (3.31), (4.2) и представления (9.1) следует, что

$$
\left\|\left.r_{t}^{\prime}\right|_{t=0}\right\|_{L^{2}(\Omega)^{n}}^{2}+\sigma\left\|e\left(\left.r\right|_{t=0}\right)\right\|_{L^{2}\left(\Omega_{0}^{\varepsilon}\right)^{n \times n}}^{2} \leqslant C\left(\varepsilon^{2}+\mu^{2}+\left(\frac{\varepsilon^{2} \mu}{\sigma}\right)^{2}\right)
$$

и $e\left(\left.r\right|_{t=0}\right)=0$ на множестве $\bar{\Omega}_{12}^{\varepsilon}$ в силу предположений на начальные данные. Поэтому для доказательства теоремы 6 достаточно получить неравенство

$$
I \leqslant \frac{1}{2} \mathscr{E}_{\mu}(r)+C\left(\varepsilon+\sigma+\mu^{2}+\frac{\sigma}{\varepsilon^{2}}+\left(\frac{\varepsilon^{2} \mu}{\sigma}\right)^{2}\right)
$$

где $I$ определено в (4.7) и постоянная $C$ не зависит от $\varepsilon, \sigma$ и $\mu$.

В соответствии с (9.1) выполнены равенства (8.5), в которых $\mu J_{6}$ следует заменить на $\left(\varepsilon^{2} \mu / \sigma\right) J_{6}$. Тогда соответствующие интегралы с $J_{5}$ и $J_{6}$ несушественны в (9.3) (см. (7.4)). Кроме того, сохраняются соотношения (4.15)-(4.17), но неравенство (4.18) заменяется на неравенство

$$
\begin{aligned}
I \leqslant \mid \int_{0}^{t} & {\left[\int_{\Omega_{0}^{\varepsilon}}\left(\left(r_{0}\right)_{\varepsilon} f, r_{t}^{\prime}\right) d x-\int_{\Omega_{0}^{\varepsilon}}\left(\left(m_{0} E_{s}\right)_{\varepsilon} v_{s}^{\prime \prime}, r_{t}^{\prime}\right) d x\right.} \\
& \left.+I_{1}+I_{2}-\int_{\Omega_{0}^{\varepsilon}}\left(A_{\varepsilon}^{\sigma} u^{2}, e\left(r_{t}^{\prime}\right)\right) d x\right] d t \mid \\
& +C\left(\varepsilon+\sigma+\mu^{2}+\frac{\sigma}{\varepsilon^{2}}\right)+\left(\delta_{1}+\delta_{2}+\delta_{4}\right) \mathscr{E}_{\mu}(r),
\end{aligned}
$$

где, например, $I_{2}$ определяется равенством (8.7), в котором $a_{s}^{2}=0$ для $s=1,2$ (см. (4.19)-(4.28)). Аналогичное (8.7) равенство выполнено для $I_{1}$ из (9.4).

Рассмотрим теперь интеграл по $\Omega_{0}^{\varepsilon}$ из $(9.4)$, содержаший $u^{2}=u^{1}+u^{0}$. Непосредственно из $(1.6),(4.15)$ и (9.1) следует, что

$$
\int_{0}^{t} \int_{\Omega_{0}^{\varepsilon}}\left(A_{\varepsilon}^{\sigma} u^{1}, e\left(r_{t}^{\prime}\right)\right) d x d t=\int_{0}^{t} \int_{\Omega_{0}^{\varepsilon}}\left(\frac{\sqrt{\sigma}}{\varepsilon}\left(A_{0} \nabla_{y} E_{s}\right)_{\varepsilon} v_{s}, \sqrt{\sigma} e\left(r_{t}^{\prime}\right)\right) d x d t
$$


(см. (4.30)). Используя неравенство Коши-Буняковского, $\delta$-неравенство и интегрируя по частям в $(9.5)$, как и в $(4.17)$, получаем

$$
\left|\int_{0}^{t} \int_{\Omega_{0}^{\varepsilon}}\left(A_{\varepsilon}^{\sigma} u^{1}, e\left(r_{t}^{\prime}\right)\right) d x d t\right| \leqslant C \frac{\sigma}{\varepsilon^{2}}+\delta_{5} \mathscr{E}_{\mu}(r) .
$$

Следовательно, интеграл (9.5) несущественен в (9.4).

Непосредственно из (1.6), (4.15) и (9.1) также имеем

$$
\begin{aligned}
\int_{\Omega_{0}^{\varepsilon}}\left(A_{\varepsilon}^{\sigma} u^{0}, e\left(r_{t}^{\prime}\right)\right) d x=\varepsilon \mu \int_{\Omega_{0}^{\varepsilon}}\left(\left(A_{0} \nabla_{y} p^{0}\right)_{\varepsilon} f, e\left(r_{t}^{\prime}\right)\right) d x & \\
& -\varepsilon \mu \int_{\Omega_{0}^{\varepsilon}}\left(\left(A_{0} \nabla_{y} q_{s}^{0}\right)_{\varepsilon} v_{s}^{\prime \prime}, e\left(r_{t}^{\prime}\right)\right) d x .
\end{aligned}
$$

Следовательно, как и в (4.35), получаем

$$
\begin{aligned}
& -\varepsilon \mu \int_{\Omega_{0}^{\varepsilon}}\left(\left(A_{0} \nabla_{y} p^{0}\right)_{\varepsilon} f, e\left(r_{t}^{\prime}\right)\right) d x=\varepsilon \mu \int_{S_{0}^{\varepsilon}}\left(\left(\nu A_{0} \nabla_{y} p^{0}\right)_{\varepsilon} f, r_{t}^{\prime}\right) d s \\
& \quad+\mu \int_{\Omega_{0}^{\varepsilon}}\left(\left(\operatorname{div}_{y} A_{0} \nabla_{y} p^{0}\right)_{\varepsilon} f, r_{t}^{\prime}\right) d x+\varepsilon \mu \int_{\Omega_{0}^{\varepsilon}}\left(\left(A_{0} \nabla_{y} p^{0}\right)_{\varepsilon} \nabla f, r_{t}^{\prime}\right) d x,
\end{aligned}
$$

где предпоследний интеграл сокрашается с (первым) интегралом по $\Omega_{0}^{\varepsilon}$ из (9.4), содержащим $f$ (в силу (3.48)), а вклад последнего интеграла в неравенство (9.4) оценивается величиной $\varepsilon^{2} \mu C$ (см. (7.4)). Аналогичные (9.8) равенства выполнены для интеграла по $\Omega_{0}^{\varepsilon}$ из $(9.7)$, содержашего $q_{s}$ для $s=1,2$.

Таким образом, доказано аналогичное (4.36) неравенство

$$
\begin{gathered}
I \leqslant\left|\int_{0}^{t}\left[\varepsilon \mu \int_{S_{0}^{\varepsilon}}\left(\left(\nu A_{0} \nabla_{y} p^{0}\right)_{\varepsilon} f, r_{t}^{\prime}\right) d s-\varepsilon \mu \int_{S_{0}^{\varepsilon}}\left(\left(\nu A_{0} \nabla_{y} q_{s}^{0}\right)_{\varepsilon} v_{s}^{\prime \prime}, r_{t}^{\prime}\right) d s+I_{1}+I_{2}\right] d t\right| \\
+C\left(\varepsilon+\sigma+\mu^{2}+\frac{\sigma}{\varepsilon^{2}}\right)+\left(\delta_{1}+\delta_{2}+\delta_{4}+\delta_{5}\right) \mathscr{E}_{\mu}(r) .
\end{gathered}
$$

Поэтому доказательство теоремы 6 завершается, как и доказательство теоремы 5 из предыдушего параграфа.

\section{§10. Доказательство теорем 7 и 8}

Пусть выполнены условия (1.3), (1.23) и (1.26). Определим $P=P^{0}$ и $Q_{s}=Q_{s}^{0}$ для $s=1,2$. Здесь, как и в п. $7 \S 3$, матричнозначные функции $P^{0}, Q_{1}^{0}$ и $Q_{2}^{0}$ являются 1-периодическими решениями задач (1.19), в которых $m_{0}, r_{0}$ и $\vartheta$ заменены на $m, r$ и $\theta$ соответственно. Тогда выполнены неравенство (4.3) и аналогичные неравенства для $Q_{1}$ и $Q_{2}$ с $\vartheta$, замененньм на $\theta$. В этих неравенствах и далее в этом параграфе постоянная $C$ не зависит от $\varepsilon, \sigma, \mu$ и $\theta$. Вычислим по этим $P, Q_{1}$ и $Q_{2}$ коэффициенты из равенств (1.20), где $m_{0}=\mu m$ и $r_{0}=\mu r$ в соответствии с условием (1.23). Пусть $\left(v_{1}, v_{2}\right)$ является решением задачи (1.21), в которой $a_{s}^{l}=0$ для $s, l=1,2$. Сушествование и единственность достаточно гладкого решения этой задачи проверяется, как и в лемме 10. Причем, константы в оценках соответствуюших норм этого решения не зависят от $\mu$ (см. доказательство леммы 10$)$. 
Учитьвая (3.54), определим подправленную асимптотику $v_{a}$ равенством (4.1). Тогда, заменяя $\mathscr{E}(r)$ на $\mathscr{E}_{\mu}(r)$ в соответствующих формулах из $\S 4$, можно фактически повторить доказательство теоремы 2. Разумеется, в равенствах (4.25) и (4.29) следует положить $a_{s}^{l}=0$ для $s, l=1,2$. Далее, для интеграла по $\Omega_{0}^{\varepsilon}$ из (4.8), содержащего $u^{1}=u^{2}-u^{0}$, выполнено неравенство (9.6). Следовательно, этот интеграл несущественен в соответствуюшем неравенстве. Кроме того, в рассматриваемом случае не нужны соотношения (4.30), (4.31) и (4.34), поскольку именно для оценки граничного интеграла из (4.34) использовались ненулевые матрицы $a_{s}^{l}$ из (1.20) (см., например, (5.17)-(5.19)).

Таким образом, в соответствии с доказательством теоремы 2 имеем

$$
\mathscr{E}_{\mu}(r) \leqslant C\left(\varepsilon(1+\sqrt{\theta})+\sigma+\frac{\sigma}{\varepsilon^{2}}+\sqrt{\mu} \frac{\varepsilon \sqrt{\mu}}{\sqrt{\sigma \theta}}|v|\right),
$$

где $v=\sigma /\left(\varepsilon^{2} \mu\right)-\theta$. Отметим, что величина $\varepsilon \sqrt{\mu} / \sqrt{\sigma}$ стремится к $1 / \sqrt{\theta}$ и поэтому ограничена при $\varepsilon \rightarrow 0, \sigma \rightarrow 0$ и $\mu \rightarrow 0$ в силу условия (1.26).

Пусть $\left(v_{1}^{0}, v_{2}^{0}\right)$ обозначает решение задачи $(1.22)$. Из леммы $7,(1.23),(3.23)$ и (4.3) следует, что эта задача определяет первое приближение по $\mu$ для задачи (1.21), в которой $a_{l}^{s}=0$ для $s, l=1,2$. Поэтому из леммы 7 и (10.1) получаем

$$
\begin{array}{r}
\mu\left\|u-E_{s \varepsilon} v_{s}^{0}-P_{\varepsilon} * f+Q_{s \varepsilon} *\left(v_{s}^{0}\right)_{t t}^{\prime \prime}\right\|_{C^{0}\left([0, T] ; L^{2}\left(\Omega_{0}^{\varepsilon}\right)^{n}\right)}^{2}+\left\|u-E_{s \varepsilon} v_{s}^{0}\right\|_{C^{0}\left([0, T] ; L^{2}\left(\Omega_{12}^{\varepsilon}\right)^{n}\right)}^{2} \\
\leqslant C\left(\varepsilon(1+\sqrt{\theta})+\sigma+\mu^{2}+\frac{\sigma}{\varepsilon^{2}}+\sqrt{\mu} \frac{\varepsilon \sqrt{\mu}}{\sqrt{\sigma \theta}}|v|\right),
\end{array}
$$

что завершает доказательство теоремы 7 .

Наконец, доказательство теоремы 8 повторяет доказательство теоремы 7 с соответствующими изменениями, описанньпи в $\S 6$.

В заключение отметим, что в условиях замечаний 3 и 5 для доказательства теорем 1-8 достаточно фактически повторить доказательства из $\S \S 4-10$, в которых 1 и 2 заменены на $l_{1}=1, \ldots, s_{1}$ и $l_{2}=s_{1}+1, \ldots, s_{1}+s_{2}$ соответственно.

\section{Список литературы}

1. Жиков В. В. Связность и усреднение. Примеры фрактальной проводимости // Матем. сб. 1996. Т. 187. № 8. С. 3-40.

2. Лионс Ж.-Л., Мадженес Э. Неоднородные граничные задачи и их приложения. М.: Мир, 1971.

3. Дюво Г., Лионс Ж.-Л. Неравенства в механике и физике. М.: Наука, 1980.

4. Бахвалов Н. С., Панасенко Г. П. Осреднение процессов в периодических средах. М.: Наука, 1984.

5. Жиков В.В., Козлов С. М., Олейник О.А. Усреднение дифференциальных операторов. М.: Физматлит, 1993.

6. Лады женская O. А. Смешанная задача для гиперболического уравнения. М.: ГИТТЛ, 1953.

7. Сандраков Г. В. Осреднение линеаризованной системы гидродинамики с малой вязкостью и скорость звука в смесях // Препринт ОВМ АН СССР. № 178. М.: ОВМ АН СССР, 1987.

8. Сандраков Г.В. Принципы осреднения уравнений с быстроосциллирующими коэффициентами // Матем. сб. 1989. Т. 180. № 12. С. 1634-1679.

9. Панасенко Г. П. Многокомпонентное осреднение процессов в сильно неоднородных структурах // Матем. сб. 1990. Т. 181. №1. С. 134-142. 
10. Панасенко Г. П. Численно-асимптотический метод многокомпонентного осреднения для уравнений с контрастными коэффициентами // ЖВМ и МФ. 1990. Т. 30. № 2. С. 243-253.

11. Бахвалов Н. C., Эглит M. Э. О предельном поведении периодических сред с мягкомодулњными включениями // ЖВМ и МФ. 1995. Т. 35. №6. С. 905-917.

12. Сандраков Г. В. Осреднение нестационарных уравнений с контрастньпи коэффициентами // Докл. РАН. 1997. Т. 355. № 5. С. 605-608.

13. Сандраков Г. В. Осреднение нестационарных задач теории сильно неоднородных упругих сред // Докл. РАН. 1998. Т. 358. №3. С. 309-311.

14. Темам Р. Уравнения Навье-Стокса. М.: Мир, 1981.

15. Владимиров В. С. Уравнения математической физики. М.: Наука, 1988.

16. Агранович M. С., Вишик М. И. Эллиптические задачи с параметром и параболические задачи общего вида // УМН. 1964. Т. 19. № 3,. С. 53-161.

17. Хилле Э., Филлипс Р. Функциональный анализ и полугруппы. М.: ИЛ, 1962.

Московский государственный

Поступила в редакцию

университет им. М.В. Ломоносова

24.03.1999

E-mail: sandrako@compmath.math.msu.su 